\title{
Oxidative Coupling of Arylboronic Acids with Arenes via Rh-Catalyzed Direct C-H Arylation
}

\author{
Thomas Vogler and Armido Studer* \\ Fachbereich Chemie, Organisch-Chemisches Institut, Westfälische Wilhelms-Universität, \\ Corrensstrasse 40, 48149 Münster \\ studer@uni-muenster.de
}

\section{Supporting Information}

\section{General}

All reactions involving air- or moisture-sensitive reagents or intermediates were carried out in heat-gun-dried glassware under an argon atmosphere and were performed using standard Schlenk techniques. All solvents for filtration and flash chromatography were distilled before use. DME was distilled from sodium under argon, toluene was distilled from $\mathrm{Na}$ under argon. 1,4-Dioxane (Acros, extra dry, water $<50 \mathrm{ppm}$ ) was used without further purification, tert-butanol (Fluka, $>99.7 \%$ ) was used as received.

${ }^{1} \mathrm{H}-\mathrm{NMR}(300 \mathrm{MHz})$ and ${ }^{13} \mathrm{C}-\mathrm{NMR}(75 \mathrm{MHz})$ spectra were recorded on a Bruker DPX 300 or a Varian 600 unity plus spectrometer. Chemical shifts $\delta$ in ppm are referenced to the solvent residual peak as an internal standard. TLC was carried out on Merck silica gel $60 \mathrm{~F}_{254}$ plates; detection by $\mathrm{UV}$ or dipping into a soln. of $\mathrm{Ce}\left(\mathrm{SO}_{4}\right)_{2} \cdot \mathrm{H}_{2} \mathrm{O}(10 \mathrm{~g})$, phosphormolybdic acid hydrate $(25 \mathrm{~g})$, conc. $\mathrm{H}_{2} \mathrm{SO}_{4}(60 \mathrm{~mL})$ and $\mathrm{H}_{2} \mathrm{O}(0.94 \mathrm{~L})$ or $\mathrm{NaHCO}_{3}(5.0 \mathrm{~g}), \mathrm{KMnO}_{4}(1.5 \mathrm{~g})$ and $\mathrm{H}_{2} \mathrm{O}(0.40 \mathrm{~L})$ followed by heating. Flash chromatography (FC) was carried out on Merck or Fluka silica gel 60 (40-63 $\mu \mathrm{m})$ with an argon pressure of about 0.1-0.5 bar. GC spectra were acquired on a Hewlett-Packard HP 6890 Series GC system equipped with a HP 5 column $(30 \mathrm{~m} \times 0.32 \mathrm{~mm}$, film thickness $0.25 \mu \mathrm{m})$ or a HP 1 column $(25 \mathrm{~m} \times 0.32 \mathrm{~mm}$, film thickness $0.25 \mu \mathrm{m})$ using hydrogen as carrier gas. IR spectra were recorded on a Digilab FTS 4000 equipped with a MKII Golden Gate Single Reflection ATR System. Melting points were determined with a Stuart SMP10 and are uncorrected. GC-MS (EI) was performed on a Waters-Micromass GC TOF. ESI-MS and HRMS were performed using a Bruker MicroTof. 
Following chemicals were purchased and used as received:

3-Acetylphenylboronic acid (ABCR, 97\%), aniline (Merck, >99\%), 1,4-bis(diphenylphosphino)butane (Alfa Aesar, 98\%), 3,5-bis(trifluoromethyl)benzeneboronic acid (Alfa Aesar, 97+\%), 2-bromopyridine (Merck, 98\%), 3-chlorobenzeneboronic acid (Alfa Aesar, 97\%), chlorobis(cyclooctene)rhodium(I) dimer (Acros, 98\%), chloro(1,5-cyclooctadiene)rhodium(I) dimer (ABCR, 98\%), $\mu$-dichlorotetraethylene dirhodium(I) (Acros), 2-ethoxyphenylboronic acid (Acros), 4-fluorophenylboronic acid (ABCR, 98\%), hydroxy(1,5-cyclooctadiene)rhodium(I) dimer (ABCR, 97\%), 2-methoxybenzaldehyde (Fluka, >98\%), 4-methoxyphenylboronic acid (ABCR, 97\%), 3,4-methylenedioxybenzeneboronic acid (Alfa Aesar, 98\%), 2-methylphenylboronic acid (Aldrich, >95\%), 4-methylphenylboronic acid (ABCR, 97\%), phenylboronic acid (ABCR, 98\%), 2-phenylpyridine (Aldrich, 98\%), trans-2-phenylvinylboronic acid (Aldrich, 97\%), tetrakis(triphenylphosphine) palladium(0) (Aldrich, 99\%), 2,2,6,6-tetramethylpiperidin- $N$-oxyl radical (TEMPO, CIBA Specialty Chemicals), 2-(2-thienyl)pyridine (ABCR, 97\%), tris(4-methoxyphenyl)phosphine (Alfa Aesar, 98\%), tris(4-trifluoromethylphenyl) phosphine (Alfa Aesar, 98\%), 4-vinylbenzeneboronic acid (Alfa Aesar, 98\%). 


\section{Preparation of Starting Materials}

\section{2-(2-Ethoxyphenyl)pyridine (3)}

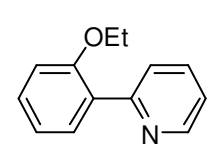

To a solution of 2-bromopyridine $(0.29 \mathrm{~mL}, 3.0 \mathrm{mmol})$ in DME $(9.0 \mathrm{~mL})$ was added tetrakis(triphenylphosphine)palladium(0) (104 mg, $90.0 \mu \mathrm{mol})$ and the mixture was stirred at $\mathrm{rt}$ for $10 \mathrm{~min}$. 2-Ethoxyphenylboronic acid $(0.55 \mathrm{~g}, 3.3 \mathrm{mmol})$ and a solution of sodium hydrogen carbonate $(0.76 \mathrm{~g}, 9.0 \mathrm{mmol}$ in 9.0 $\mathrm{mL}$ of water) were added and the mixture refluxed for $7 \mathrm{~h}$. Water $(10 \mathrm{~mL})$ was added and the mixture was extracted with MTBE $(3 \times 20 \mathrm{~mL})$. The combined organic layers were dried over $\mathrm{MgSO}_{4}$ and the volatiles were removed under vacuum. The crude product was purified by FC (pentane/MTBE 10:1) and 3 was obtained as pale yellow oil (0.52 $\mathrm{g}$, $2.6 \mathrm{mmol}, 87 \%$ ). IR (neat): 3063m, 2982m, 2878m, 1582s, 1494s, 1451s, 1423s, 1303m, $1232 s, 1157 m, 1126 s, 1041 s, 922 m, 791 m, 750 s .{ }^{1} \mathrm{H}-\mathrm{NMR}\left(300 \mathrm{MHz}, \mathrm{CDCl}_{3}\right): \delta=8.61$ $(b r, 1 \mathrm{H}, \mathrm{CH}) ; 7.86$ - $7.76(m, 1 \mathrm{H}, \mathrm{CH}) ; 7.75$ - $7.67(m, 1 \mathrm{H}, \mathrm{CH}) ; 7.67$ - $7.59(m, 1 \mathrm{H}$, $\mathrm{CH}) ; 7.32$ - 7.19 (m, $1 \mathrm{H}, \mathrm{CH}) ; 7.17$ - 7.08 (m, $1 \mathrm{H}, \mathrm{CH}) ; 7.03$ - 6.92 (m, $1 \mathrm{H}, \mathrm{CH}) ; 6.92$ $6.83(m, 1 \mathrm{H}, \mathrm{CH}) ; 4.05-3.92\left(m, 2 \mathrm{H}, \mathrm{CH}_{2}\right) ; 1.33-1.21\left(m, 3 \mathrm{H}, \mathrm{CH}_{3}\right) .{ }^{13} \mathrm{C}-\mathrm{NMR}$ $\left(75 \mathrm{MHz}, \mathrm{CDCl}_{3}\right): \delta=156.4(\mathrm{C}), 155.6(\mathrm{C}), 148.5(\mathrm{CH}), 136.3(\mathrm{CH}), 131.2(\mathrm{CH}), 130.3$ $(\mathrm{CH}), 128.1(\mathrm{C}), 125.4(\mathrm{CH}), 121.7(\mathrm{CH}), 121.1(\mathrm{CH}), 112.6(\mathrm{CH}), 64.2\left(\mathrm{CH}_{2}\right), 14.8$ $\left(\mathrm{CH}_{3}\right) . \mathrm{MS}(\mathrm{EI}) \mathrm{m} / z: 199$ (22, [M] $\left.]^{+}\right), 184$ (100), 170 (33), 155 (60), 141 (14), 127 (8), 117 (20), 80 (15). HRMS (ESI): calcd for $[\mathrm{M}+\mathrm{H}]^{+}:$200.1070; found: 200.1074.

\section{$N$-(2-Methoxybenzylidene)aniline (8)}

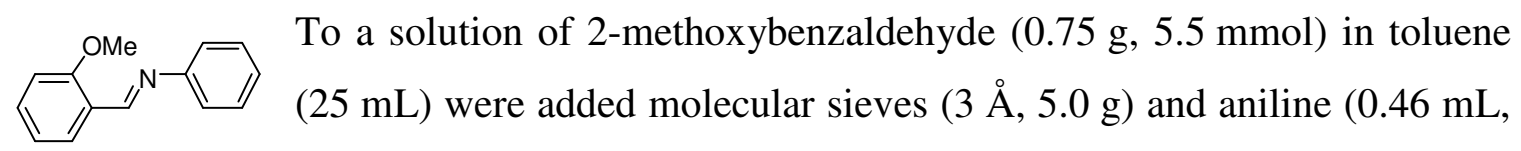
$5.0 \mathrm{mmol}){ }^{1}$ The reaction mixture was stirred at $\mathrm{rt}$ for $20 \mathrm{~h}$ and filtered over celite. Removal of the volatiles under vacuum yielded 8 as yellow solid $(0.99 \mathrm{~g}, 4.7 \mathrm{mmol}$, $94 \%)$.

${ }^{1} \mathrm{H}-\mathrm{NMR}\left(300 \mathrm{MHz}, \mathrm{CDCl}_{3}\right): \delta=8.92(b r, 1 \mathrm{H}, \mathrm{CH}) ; 8.20-8.12(m, 1 \mathrm{H}, \mathrm{CH}) ; 7.49$ 7.34 (m, 3 H, CH); 7.25 - 7.17 (m, 3 H, CH); 7.11 - $7.00(m, 1 \mathrm{H}, \mathrm{CH}) ; 7.00$ - 6.93 (m, $1 \mathrm{H}, \mathrm{CH}) ; 3.90\left(s, 3 \mathrm{H}, \mathrm{CH}_{3}\right)$. The spectroscopic data obtained are in accordance with those described in the literature. ${ }^{2}$ 


\section{General procedure for the Rhodium-Catalyzed C-H Arylation using a pyridyl- directing group (GP1)}

$\mu$-Dichlorotetraethylene dirhodium(I) $\quad(4.9 \mathrm{mg}, \quad 13 \mu \mathrm{mol}) \quad$ and tris(4-trifluoromethylphenyl)phosphine $(23.3 \mathrm{mg}, 50.0 \mu \mathrm{mol})$ in dioxane $(0.5 \mathrm{~mL})$ were heated in a reaction tube at $90{ }^{\circ} \mathrm{C}$ for $5 \mathrm{~min}$. The mixture was cooled to room temperature. The corresponding boronic acid $(1.00 \mathrm{mmol})$, TEMPO $(156 \mathrm{mg}, 1.00 \mathrm{mmol})$, the pyridine derivative $(0.25 \mathrm{mmol}), t$ - $\mathrm{BuOH}(0.1 \mathrm{~mL})$ and dioxane $(0.5 \mathrm{~mL})$ were added. The reaction mixture was stirred in a sealed tube at $130{ }^{\circ} \mathrm{C}$ for $9 \mathrm{~h}$ and filtered through a plug of silica gel ( $c a .40 \mathrm{~mL}$ MTBE as eluent). The filtrate was concentrated under vacuum, and the residue was purified by FC.

\section{General procedure for the Rhodium-Catalyzed C-H Arylation using an imine as directing group (GP2)}

$\mu$-Dichlorotetraethylene dirhodium(I) $\quad(4.9 \mathrm{mg}, \quad 13 \mu \mathrm{mol})$ and tris(4-trifluoromethylphenyl)phosphine $(23.3 \mathrm{mg}, 50.0 \mu \mathrm{mol})$ in dioxane $(0.5 \mathrm{~mL})$ were heated in a reaction tube at $90{ }^{\circ} \mathrm{C}$ for $5 \mathrm{~min}$. The mixture was cooled to room temperature. The corresponding boronic acid $(1.50 \mathrm{mmol})$, TEMPO $(234 \mathrm{mg}, \quad 1.50 \mathrm{mmol})$, $N$-(2-methoxybenzylidene)aniline (8) $(53 \mathrm{mg}, 0.25 \mathrm{mmol}), t$ - $\mathrm{BuOH}(0.1 \mathrm{~mL})$ and dioxane $(0.5 \mathrm{~mL})$ were added. The reaction mixture was stirred in a sealed tube at $90{ }^{\circ} \mathrm{C}$ for $22 \mathrm{~h}$ and filtered through a plug of basic alumina ( $c a .40 \mathrm{~mL}$ acetone as eluent). To the filtrate were added $\mathrm{H}_{2} \mathrm{O}(5.0 \mathrm{~mL})$ and aq. $\mathrm{HCl}(1.0 \mathrm{M}, 5.0 \mathrm{~mL})$ and the reaction mixture stirred for $30 \mathrm{~min}$ at $\mathrm{rt}$. The mixture was extracted with MTBE $(3 \times 20 \mathrm{~mL})$. The combined organic layers were washed with aq. sat. $\mathrm{NaHCO}_{3}(20 \mathrm{~mL})$ and dried over $\mathrm{MgSO}_{4}$. The filtrate was concentrated under vacuum, and the residue was purified by FC. 


\section{Rhodium-Catalyzed C-H Arylation using Oxygen or Air as (Co-)Oxidant (GP3)}

$\mu$-Dichlorotetraethylene dirhodium(I) $(4.9 \mathrm{mg}, \quad 13 \mu \mathrm{mol})$ and tris(4-trifluoromethylphenyl)phosphine $(23.3 \mathrm{mg}, 50.0 \mu \mathrm{mol})$ in dioxane $(0.5 \mathrm{~mL})$ were heated in a reaction tube at $90{ }^{\circ} \mathrm{C}$ for $5 \mathrm{~min}$. The mixture was cooled to room temperature. 2-(2-Thienyl)pyridine (1) (40 mg, $0.25 \mathrm{mmol}$ ), phenylboronic acid (122 mg, $1.00 \mathrm{mmol}$ ), TEMPO, $t$-BuOH $(0.1 \mathrm{~mL})$ and dioxane $(0.5 \mathrm{~mL})$ were added. The reaction tube was carefully evacuated and equipped with a balloon filled with the gas mentioned in Table 1. The reaction mixture was stirred at $90{ }^{\circ} \mathrm{C}$ for $13 \mathrm{~h}$ and filtered through a plug of silica gel (ca. $40 \mathrm{~mL} \mathrm{MTBE}$ as eluent). The filtrate was concentrated under vacuum, and the residue was purified by FC (pentane/EtOAc 100:1) and yielded a mixture of unreacted 2-(2-thienyl)pyridine (1) and phenylated product 2a. The yield was calculated via integration of the obtained ${ }^{1} \mathrm{H}-\mathrm{NMR}$ spectra.

Table 1. Rh-catalyzed C-H phenylation using Oxygen or Air as (Co-)Oxidant (GP3).

\begin{tabular}{lllll}
\hline entry & TEMPO & gas & $\mathbf{1}(\%)$ & $\mathbf{2 a}(\%)$ \\
\hline 1 & $156 \mathrm{mg}, 1.00 \mathrm{mmol}$ & --- & 0 & 88 \\
2 & $7.8 \mathrm{mg}, 50 \mu \mathrm{mol}$ & $\mathrm{O}_{2}$ & 28 & 46 \\
3 & $7.8 \mathrm{mg}, 50 \mu \mathrm{mol}$ & air & 29 & 51 \\
4 & --- & $\mathrm{O}_{2}$ & 46 & 29
\end{tabular}




\section{2-(3-Phenyl-thiophen-2-yl)-pyridine (2a)}

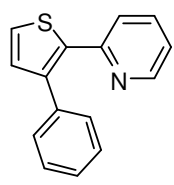

According to GP1 with 2-(2-thienyl)pyridine (1) $(40 \mathrm{mg}, 0.25 \mathrm{mmol})$ and phenylboronic acid (122 mg, $1.00 \mathrm{mmol}$ ). FC (pentane/EtOAc 200:1) gave 2a as yellow oil (59 $\mathrm{mg}, 0.25 \mathrm{mmol}, 99 \%)$.

IR (neat): 3055m, 2970m, 2930m, 1581s, 1568m, 1464s, 1417m, 1289m, 1153m, 1075m, 994m, 883m, 785s, 765s, 700s. ${ }^{1} \mathrm{H}-\mathrm{NMR}\left(300 \mathrm{MHz}, \mathrm{CDCl}_{3}\right): \delta=8.60(d, J=4.5 \mathrm{~Hz}, 1 \mathrm{H}$, $\mathrm{CH}) ; 7.42(d, J=5.1 \mathrm{~Hz}, 1 \mathrm{H}, \mathrm{CH}) ; 7.42-7.32(m, 6 \mathrm{H}, \mathrm{CH}) ; 7.13-7.06(m, 2 \mathrm{H}, \mathrm{CH})$; $7.03(d, J=8.1 \mathrm{~Hz}, 1 \mathrm{H}, \mathrm{CH}) .{ }^{13} \mathrm{C}-\mathrm{NMR}\left(75 \mathrm{MHz}, \mathrm{CDCl}_{3}\right): \delta=152.6(\mathrm{C}), 149.0(\mathrm{CH})$, $140.1(\mathrm{C}), 138.6(\mathrm{C}), 136.7(\mathrm{C}), 136.1(\mathrm{CH}), 131.1(\mathrm{CH}), 129.0(\mathrm{CH}), 128.6(\mathrm{CH}), 127.4$ $(\mathrm{CH}), 126.7(\mathrm{CH}), 122.0(\mathrm{CH}), 121.6(\mathrm{CH})$. MS (EI) m/z: 237 (47, [M] $\left.]^{+}\right), 236(100), 235$ (17), 204 (13), 118 (11). HRMS (ESI): calcd for [M+Na] $]^{+}: 260.0504$; found: 260.0501.

\section{2-(3-p-Tolyl-thiophen-2-yl)-pyridine (2b)}

According to GP1 with 2-(2-thienyl)pyridine (1) $(40 \mathrm{mg}, 0.25 \mathrm{mmol})$ and

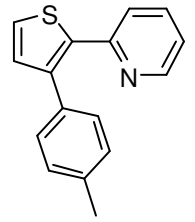

4-methylphenylboronic acid (136 mg, $1.00 \mathrm{mmol}$ ). FC (pentane/EtOAc 200:1) gave $2 \mathbf{b}$ as pale orange oil ( $57 \mathrm{mg}, 0.23 \mathrm{mmol}, 91 \%$ ).

IR (neat): 3051m, 3022m, 2995m, 2972m, 2921m, 2866m, 1581s, 1506m, $1465 s, 1434 m, 1152 m, 995 m, 848 m, 819 m, 781 s .{ }^{1} \mathrm{H}-\mathrm{NMR}\left(300 \mathrm{MHz}, \mathrm{CDCl}_{3}\right): \delta=8.53$ $(d, J=3.4 \mathrm{~Hz}, 1 \mathrm{H}, \mathrm{CH}) ; 7.42-7.26(m, 2 \mathrm{H}, \mathrm{CH}) ; 7.19(d, J=7.6 \mathrm{~Hz}, 2 \mathrm{H}, \mathrm{CH}) ; 7.11(d$, $J=7.5 \mathrm{~Hz}, 2 \mathrm{H}, \mathrm{CH}) ; 7.07-6.96(m, 3 \mathrm{H}, \mathrm{CH}) ; 2.33\left(s, 3 \mathrm{H}, \mathrm{CH}_{3}\right) .{ }^{13} \mathrm{C}-\mathrm{NMR}(75 \mathrm{MHz}$, $\left.\mathrm{CDCl}_{3}\right): \delta=151.5(\mathrm{C}), 147.8(\mathrm{CH}), 138.8(\mathrm{C}), 137.1(\mathrm{C}), 136.0(\mathrm{C}), 134.9(\mathrm{CH}), 132.5$ (C), $130.0(\mathrm{CH}), 128.1(\mathrm{CH}), 127.6(\mathrm{CH}), 125.4(\mathrm{CH}), 120.8(\mathrm{CH}), 120.4(\mathrm{CH}), 19.9$ $\left(\mathrm{CH}_{3}\right)$. MS (EI) m/z: 251 (31, [M] $\left.]^{+}\right), 250$ (100), 235 (9), 125 (6), 118 (4). HRMS (ESI): calcd for $[\mathrm{M}+\mathrm{H}]^{+}: 252.0841$; found: 252.1105 . 


\section{2-[3-(4-Vinyl-phenyl)-thiophen-2-yl]-pyridine (2c)}

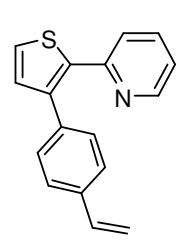

According to GP1 with 2-(2-thienyl)pyridine (1) (40 mg, $0.25 \mathrm{mmol})$ and 4-vinylbenzeneboronic acid $(148 \mathrm{mg}, 1.00 \mathrm{mmol})$. FC (pentane/EtOAc 200:1) gave $2 \mathbf{c}$ as pale orange oil (57 $\mathrm{mg}, 0.22 \mathrm{mmol}, 87 \%)$.

mp: $70{ }^{\circ} \mathrm{C}$. IR (neat): $3064 m, 3003 m, 2933 m, 1628 m, 1582 s, 1504 m, 1465 s$, $1435 m, 1290 m, 994 m, 848 m, 783 s .{ }^{1} \mathrm{H}-\mathrm{NMR}\left(300 \mathrm{MHz}, \mathrm{CDCl}_{3}\right): \delta=8.54(d, J=3.3 \mathrm{~Hz}$, $1 \mathrm{H}, \mathrm{CH}) ; 7.45-7.23(m, 6 \mathrm{H}, \mathrm{CH}) ; 7.10-6.65(m, 3 \mathrm{H}, \mathrm{CH}) ; 6.69$ (dd, J=17.5 Hz, $J=10.8 \mathrm{~Hz}, 1 \mathrm{H}, \mathrm{CH}) ; 5.74\left(d, J=17.5 \mathrm{~Hz}, 1 \mathrm{H}, \mathrm{CH}_{\mathrm{a}} \mathrm{H}\right) ; 5.23(d, J=10.8 \mathrm{~Hz}, 1 \mathrm{H}$, $\left.\mathrm{CH}_{\mathrm{b}} \mathrm{H}\right) .{ }^{13} \mathrm{C}-\mathrm{NMR}\left(75 \mathrm{MHz}, \mathrm{CDCl}_{3}\right): \delta=150.9(\mathrm{C}), 147.8(\mathrm{CH}), 137.8(\mathrm{C}), 137.0(\mathrm{C})$, $134.9(\mathrm{C}), 134.5(\mathrm{CH}), 134.4(\mathrm{C}), 134.4(\mathrm{CH}), 129.2(\mathrm{CH}), 127.4(\mathrm{CH}), 125.0(\mathrm{CH})$, $124.7(\mathrm{CH}), 120.3(\mathrm{CH}), 112.0(\mathrm{CH}), 112.3\left(\mathrm{CH}_{2}\right)$. MS (EI) m/z: $263\left(39,[\mathrm{M}]^{+}\right), 262$ (100), 236 (5), 228 (5), 130 (7). HRMS (ESI): calcd for [M+Na] $]^{+}$: 286.0661; found: 286.0656.

\section{2-(3-Benzo[1,3]dioxol-5-yl-thiophen-2-yl)-pyridine (2d)}

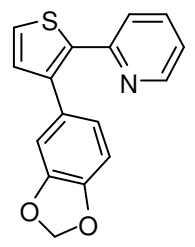

According to GP1 with 2-(2-thienyl)pyridine (1) $(40 \mathrm{mg}, 0.25 \mathrm{mmol})$ and 3,4-methylenedioxybenzeneboronic acid (166 mg, $1.00 \mathrm{mmol})$. FC (pentane/EtOAc 100:1); gave 2d as pale yellow oil (64 mg, $0.23 \mathrm{mmol}$, $91 \%)$.

IR (neat): 3054m, 2890m, 2777m, 1581m, 1501m, 1481s, 1464s, 1435m, 1231s, 1216s, $1034 s, 926 s, 856 m, 779 s, 717 m .{ }^{1} \mathrm{H}-\mathrm{NMR}\left(300 \mathrm{MHz}, \mathrm{CDCl}_{3}\right): \delta=8.59(d, J=4.6 \mathrm{~Hz}$, $1 \mathrm{H}, \mathrm{CH}) ; 7.52-7.43(m, 1 \mathrm{H}, \mathrm{CH}) ; 7.39(d, J=5.1 \mathrm{~Hz}, 1 \mathrm{H}, \mathrm{CH}) ; 7.17-7.07(m, 2 \mathrm{H}$, $\mathrm{CH}) ; 7.03(d, J=5.1 \mathrm{~Hz}, 1 \mathrm{H}, \mathrm{CH}) ; 6.81(s, 3 \mathrm{H}, 3 \times \mathrm{CH}) ; 5.98\left(s, 2 \mathrm{H}, \mathrm{CH}_{2}\right) .{ }^{13} \mathrm{C}-\mathrm{NMR}$ (75 MHz, $\left.\mathrm{CDCl}_{3}\right): \delta=152.5(\mathrm{C}), 148.9(\mathrm{CH}), 147.8(\mathrm{C}), 147.1(\mathrm{C}), 139.8(\mathrm{C}), 138.0(\mathrm{C})$, $136.4(\mathrm{CH}), 131.1(\mathrm{CH}), 130.4(\mathrm{C}), 126.7(\mathrm{CH}), 122.5(\mathrm{CH}), 122.2(\mathrm{CH}), 121.7(\mathrm{CH})$, $109.4(\mathrm{CH}), 108.5(\mathrm{CH}), 101.1\left(\mathrm{CH}_{2}\right)$. MS (EI) m/z: $281\left(46,[\mathrm{M}]^{+}\right), 280(100), 252(6)$, 222 (19), 112 (13). HRMS (ESI): calcd for [M+Na] ${ }^{+}: 304.0403$; found: 304.0397. 


\section{2-[3-(3,5-Bis-trifluoromethyl-phenyl)-thiophen-2-yl]-pyridine (2e)}

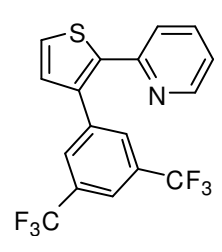

According to GP1 with 2-(2-thienyl)pyridine (1) $(40 \mathrm{mg}, 0.25 \mathrm{mmol})$ and 3,5-bis(trifluoromethyl)benzeneboronic acid $(258 \mathrm{mg}, 1.00 \mathrm{mmol})$. FC (pentane/EtOAc 100:1) gave 2e as colorless solid (86 mg, $0.23 \mathrm{mmol}$, $92 \%)$.

mp: $74{ }^{\circ} \mathrm{C}$. IR (neat): $3065 m, 1618 m, 1583 m, 1466 m, 1348 m, 1434 m, 1275 s, 1172 s$, $1124 s, 1086 m, 997 m .{ }^{1} \mathrm{H}-\mathrm{NMR}\left(300 \mathrm{MHz}, \mathrm{CDCl}_{3}\right): \delta=8.53(b s, 1 \mathrm{H}, \mathrm{CH}) ; 7.75(b s, 3 \mathrm{H}$, $\mathrm{CH}) ; 7.53$ - $7.36(m, 2 \mathrm{H}, \mathrm{CH}) ; 7.21-7.05(m, 2 \mathrm{H}, \mathrm{CH}) ; 6.99(d, J=7.3 \mathrm{~Hz}, 1 \mathrm{H}, \mathrm{CH})$. ${ }^{13} \mathrm{C}-\mathrm{NMR}\left(75 \mathrm{MHz}, \mathrm{CDCl}_{3}\right): \delta=151.3(\mathrm{C}), 149.3(\mathrm{CH}), 139.9(\mathrm{C}), 138.2(\mathrm{C}), 136.0$ $(\mathrm{CH}), 136.0(\mathrm{C}), 131.3\left(q,{ }^{2} J(\mathrm{C}, \mathrm{F})=33 \mathrm{~Hz}, 2 \times \mathrm{C}\right), 129.7(\mathrm{CH}), 128.8\left(q,{ }^{3} J(\mathrm{C}, \mathrm{F})=3 \mathrm{~Hz}\right.$, $2 \times \mathrm{CH}), 127.0(\mathrm{CH}), 122.6\left(q,{ }^{1} J(\mathrm{C}, \mathrm{F})=271 \mathrm{~Hz}, 2 \times \mathrm{C}\right), 122.1(\mathrm{CH}), 122.0(\mathrm{CH}), 120.4$ $\left(\right.$ sept $\left.,{ }^{3} J(\mathrm{C}, \mathrm{F})=4 \mathrm{~Hz}, \mathrm{CH}\right)$. MS (EI) $m / z: 373\left(26,[\mathrm{M}]^{+}\right), 372$ (100), 352 (56), 340 (8), 234 (4). HRMS (ESI): calcd for [M+H] $]^{+}: 374.0433$; found: 374.0439.

\section{2-[3-(3-Chloro-phenyl)-thiophen-2-yl]-pyridine (2f)}

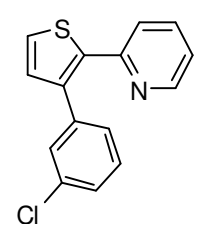

According to GP1 with 2-(2-thienyl)pyridine (1) $(40 \mathrm{mg}, 0.25 \mathrm{mmol})$ and 3-chlorobenzeneboronic acid $(156 \mathrm{mg}, 1.00 \mathrm{mmol})$. FC (pentane/EtOAc 100:1) gave $2 \mathbf{f}$ as pale orange oil (61 $\mathrm{mg}, 0.22 \mathrm{mmol}, 90 \%$ ).

IR (neat): 3061m, 1596m, 1581m, 1564m, 1465s, 1435m, 1152m, 1121m, $1070 m, 996 m, 882 m, 780 s, 726 m .{ }^{1} \mathrm{H}-\mathrm{NMR}\left(300 \mathrm{MHz}, \mathrm{CDCl}_{3}\right): \delta=8.59(d, J=3.9 \mathrm{~Hz}$, $1 \mathrm{H}, \mathrm{CH}) ; 7.52$ - 7.38 (m, $2 \mathrm{H}, \mathrm{CH}) ; 7.38-7.16(m, 4 \mathrm{H}, \mathrm{CH}) ; 7.16-7.08(m, 1 \mathrm{H}, \mathrm{CH})$; 7.08 - 6.98 (m, $2 \mathrm{H}, \mathrm{CH}) .{ }^{13} \mathrm{C}-\mathrm{NMR}\left(75 \mathrm{MHz}, \mathrm{CDCl}_{3}\right): \delta=152.2(\mathrm{C}), 149.2(\mathrm{CH}), 139.1$ (C), $138.5(2 \times \mathrm{C}), 136.4(\mathrm{CH}), 134.5(\mathrm{C}), 130.8(\mathrm{CH}), 129.8(\mathrm{CH}), 129.0(\mathrm{CH}), 127.6$ $(\mathrm{CH}), 127.3(\mathrm{CH}), 127.1(\mathrm{CH}), 122.3(\mathrm{CH}), 122.0(\mathrm{CH}) . \mathrm{MS}(\mathrm{EI}) \mathrm{m} / \mathrm{z}: 271\left(26,[\mathrm{M}]^{+}\right)$, 270 (100), 235 (17), 118 (11), 96 (11). HRMS (ESI): calcd for [M+Na]+: 294.0115; found: 294.0118 . 


\section{2-(3-o-Tolyl-thiophen-2-yl)-pyridine (2g)}

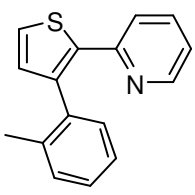

According to GP1 with 2-(2-thienyl)pyridine (1) (40 mg, $0.25 \mathrm{mmol})$ and 2-methylphenylboronic acid $(136 \mathrm{mg}, 1.00 \mathrm{mmol})$. FC (pentane/EtOAc 200:1) gave $2 \mathrm{~g}$ as pale yellow oil (48 $\mathrm{mg}, 0.19 \mathrm{mmol}, 76 \%)$.

IR (neat): 3056m, 3017m, 2921m, 2857m, 1581s, 1563m, 1463s, 1433s, 1416m, 1289s, $1153 m, 1085 m, 994 m, 887 m, 791 s, 760 s, 729 s .{ }^{1} \mathrm{H}-\mathrm{NMR}\left(300 \mathrm{MHz}, \mathrm{CDCl}_{3}\right): \delta=8.57(d$, $J=4.4 \mathrm{~Hz}, 1 \mathrm{H}, \mathrm{CH}) ; 7.44(d, J=5.1 \mathrm{~Hz}, 1 \mathrm{H}, \mathrm{CH}) ; 7.39$ - $7.21(m, 5 \mathrm{H}, \mathrm{CH}) ; 7.10$ - 7.01 $(m, 1 \mathrm{H}, \mathrm{CH}) ; 6.97(d, J=5.1 \mathrm{~Hz}, 1 \mathrm{H}, \mathrm{CH}) ; 6.77(d, J=8.1 \mathrm{~Hz}, \mathrm{CH}) ; 2.11\left(\mathrm{~s}, 3 \mathrm{H}, \mathrm{CH}_{3}\right)$. ${ }^{13} \mathrm{C}-\mathrm{NMR}\left(75 \mathrm{MHz}, \mathrm{CDCl}_{3}\right): \delta=152.3(\mathrm{C}), 148.7(\mathrm{CH}), 139.2(\mathrm{C}), 139.0(\mathrm{C}), 136.6(\mathrm{C})$, $136.0(\mathrm{C}), 135.9(\mathrm{CH}), 130.8(\mathrm{CH}), 130.0(\mathrm{CH}), 129.2(\mathrm{CH}), 127.5(\mathrm{CH}), 126.1(\mathrm{CH})$, $125.8(\mathrm{CH}), 121.1(\mathrm{CH}), 119.5(\mathrm{CH}), 19.4(\mathrm{CH}) . \mathrm{MS}(\mathrm{EI}) \mathrm{m} / z: 251\left(9,[\mathrm{M}]^{+}\right), 236(100)$, 218 (16), 204 (7), 171 (4), 125 (8), 78 (8). HRMS (ESI): calcd for $[\mathrm{M}+\mathrm{H}]^{+}: 252.0841$; found: 252.0839.

\section{2-[3-(2-Ethoxy-phenyl)-thiophen-2-yl]-pyridine (2h)}

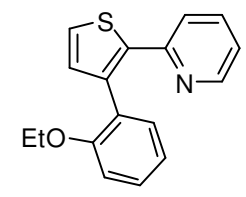

According to GP1 with 2-(2-thienyl)pyridine (1) (40 mg, $0.25 \mathrm{mmol})$ and 2-ethoxyphenylboronic acid (166 mg, $1.00 \mathrm{mmol})$. FC (pentane/EtOAc 60:1) gave $\mathbf{2 h}$ as yellow oil (58 $\mathrm{mg}, 0.21 \mathrm{mmol}, 82 \%)$.

IR (neat): 3056m, 2980m, 2928m, 1581m, 1492m, 1465s, 1446m, 1239s, 1043s, 907m, $882 m, 783 s, 725 m .{ }^{1} \mathrm{H}-\mathrm{NMR}\left(300 \mathrm{MHz}, \mathrm{CDCl}_{3}\right): \delta=8.60(d, J=4.7 \mathrm{~Hz}, 1 \mathrm{H}, \mathrm{CH}) ; 7.47$ - $7.38(m, 2 \mathrm{H}, \mathrm{CH}) ; 7.38$ - $7.26(m, 2 \mathrm{H}, \mathrm{CH}) ; 7.14$ - 7.07 (m, 2 H, CH); 7.03 (d, $J=8.4 \mathrm{~Hz}, 1 \mathrm{H}, \mathrm{CH}) ; 7.00(t, J=7.2 \mathrm{~Hz}, 1 \mathrm{H}, \mathrm{CH}) ; 6.93(d, J=8.4 \mathrm{~Hz}, 1 \mathrm{H}, \mathrm{CH}) ; 3.85$ $\left(q, J=6.9 \mathrm{~Hz}, 2 \mathrm{H}, \mathrm{CH}_{2}\right) ; 1.03\left(t, J=7.0 \mathrm{~Hz}, 3 \mathrm{H}, \mathrm{CH}_{3}\right) .{ }^{13} \mathrm{C}-\mathrm{NMR}\left(75 \mathrm{MHz}, \mathrm{CDCl}_{3}\right)$ : $\delta=155.9(\mathrm{C}), 153.2(\mathrm{C}), 148.4(\mathrm{CH}), 139.2(\mathrm{C}), 136.1(\mathrm{CH}), 136.1(\mathrm{C}), 131.9(\mathrm{CH})$, $131.1(\mathrm{CH}), 129.1(\mathrm{CH}), 126.0(\mathrm{CH}), 126.0(\mathrm{C}), 121.4(\mathrm{CH}), 121.0(\mathrm{CH}), 120.7(\mathrm{CH})$, $112.4(\mathrm{CH}), 63.6\left(\mathrm{CH}_{2}\right), 14.3\left(\mathrm{CH}_{3}\right) . \mathrm{MS}(\mathrm{EI}) \mathrm{m} / z: 281\left(1,[\mathrm{M}]^{+}\right), 252$ (6), $263(100), 223$ (14), 191 (4), 78 (3). HRMS (ESI): calcd for [M+Na] $]^{+}$304.0767; found: 304.0773. 


\section{2-(3-Ethoxy-biphenyl-2-yl)-pyridine (4a)}

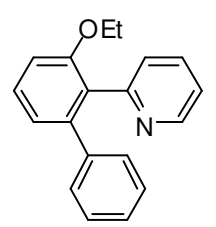

According to GP1 with 2-(2-ethoxyphenyl)pyridine (3) (50 mg, $0.25 \mathrm{mmol}$ ) and phenylboronic acid (122 mg, $1.00 \mathrm{mmol})$. FC (pentane/MTBE 5:1) gave $4 \mathbf{a}$ as pale yellow oil (51 $\mathrm{mg}, 0.19 \mathrm{mmol}, 74 \%)$.

IR (neat): 3058m, 2979m, 2930m, 2880m, 1587s, 1570s, 1457s, 1423s, 1309m, 1252s, 1122s, 1043m, 1024m, 942m, 800m. ${ }^{1} \mathrm{H}-\mathrm{NMR}$ (300 MHz, $\mathrm{CDCl}_{3}$ ): $\delta=8.56(d, J=4.0 \mathrm{~Hz}, 1 \mathrm{H}, \mathrm{CH}) ; 7.52(t, J=7.7 \mathrm{~Hz}, 1 \mathrm{H}, \mathrm{CH}) ; 7.47-7.35(m, 1 \mathrm{H}, \mathrm{CH})$; 7.18 - $7.03(m, 8 \mathrm{H}, \mathrm{CH}) ; 7.00(d, J=8.3 \mathrm{~Hz}, 1 \mathrm{H}, \mathrm{CH}) ; 4.18$ - $3.92\left(m, 2 \mathrm{H}, \mathrm{CH}_{2}\right) ; 1.32$ $1.16\left(m, 3 \mathrm{H}, \mathrm{CH}_{3}\right) .{ }^{13} \mathrm{C}-\mathrm{NMR}\left(75 \mathrm{MHz}, \mathrm{CDCl}_{3}\right): \delta=156.6(\mathrm{C}), 156.3(\mathrm{C}), 147.8(\mathrm{CH})$, $142.8(\mathrm{C}), 140.9(\mathrm{C}), 135.9(\mathrm{CH}), 129.5(\mathrm{CH}), 129.3(\mathrm{CH}), 128.5(\mathrm{C}), 127.6(\mathrm{CH}), 126.5$ $(\mathrm{CH}), 126.3(\mathrm{CH}), 122.4(\mathrm{CH}), 121.3(\mathrm{CH}), 111.5(\mathrm{CH}), 64.4\left(\mathrm{CH}_{2}\right), 14.6\left(\mathrm{CH}_{3}\right)$. MS (EI) m/z: 275 (77, [M] $\left.{ }^{+}\right), 274$ (100), 260 (57), 246 (64), 230 (86), 217 (48), 202 (10), 189 (12). HRMS (ESI): calcd for $[\mathrm{M}+\mathrm{H}]^{+}: 276.1383$; found: 276.1381 .

\section{2-(3-Ethoxy-4'-methyl-biphenyl-2-yl)-pyridine (4b)}

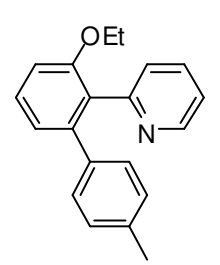

According to GP1 with 2-(2-ethoxyphenyl)pyridine (3) (50 mg, $0.25 \mathrm{mmol}$ ) and 4-methylphenylboronic acid (136 mg, $1.00 \mathrm{mmol}$ ). FC (pentane/ EtOAc 10:1) gave $\mathbf{4 b}$ as pale yellow solid (48 $\mathrm{mg}, 0.17 \mathrm{mmol}, 66 \%$ ).

mp: $79^{\circ} \mathrm{C}$. IR (neat): $3053 m, 2979 m, 2924 m, 1589 s, 1515 m, 1457 s, 1425 m$, $1253 s, 1123 s, 1042 m, 941 m, 797 s .{ }^{1} \mathrm{H}-\mathrm{NMR}\left(300 \mathrm{MHz}, \mathrm{CDCl}_{3}\right): \delta=8.58(b s, 1 \mathrm{H}, \mathrm{CH})$; $7.52(t, J=7.1 \mathrm{~Hz}, 1 \mathrm{H}, \mathrm{CH}) ; 7.39(t, J=7.9 \mathrm{~Hz}, 1 \mathrm{H}, \mathrm{CH}) ; 7.15-6.90(m, 8 \mathrm{H}, \mathrm{CH})$; $4.04\left(q, J=6.8 \mathrm{~Hz}, 2 \mathrm{H}, \mathrm{CH}_{2}\right) ; 2.26\left(s, 3 \mathrm{H}, \mathrm{CH}_{3}\right) ; 1.23\left(t, J=6.8 \mathrm{~Hz}, 3 \mathrm{H}, \mathrm{CH}_{3}\right)$. ${ }^{13} \mathrm{C}-\mathrm{NMR}\left(75 \mathrm{MHz}, \mathrm{CDCl}_{3}\right): \delta=156.6(2 \times \mathrm{C}), 148.0(\mathrm{CH}), 142.6(\mathrm{C}), 138.0(\mathrm{C}), 135.8$ $(\mathrm{C}), 135.7(\mathrm{CH}), 129.3(\mathrm{CH}), 129.2(\mathrm{CH}), 128.7(\mathrm{C}), 128.3(\mathrm{CH}), 126.4(\mathrm{CH}), 122.5$ $(\mathrm{CH}), 121.2(\mathrm{CH}), 111.3(\mathrm{CH}), 64.4\left(\mathrm{CH}_{2}\right), 20.9\left(\mathrm{CH}_{3}\right), 14.6\left(\mathrm{CH}_{3}\right)$. MS (EI) m/z: 289 (80, [M] $]^{+}$), 288 (100), 274 (43), 260 (51), 244 (66), 231 (20), 217 (13). HRMS (ESI): calcd for $[\mathrm{M}+\mathrm{H}]^{+}: 290.1539$; found: 290.1537 . 


\section{2-(3-Ethoxy-4'-vinyl-biphenyl-2-yl)-pyridine (4c)}

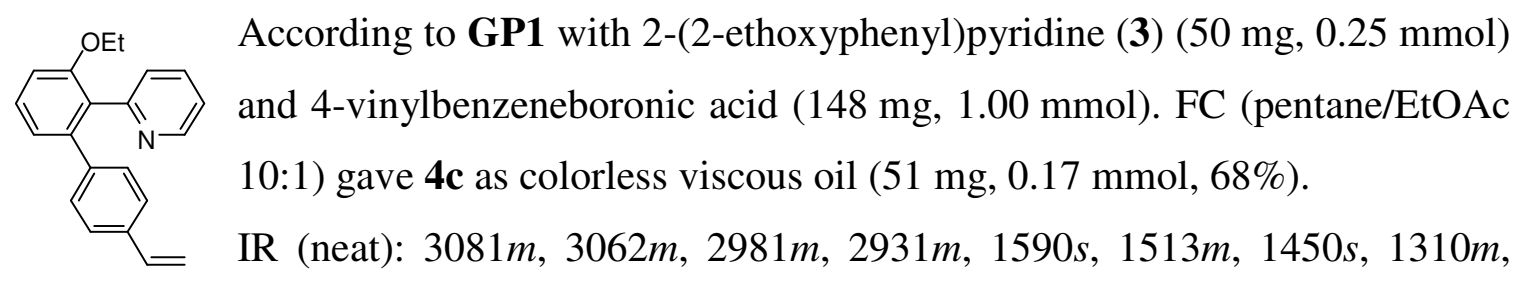

$1251 s, 1123 s, 1040 s, 990 m, 941 m, 910 m, 800 s .{ }^{1} \mathrm{H}-\mathrm{NMR}\left(300 \mathrm{MHz}, \mathrm{CDCl}_{3}\right): \delta=8.62(s$, $1 \mathrm{H}, \mathrm{CH}) ; 7.62(b s, 1 \mathrm{H}, \mathrm{CH}) ; 7.42(t, J=7.9 \mathrm{~Hz}, 1 \mathrm{H}, \mathrm{CH}) ; 7.27-7.15(m, 3 \mathrm{H}, \mathrm{CH})$; $7.12(d, J=7.2 \mathrm{~Hz}, 1 \mathrm{H}, \mathrm{CH}) ; 7.08-6.95(m, 4 \mathrm{H}, \mathrm{CH}) ; 6.62(d d, J=17.6 \mathrm{~Hz}$, $J=10.9 \mathrm{~Hz}, 1 \mathrm{H}, \mathrm{CH}) ; 5.67\left(d, J=17.6 \mathrm{~Hz}, 1 \mathrm{H}, \mathrm{CH}_{\mathrm{a}} \mathrm{H}\right) ; 5.19(d, J=10.8 \mathrm{~Hz}, 1 \mathrm{H}$, $\left.\mathrm{CH}_{\mathrm{b}} \mathrm{H}\right) ; 4.07\left(q, J=6.5 \mathrm{~Hz}, 2 \mathrm{H}, \mathrm{CH}_{2}\right) ; 1.25\left(t, \mathrm{~J}=6.7 \mathrm{~Hz}, 3 \mathrm{H}, \mathrm{CH}_{3}\right) .{ }^{13} \mathrm{C}-\mathrm{NMR}$ $\left(75 \mathrm{MHz}, \mathrm{CDCl}_{3}\right): \delta=156.7(\mathrm{C}), 155.9(\mathrm{C}), 147.4(\mathrm{CH}), 142.4(\mathrm{C}), 140.4(\mathrm{C}), 136.7$ $(\mathrm{CH}), 136.4(\mathrm{CH}), 135.7(\mathrm{C}), 129.7(2 \times \mathrm{CH}), 127.7(\mathrm{C}), 126.9(\mathrm{CH}), 125.6(\mathrm{CH}), 122.4$ $(\mathrm{CH}), 121.6(\mathrm{CH}), 113.6\left(\mathrm{CH}_{2}\right), 111.6(\mathrm{CH}), 64.5\left(\mathrm{CH}_{2}\right), 14.7\left(\mathrm{CH}_{3}\right) . \mathrm{MS}(\mathrm{EI}) \mathrm{m} / z: 301$ (100, [M] $]^{+}$), 300 (100), 286 (45), 272 (50), 256 (64), 243 (26), 241 (16). HRMS (ESI): calcd for $[\mathrm{M}+\mathrm{H}]^{+}:$302.1539; found: 302.1540 .

\section{2-(3-Ethoxy-4'-methoxy-biphenyl-2-yl)-pyridine (4d)}

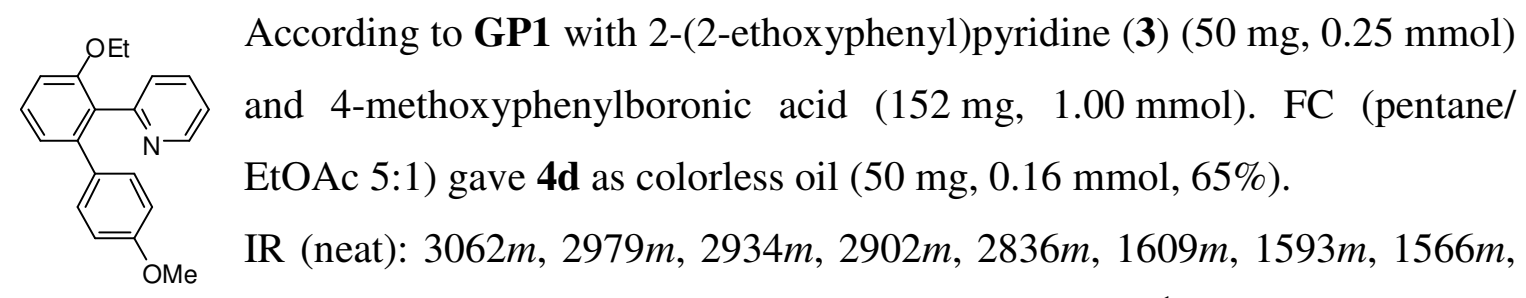
$1515 s, 1457 s, 1290 m, 1244 s, 1176 m, 1124 s, 1026 m, 942 m, 798 s .{ }^{1} \mathrm{H}-\mathrm{NMR}(300 \mathrm{MHz}$, $\left.\mathrm{CDCl}_{3}\right): \delta=8.61(b s, 1 \mathrm{H}, \mathrm{CH}) ; 7.83-7.53(m, 1 \mathrm{H}, \mathrm{CH}) ; 7.39(t, J=7.7 \mathrm{~Hz}, 1 \mathrm{H}, \mathrm{CH})$; 7.28 - $7.13(m, 1 \mathrm{H}, \mathrm{CH}) ; 7.09(d, J=6.6 \mathrm{~Hz}, 1 \mathrm{H}, \mathrm{CH}) ; 7.06-6.93(m, 4 \mathrm{H}, \mathrm{CH}) ; 6.68$ $(d, J=7.8 \mathrm{~Hz}, 2 \mathrm{H}, \mathrm{CH}) ; 4.05\left(q, J=6.6 \mathrm{~Hz}, 2 \mathrm{H}, \mathrm{CH}_{2}\right) ; 3.73\left(s, 3 \mathrm{H}, \mathrm{CH}_{3}\right) ; 1.24(t$, $\left.J=6.2 \mathrm{~Hz}, 3 \mathrm{H}, \mathrm{CH}_{3}\right) .{ }^{13} \mathrm{C}-\mathrm{NMR}\left(75 \mathrm{MHz}, \mathrm{CDCl}_{3}\right): \delta=158.3(\mathrm{C}), 156.7(\mathrm{C}), 156.0(\mathrm{C})$, $147.2(\mathrm{CH}), 142.4(\mathrm{C}), 136.8(\mathrm{CH}), 133.2(\mathrm{C}), 130.6(\mathrm{CH}), 129.7(\mathrm{CH}), 127.5(\mathrm{C}), 127.0$ $(\mathrm{CH}), 122.5(\mathrm{CH}), 121.6(\mathrm{CH}), 113.2(\mathrm{CH}), 111.2(\mathrm{CH}), 64.5\left(\mathrm{CH}_{2}\right), 55.1\left(\mathrm{CH}_{3}\right), 14.7$ $\left(\mathrm{CH}_{3}\right)$. MS (EI) m/z: 306 (15, [M] $\left.{ }^{+}\right), 304$ (100), 290 (94), 260 (35), 233 (21), 204 (29). HRMS (ESI): calcd for $[\mathrm{M}+\mathrm{H}]^{+}: 306.1489$; found: 306.1496. 


\section{2-(3-Ethoxy-4'-fluoro-biphenyl-2-yl)-pyridine (4e)}

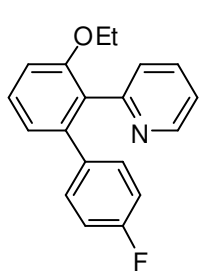

According to GP1 with 2-(2-ethoxyphenyl)pyridine (3) (50 mg, $0.25 \mathrm{mmol}$ ) and 4-fluorophenylboronic acid (140 mg, $1.00 \mathrm{mmol}$ ). FC (pentane/EtOAc 10:1) gave 4e as off white solid (55 $\mathrm{mg}, 0.19 \mathrm{mmol}, 75 \%)$.

mp: $91{ }^{\circ} \mathrm{C}$. IR (neat): 3043m, 2980m, 2931m, 2884m, 2055w, 1979w, $1891 w, 1846 w, 1772 w, 1598 m, 1574 m, 1510 s, 1453 s, 1251 s, 1218 s, 1158 m, 1122 s$, $1039 m, 943 m, 798 s, 742 s .{ }^{1} \mathrm{H}-\mathrm{NMR}\left(300 \mathrm{MHz}, \mathrm{CDCl}_{3}\right): \delta=8.57$ (bs, $\left.1 \mathrm{H}, \mathrm{CH}\right) ; 7.75-$ $7.33(m, 2 \mathrm{H}, \mathrm{CH}) ; 7.21-6.92(m, 6 \mathrm{H}, \mathrm{CH}) ; 6.90-6.73(m, 2 \mathrm{H}, \mathrm{CH}) ; 4.04(b r, 2 \mathrm{H}$, $\left.\mathrm{CH}_{2}\right) ; 1.23\left(b r, 3 \mathrm{H}, \mathrm{CH}_{3}\right) .{ }^{13} \mathrm{C}-\mathrm{NMR}\left(75 \mathrm{MHz}, \mathrm{CDCl}_{3}\right): \delta=161.5\left(d,{ }^{l} J(\mathrm{C}, \mathrm{F})=244 \mathrm{~Hz}\right.$, C), $156.6(\mathrm{C}), 156.0(\mathrm{C}), 147.8(\mathrm{CH}), 141.7(\mathrm{C}), 136.8\left(d,{ }^{4} \mathrm{~J}(\mathrm{C}, \mathrm{F})=3 \mathrm{~Hz}, \mathrm{C}\right), 136.2$ $(\mathrm{CH}), 131.0\left(d,{ }^{3} \mathrm{~J}(\mathrm{C}, \mathrm{F})=8 \mathrm{~Hz}, 2 \times \mathrm{CH}\right), 129.5(\mathrm{CH}), 128.3(\mathrm{C}), 126.6(\mathrm{CH}), 122.3(\mathrm{CH})$, $121.5(\mathrm{CH}), 114.5\left(d,{ }^{2} J(\mathrm{C}, \mathrm{F})=21 \mathrm{~Hz}, 2 \times \mathrm{CH}\right), 111.6(\mathrm{CH}), 64.5\left(\mathrm{CH}_{2}\right), 14.6\left(\mathrm{CH}_{3}\right) . \mathrm{MS}$ (EI) m/z: 293 (75, [M] $\left.]^{+}\right), 278$ (73), 264 (64), 248 (100), 235 (40). HRMS (ESI): calcd for $[\mathrm{M}+\mathrm{H}]^{+}:$294.1289; found: 294.1292.

\section{1-(3'-Ethoxy-2'-pyridin-2-yl-biphenyl-3-yl)-ethanone (4f)}

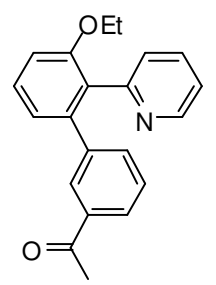

According to GP1 with 2-(2-ethoxyphenyl)pyridine (3) (50 mg, $0.25 \mathrm{mmol}$ )

and 3-acetylphenylboronic acid (164 mg, $1.00 \mathrm{mmol}$ ). FC (pentane/EtOAc $5: 1 \rightarrow 3: 1)$ gave $4 \mathbf{f}$ as yellow oil (45 $\mathrm{mg}, 0.14 \mathrm{mmol}, 57 \%)$.

IR (neat): 3065m, 2981m, 2931m, 1684s, 1598m, 1584m, 1451s, 1322s, $1288 m, 1129 m, 1062 s, 793 m .{ }^{1} \mathrm{H}-\mathrm{NMR}\left(300 \mathrm{MHz}, \mathrm{CDCl}_{3}\right): \delta=8.55(d, J=3.6 \mathrm{~Hz}, 1 \mathrm{H}$, $\mathrm{CH}) ; 7.70(d, J=7.5 \mathrm{~Hz}, 1 \mathrm{H}, \mathrm{CH}) ; 7.67-7.59(m, 2 \mathrm{H}, \mathrm{CH}) ; 7.41(t, J=8.0 \mathrm{~Hz}, 1 \mathrm{H}$, $\mathrm{CH}) ; 7.31-7.17(m, 3 \mathrm{H}, \mathrm{CH}) ; 7.14(d, J=7.8 \mathrm{~Hz}, 1 \mathrm{H}, \mathrm{CH}) ; 7.01(t, J=7.7 \mathrm{~Hz}, 2 \mathrm{H}$, $\mathrm{CH}) ; 4.03\left(q, J=6.9 \mathrm{~Hz}, 2 \mathrm{H}, \mathrm{CH}_{2}\right) ; 2.39\left(s, 3 \mathrm{H}, \mathrm{CH}_{3}\right) ; 1.21\left(t, J=6.9 \mathrm{~Hz}, 3 \mathrm{H}, \mathrm{CH}_{3}\right)$. ${ }^{13} \mathrm{C}-\mathrm{NMR}\left(75 \mathrm{MHz}, \mathrm{CDCl}_{3}\right.$ ): $\delta=197.8(\mathrm{C}), 156.8(\mathrm{C}), 154.8(\mathrm{C}), 146.4(\mathrm{CH}), 141.8(\mathrm{C})$, $140.8(\mathrm{C}), 137.8(\mathrm{CH}), 137.8(\mathrm{C}), 136.6(\mathrm{C}), 134.0(\mathrm{CH}), 130.4(\mathrm{CH}), 129.8(\mathrm{CH}), 128.2$ $(\mathrm{CH}), 127.4(\mathrm{CH}), 126.3(\mathrm{CH}), 122.3(\mathrm{CH}), 122.1(\mathrm{CH}), 111.9(\mathrm{CH}), 64.5\left(\mathrm{CH}_{2}\right), 26.5$ $\left(\mathrm{CH}_{3}\right), 14.5\left(\mathrm{CH}_{3}\right) . \mathrm{MS}(\mathrm{EI}) \mathrm{m} / z: 317$ (70, [M] $\left.]^{+}\right), 316$ (100), 302 (58), 288 (30), 272 (48), 244 (12), 230 (14), 217 (18). HRMS (ESI): calcd for $[\mathrm{M}+\mathrm{H}]^{+}$: 318.1488; found: 318.1489 . 


\section{2-(3'-Chloro-3-ethoxy-biphenyl-2-yl)-pyridine (4g)}

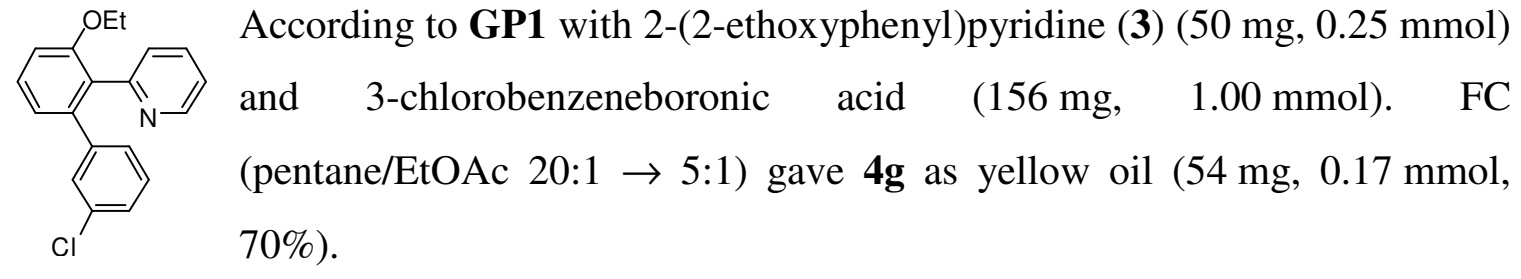

IR (neat): 3063m, 2980m, 2931m, 1578s, 1561s, 1451s, 1391m, 1246s, 1126s, 1040s, 779 s. ${ }^{1} \mathrm{H}-\mathrm{NMR}\left(300 \mathrm{MHz}, \mathrm{CDCl}_{3}\right): \delta=8.70$ (bs, $\left.1 \mathrm{H}, \mathrm{CH}\right) ; 7.82(b s, 1 \mathrm{H}, \mathrm{CH}) ; 7.58-$ $6.98(m, 2 \mathrm{H}, \mathrm{CH}) ; 4.26-3.98\left(m, 2 \mathrm{H}, \mathrm{CH}_{2}\right) ; 1.39-1.15\left(m, 3 \mathrm{H}, \mathrm{CH}_{3}\right) .{ }^{13} \mathrm{C}-\mathrm{NMR}$ $\left(75 \mathrm{MHz}, \mathrm{CDCl}_{3}\right): \delta=156.7(\mathrm{C}), 155.1(\mathrm{C}), 147.3(\mathrm{CH}), 142.5$ (C), 141.4 (C), 137.1 $(\mathrm{CH}), 133.6(\mathrm{C}), 130.0(\mathrm{CH}), 129.4(\mathrm{CH}), 129.0(\mathrm{CH}), 127.9(\mathrm{CH}), 127.3(\mathrm{C}), 127.0$ $(\mathrm{CH}), 126.7(\mathrm{CH}), 122.3(\mathrm{CH}), 122.0(\mathrm{CH}), 112.1(\mathrm{CH}), 64.6\left(\mathrm{CH}_{2}\right), 14.7\left(\mathrm{CH}_{3}\right) . \mathrm{MS}(\mathrm{EI})$ m/z: 311 (26), 310 (39), 309 (87, [M] $]^{+}$), 308 (87), 294 (95), 280 (55), 264 (100), 251 (19), 230 (13), 217 (27), 189 (15). HRMS (ESI): calcd for $[\mathrm{M}+\mathrm{H}]^{+}$: 310.0993; found: 310.0991 .

\section{2-[2-Ethoxy-6-((E)-styryl)-phenyl]-pyridine (4h)}

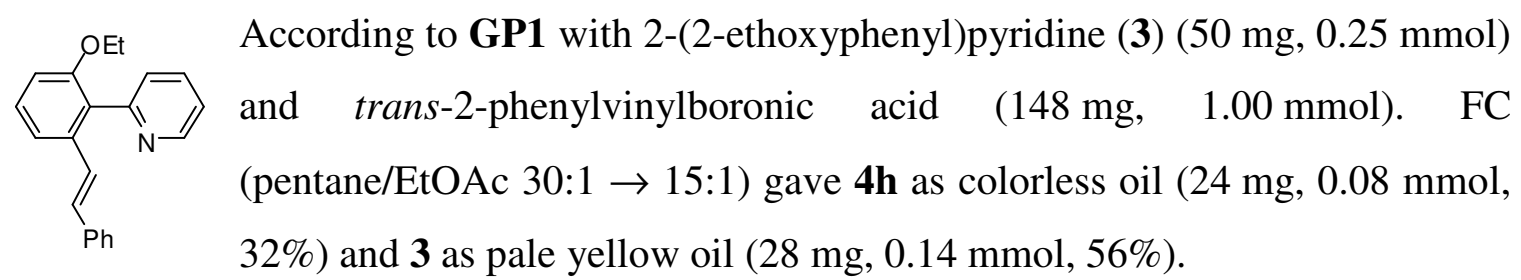

IR (neat): 3057m, 3036m, 2980m, 2931m, 2884m, 1587s, 1577s, 1462s, 1451s, 1265s, $1248 s, 1114 m, 1061 s, 963 m, 794 m .{ }^{1} \mathrm{H}-\mathrm{NMR}\left(300 \mathrm{MHz}, \mathrm{CDCl}_{3}\right): \delta=8.66(d, J=4.6 \mathrm{~Hz}$, $1 \mathrm{H}, \mathrm{CH}) ; 7.71(t, J=7.7 \mathrm{~Hz}, 1 \mathrm{H}, \mathrm{CH}) ; 7.38-7.19(m, 4 \mathrm{H}, \mathrm{CH}) ; 7.19-7.05(m, 5 \mathrm{H}$, $\mathrm{CH}) ; 6.93$ - $6.74(m, 2 \mathrm{H}, \mathrm{CH}) ; 6.73$ - $6.53(m, 1 \mathrm{H}, \mathrm{CH}) ; 3.99$ - $3.83\left(m, 2 \mathrm{H}, \mathrm{CH}_{2}\right) ; 1.15$ $1.06\left(m, 3 \mathrm{H}, \mathrm{CH}_{3}\right) .{ }^{13} \mathrm{C}-\mathrm{NMR}\left(75 \mathrm{MHz}, \mathrm{CDCl}_{3}\right): \delta=156.7(\mathrm{C}), 155.4(\mathrm{C}), 147.9(\mathrm{CH})$, $137.7(\mathrm{C}), 137.4(\mathrm{C}), 136.9(\mathrm{CH}), 136.9(\mathrm{C}), 130.6(\mathrm{CH}), 129.7(\mathrm{CH}), 128.5(\mathrm{CH}), 127.6$ $(\mathrm{CH}), 127.0(\mathrm{CH}), 126.7(\mathrm{CH}), 126.6(\mathrm{CH}), 122.1(\mathrm{CH}), 118.2(\mathrm{CH}), 111.7(\mathrm{CH}), 64.5$ $\left(\mathrm{CH}_{2}\right), 14.6\left(\mathrm{CH}_{3}\right) . \mathrm{MS}(\mathrm{EI}) \mathrm{m} / z: 301\left(69,[\mathrm{M}]^{+}\right), 272$ (12), 244 (13), 224 (100), 196 (35), 167 (19). HRMS (ESI): calcd for [M+H] $]^{+}: 302.1539$; found: 302.1546. 


\section{2-Biphenyl-2-yl-pyridine (6) and 2-[1,1';3',1']terphenyl-2'-yl-pyridine (7)}

According to GP1 with 2-phenylpyridine (5) $(36 \mu \mathrm{L}, 0.25 \mathrm{mmol})$ and phenylboronic acid $(122 \mathrm{mg}, 1.00 \mathrm{mmol}$ ). FC (pentane/MTBE 60:1 $\rightarrow$ 30:1) gave 6 as pale yellow solid (29 mg, $0.13 \mathrm{mmol}, 50 \%)$ and 7 as pale yellow solid (14 mg, $0.05 \mathrm{mmol}, 18 \%)$.

${ }^{1} \mathrm{H}-\mathrm{NMR}\left(300 \mathrm{MHz}, \mathrm{CDCl}_{3}\right): \delta=8.61(d, J=4.5 \mathrm{~Hz}, 1 \mathrm{H}, \mathrm{CH}) ; 7.75-7.62$

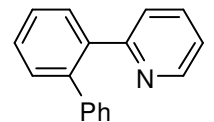

$(m, 1 \mathrm{H}, \mathrm{CH}) ; 7.48$ - $7.32(m, 4 \mathrm{H}, \mathrm{CH}) ; 7.24-7.02(m, 6 \mathrm{H}, \mathrm{CH}) ; 6.85(d$, $J=7.9 \mathrm{~Hz}, 1 \mathrm{H}, \mathrm{CH})$. The spectroscopic data obtained are in accordance with those described in the literature. ${ }^{3}$

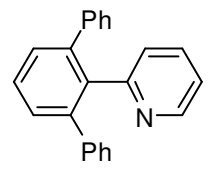

${ }^{1} \mathrm{H}-\mathrm{NMR}\left(300 \mathrm{MHz}, \mathrm{CDCl}_{3}\right): \delta=8.32(d, J=3.5 \mathrm{~Hz}, 1 \mathrm{H}, \mathrm{CH}) ; 7.54-7.37$ $(m, 4 \mathrm{H}, \mathrm{CH}) ; 7.15-7.01(m, 11 \mathrm{H}, \mathrm{CH}) ; 6.94(d, J=7.80 \mathrm{~Hz}, 1 \mathrm{H}, \mathrm{CH})$. The spectroscopic data obtained are in accordance with those described in the literature. ${ }^{3}$

\section{3-Methoxy-biphenyl-2-carbaldehyde (9a)}

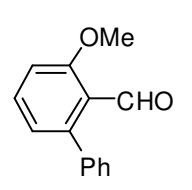

According to GP2 with $N$-(2-methoxybenzylidene)aniline (8) $(53 \mathrm{mg}$, $0.25 \mathrm{mmol})$ and phenylboronic acid $(183 \mathrm{mg}, 1.50 \mathrm{mmol}) . \quad \mathrm{FC}$ (pentane/MTBE 15:1) gave 9a as yellow oil (27 mg, $0.13 \mathrm{mmol}, 51 \%$ ).

IR (neat): 3059w, 3016m, 2967w, 2940m, 2839m, 2760m, 1690s, 1586s, 1570s, 1466s, 1405m, 1279m, 1253s, 1183m, 1118m, 1016s, 882m, 796s. ${ }^{1} \mathrm{H}-\mathrm{NMR}\left(300 \mathrm{MHz}, \mathrm{CDCl}_{3}\right.$ ): $\delta=10.10(s, 1 \mathrm{H}, \mathrm{CHO}) ; 7.52(t, J=8.0 \mathrm{~Hz}, 1 \mathrm{H}, \mathrm{CH}) ; 7.45-7.36(m, 3 \mathrm{H}, \mathrm{CH}) ; 7.34$ $7.28(m, 2 \mathrm{H}, \mathrm{CH}) ; 7.01(d, J=8.4 \mathrm{~Hz}, 1 \mathrm{H}, \mathrm{CH}) ; 6.97(d, J=7.6 \mathrm{~Hz}, 1 \mathrm{H}, \mathrm{CH}) ; 3.95(s$, $\left.3 \mathrm{H}, \mathrm{CH}_{3}\right) .{ }^{13} \mathrm{C}-\mathrm{NMR}\left(75 \mathrm{MHz}, \mathrm{CDCl}_{3}\right): \delta=191.4(\mathrm{CHO}), 160.2(\mathrm{C}), 146.6(\mathrm{C}), 139.0$ $(\mathrm{C}), 133.7(\mathrm{CH}), 129.5(\mathrm{CH}), 128.1(\mathrm{CH}), 127.7(\mathrm{CH}), 123.6(\mathrm{C}), 123.1(\mathrm{CH}), 110.7$ (CH), $56.0\left(\mathrm{CH}_{3}\right)$. MS (EI) m/z: $212\left(100,[\mathrm{M}]^{+}\right), 196$ (28), 180 (31), 168 (28), 152 (42), 139 (31), 115 (19). HRMS (ESI): calcd for [M+Na] ${ }^{+}: 235.0730$; found: 235.0734. 


\section{4'-Fluoro-3-methoxy-biphenyl-2-carbaldehyde (9b)}

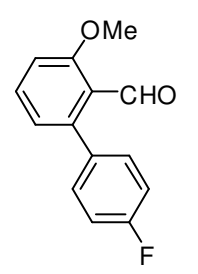

According to GP2 with $N$-(2-methoxybenzylidene)aniline (8) (53 mg, $0.25 \mathrm{mmol}$ ) and 4-fluorophenylboronic acid (210 $\mathrm{mg}, 1.50 \mathrm{mmol})$. FC (pentane/MTBE 10:1) gave 9b as yellow oil (28 mg, $0.12 \mathrm{mmol}, 49 \%$ ). mp: $102{ }^{\circ} \mathrm{C}$. IR (neat): 3069w, 3014w, 2942m, 2843m, 2768m, 1693s, 1596m, $1575 m, 1511 s, 1468 s, 1404 m, 1302 m, 1277 m, 1255 s, 1222 m, 1159 m, 1118 s, 1026 m$, $839 m, 794 m .{ }^{1} \mathrm{H}-\mathrm{NMR}\left(300 \mathrm{MHz}, \mathrm{CDCl}_{3}\right): \delta=10.16(s, 1 \mathrm{H}, \mathrm{CHO}) ; 7.52(t, J=8.0 \mathrm{~Hz}$, $1 \mathrm{H}, \mathrm{CH}) ; 7.32-7.23(m, 2 \mathrm{H}, \mathrm{CH}) ; 7.15-7.05(m, 2 \mathrm{H}, \mathrm{CH}) ; 7.01(d, J=8.4 \mathrm{~Hz}, 1 \mathrm{H}$, $\mathrm{CH}) ; 6.91(d, J=7.6 \mathrm{~Hz}, 1 \mathrm{H}, \mathrm{CH}) ; 3.95\left(s, 3 \mathrm{H}, \mathrm{CH}_{3}\right) .{ }^{13} \mathrm{C}-\mathrm{NMR}\left(75 \mathrm{MHz}, \mathrm{CDCl}_{3}\right)$ : $\delta=191.2(\mathrm{CHO}), 162.5\left(d,{ }^{1} J(\mathrm{C}, \mathrm{F})=246 \mathrm{~Hz}, \mathrm{C}\right), 160.7(\mathrm{C}), 145.1(\mathrm{C}), 135.2\left(d,{ }^{4} J(\mathrm{C}, \mathrm{F})\right.$ $=3 \mathrm{~Hz}, \mathrm{C}), 133.9(\mathrm{CH}), 131.0\left(d,{ }^{3} J(\mathrm{C}, \mathrm{F})=8 \mathrm{~Hz}, 2 \times \mathrm{CH}\right), 123.6(\mathrm{C}), 123.3(\mathrm{CH}), 115.1$ $\left(d,{ }^{2} J(\mathrm{C}, \mathrm{F})=21 \mathrm{~Hz}, 2 \times \mathrm{CH}\right), 110.9(\mathrm{CH}), 56.07\left(\mathrm{CH}_{3}\right) . \mathrm{MS}(\mathrm{EI}) \mathrm{m} / z: 230\left(100,[\mathrm{M}]^{+}\right)$, 229 (62), 214 (23), 198 (41), 186 (24), 170 (47), 157 (25), 133 (18). HRMS (ESI): calcd for $[\mathrm{M}+\mathrm{Na}]^{+}:$253.0635; found: 253.0621 .

\section{Literature}

[1] Strekowski, L.; Cegla, M. T.; Harden, D. B.; Kong, S.-B. J. Org. Chem. 1989, 54, 2464.

[2] Ōkubo, M.; Ueda, S. Bull. Chem. Soc. Jpn. 1979, 52, 3346.

[3] Oi, S.; Fukita, S.; Hirata, N.; Watanuki, N.; Miyano, S,; Inoue, Y. Org. Lett. 2001, 3,2579 . 


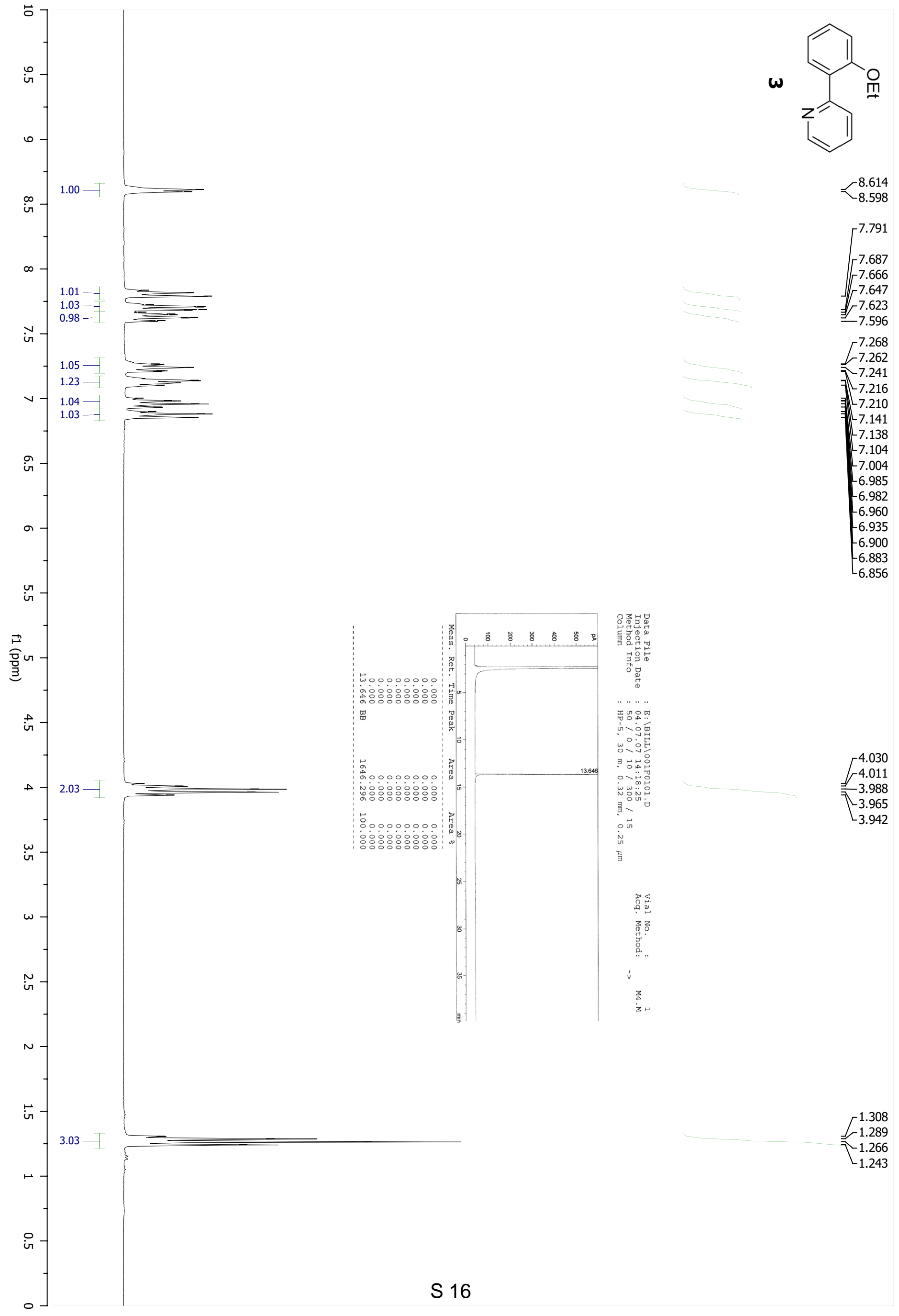




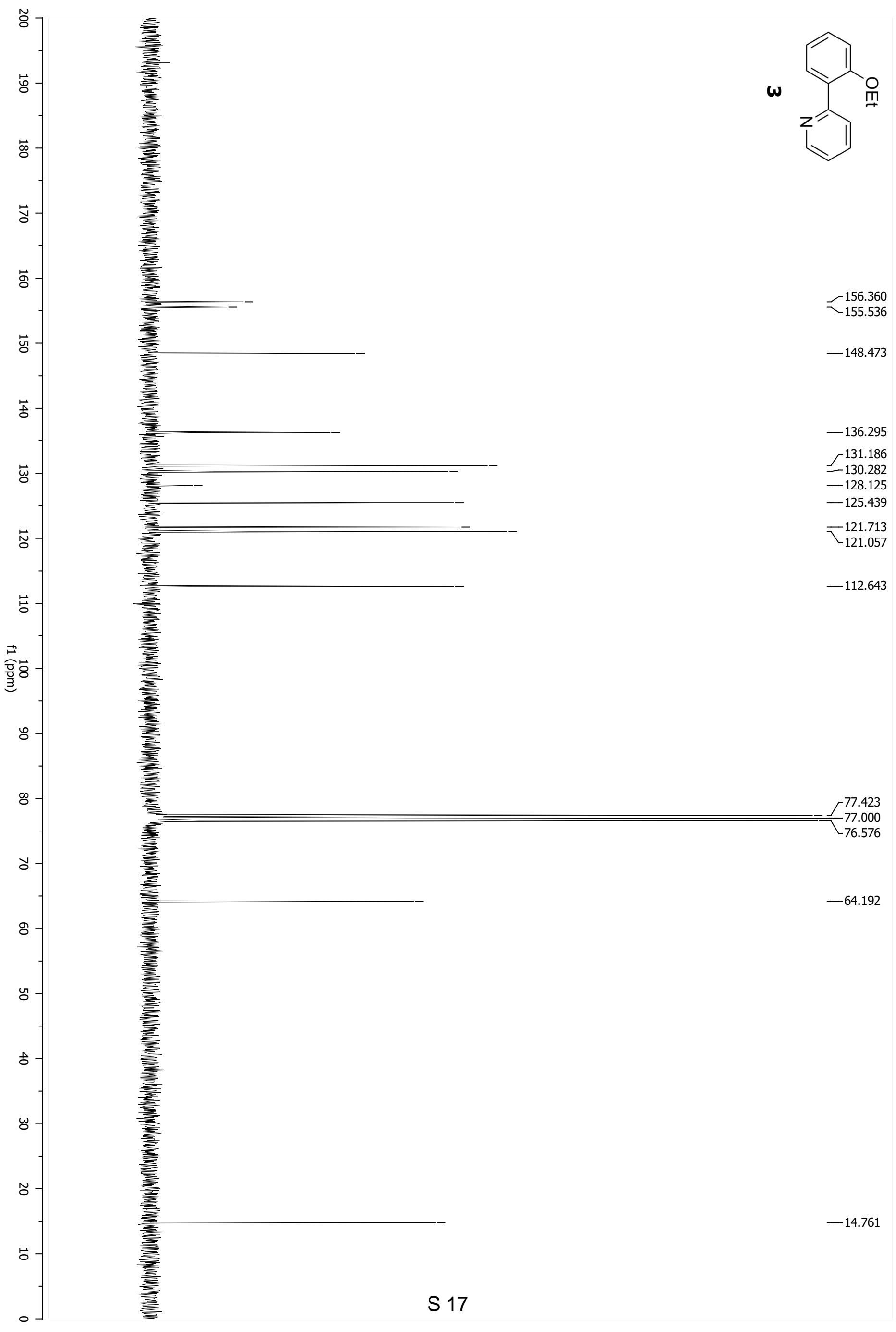




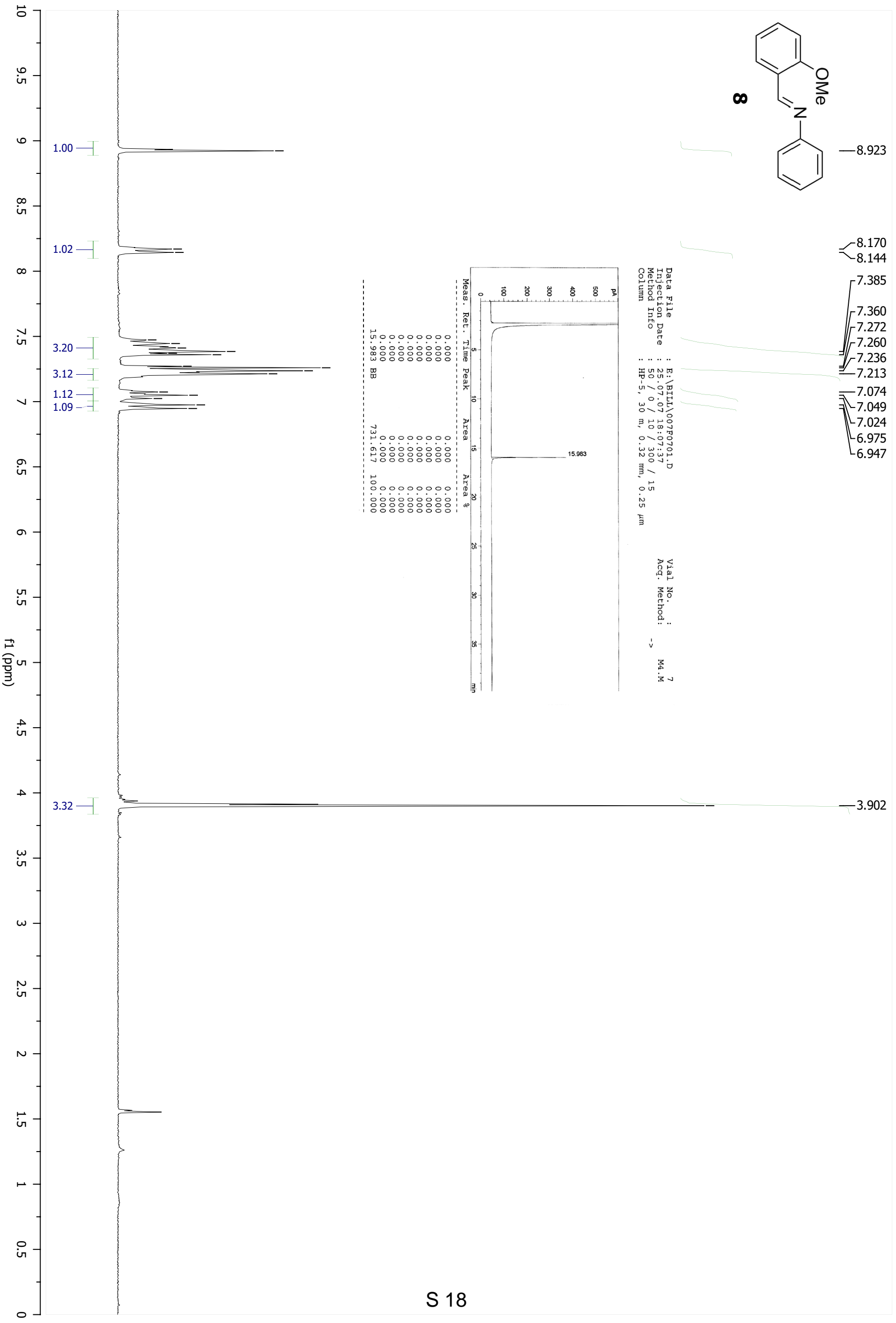




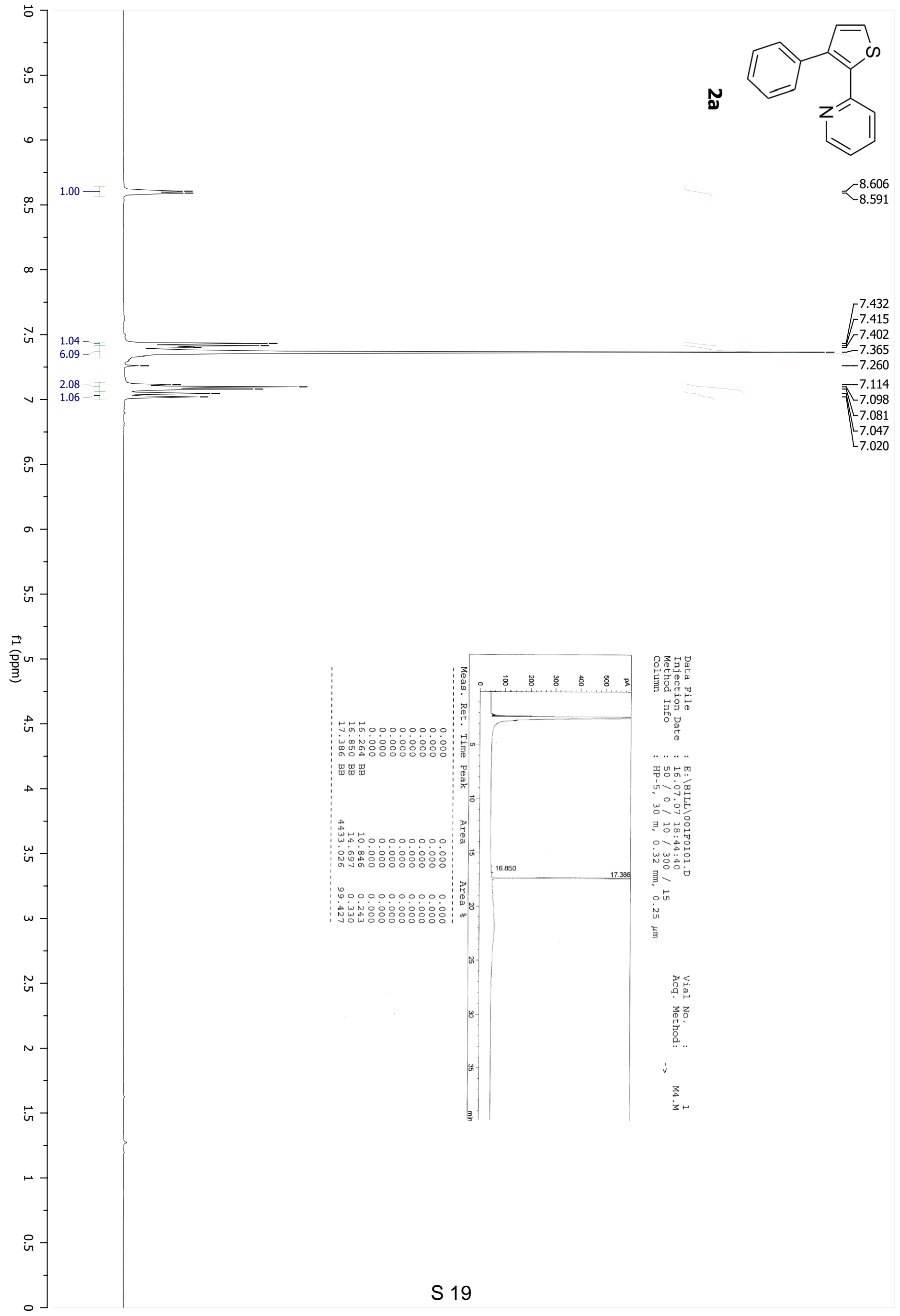




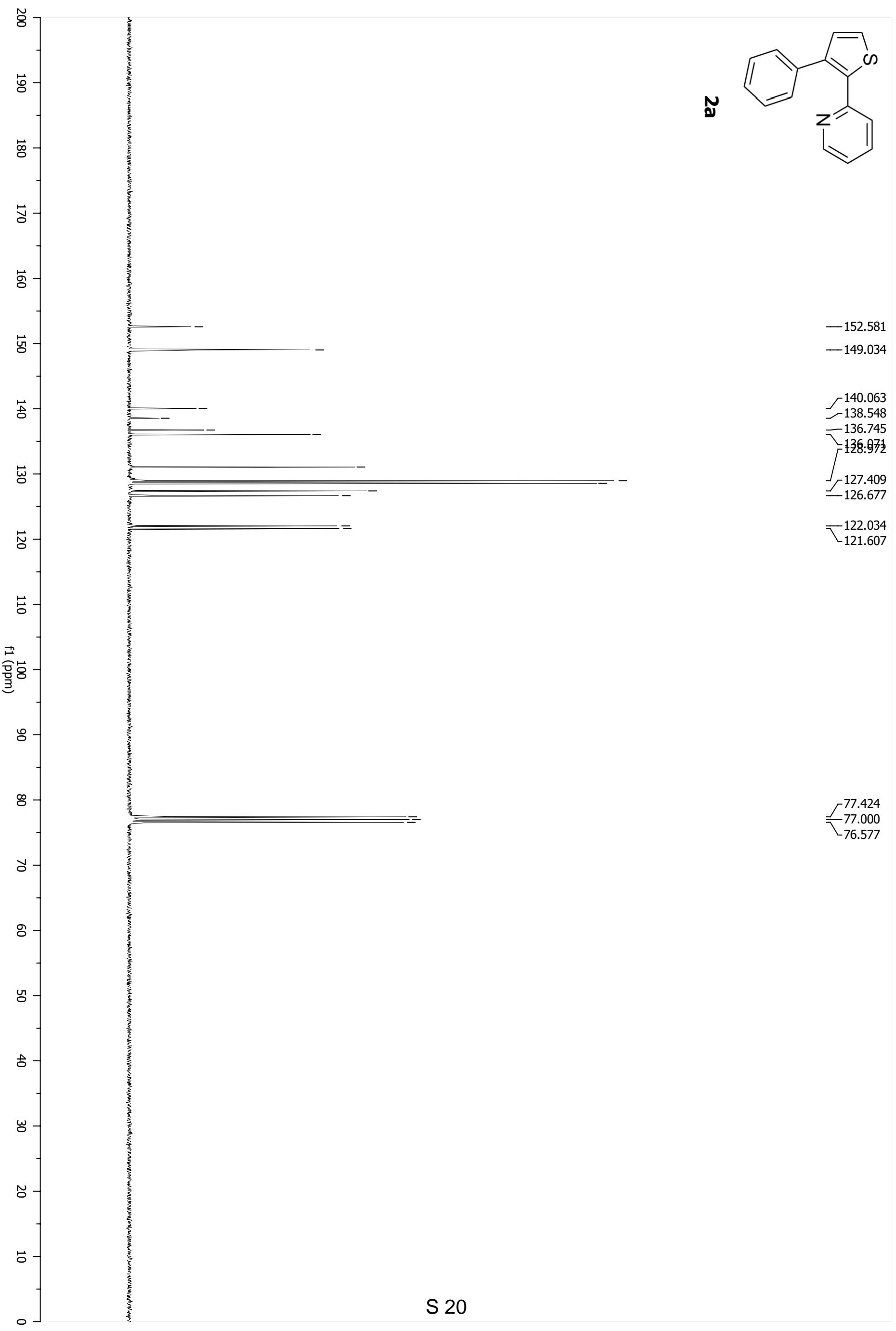




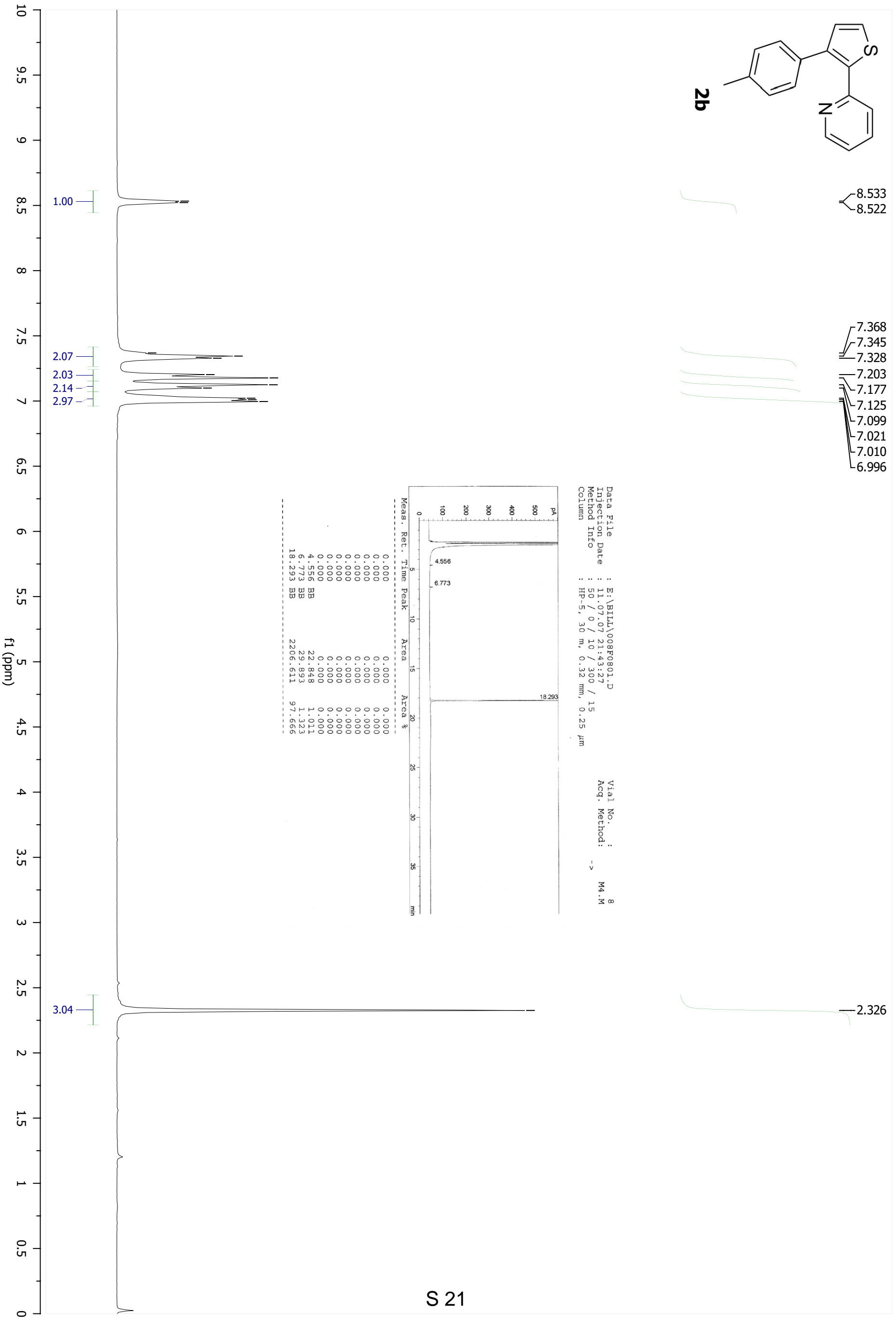




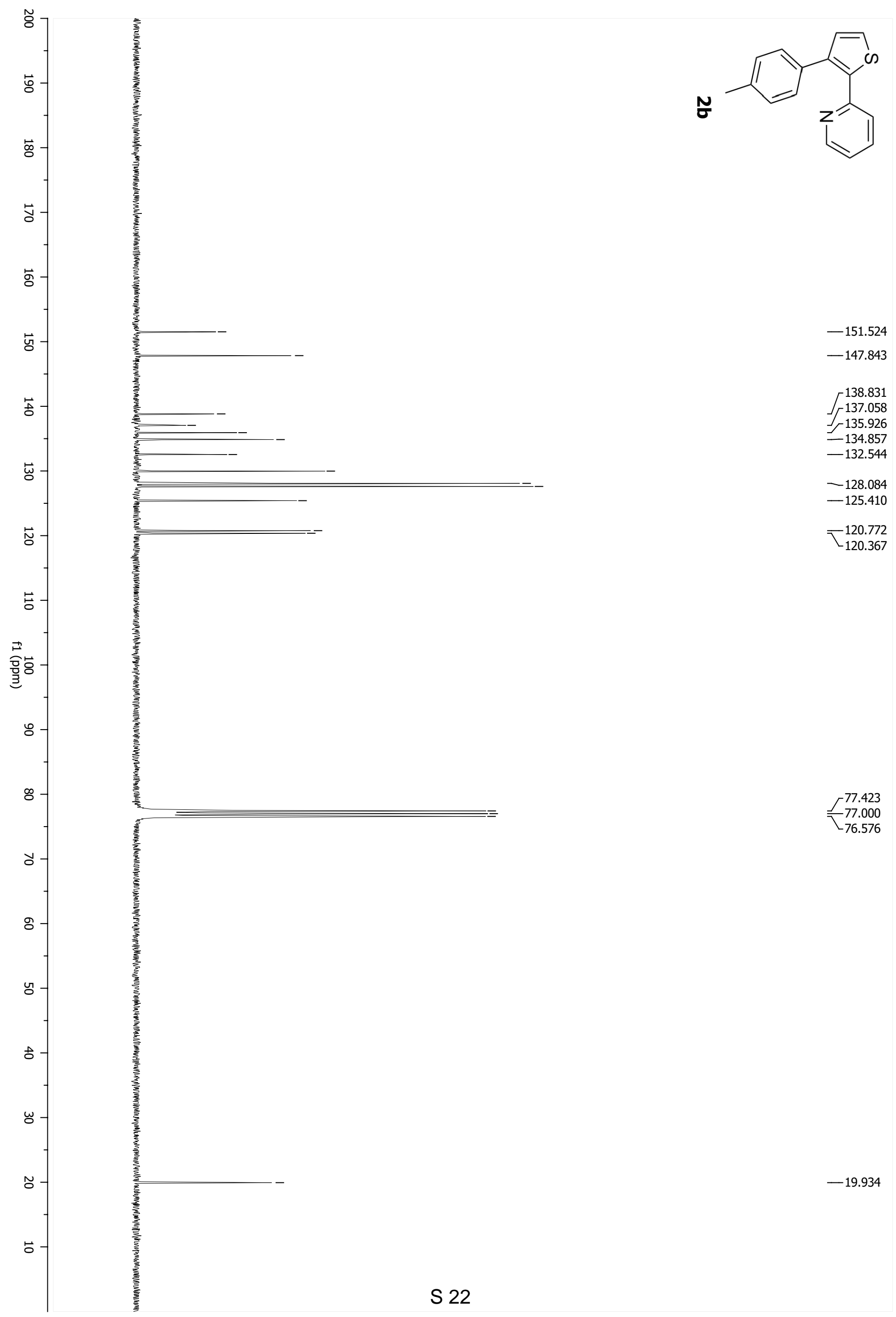




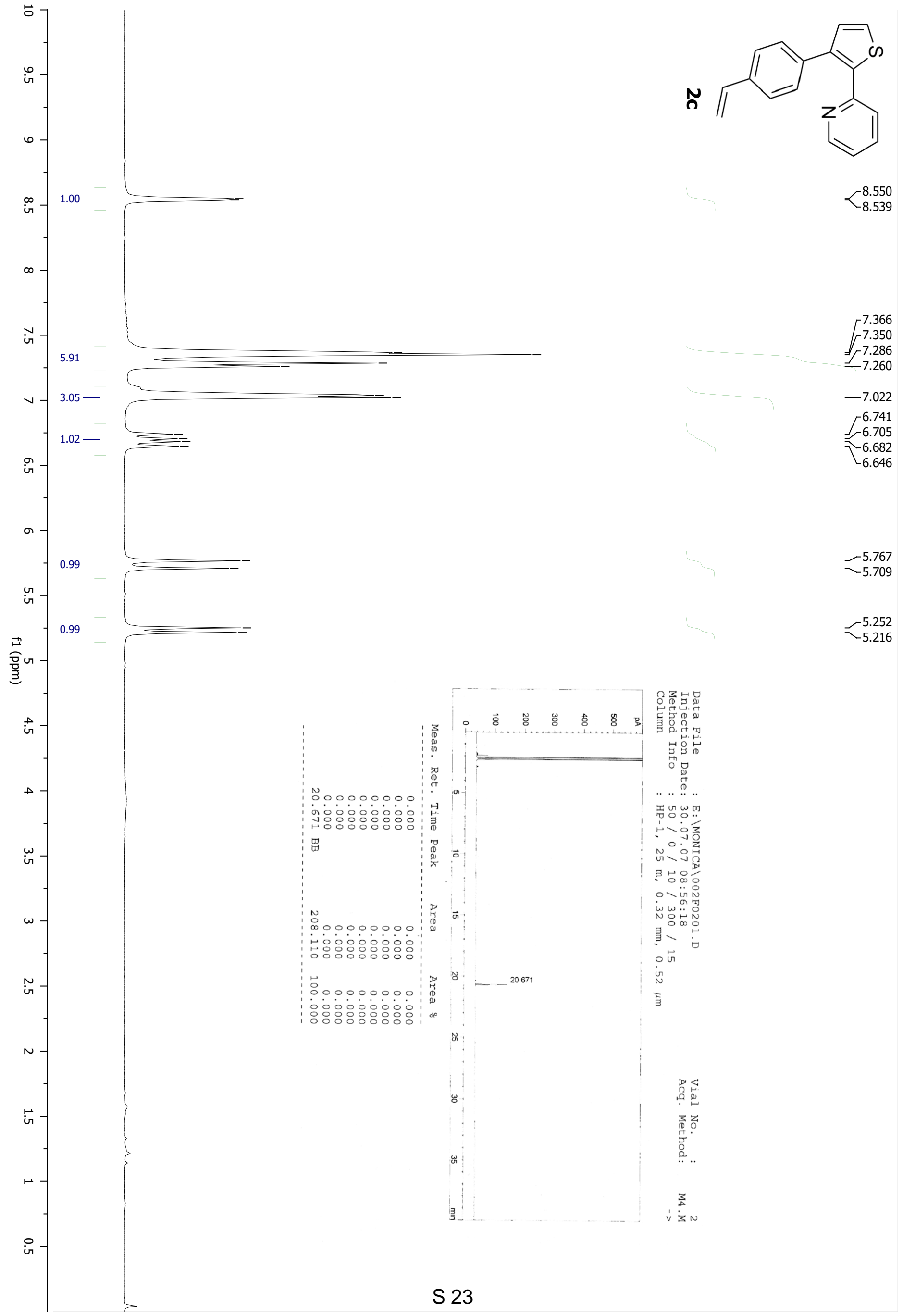




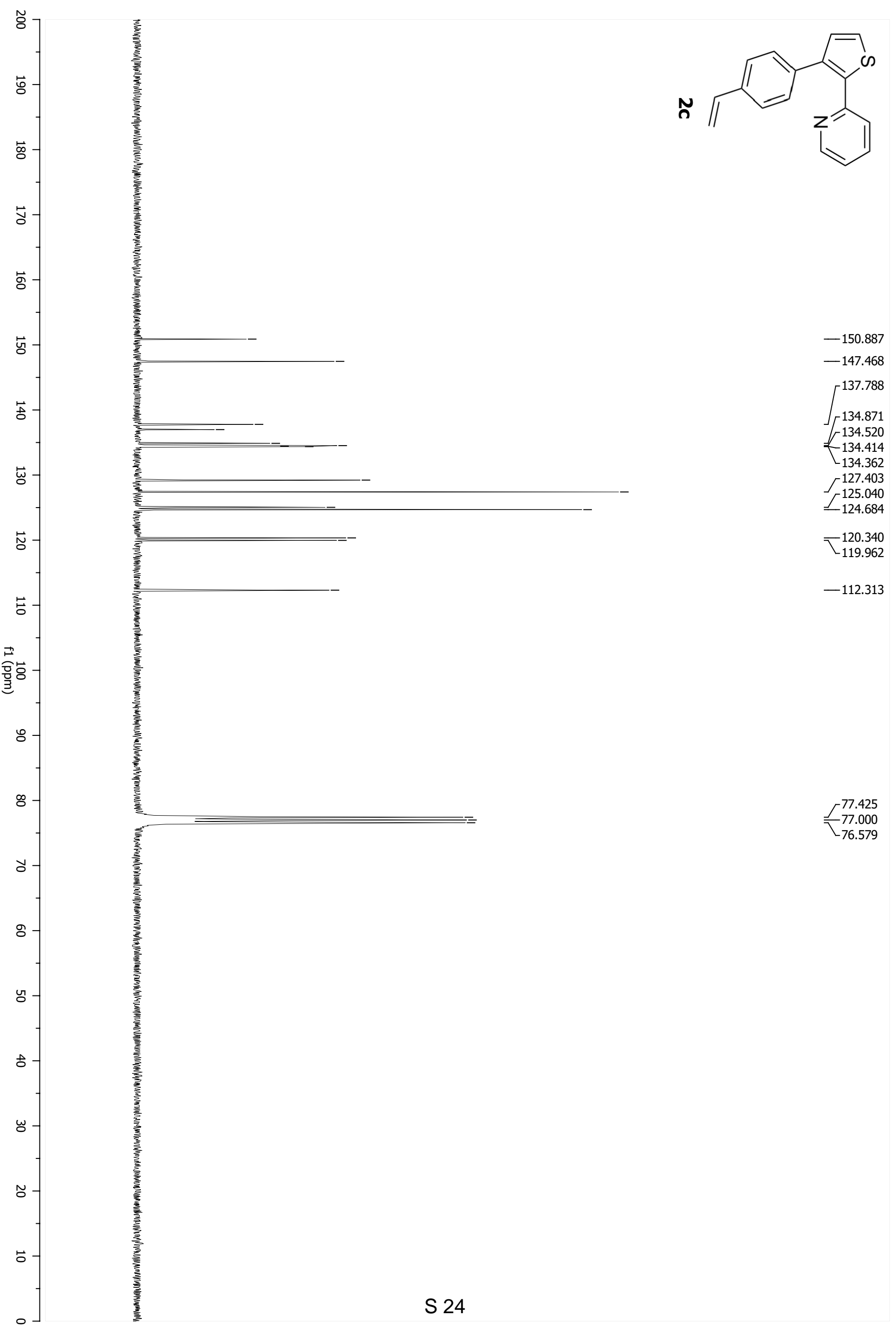




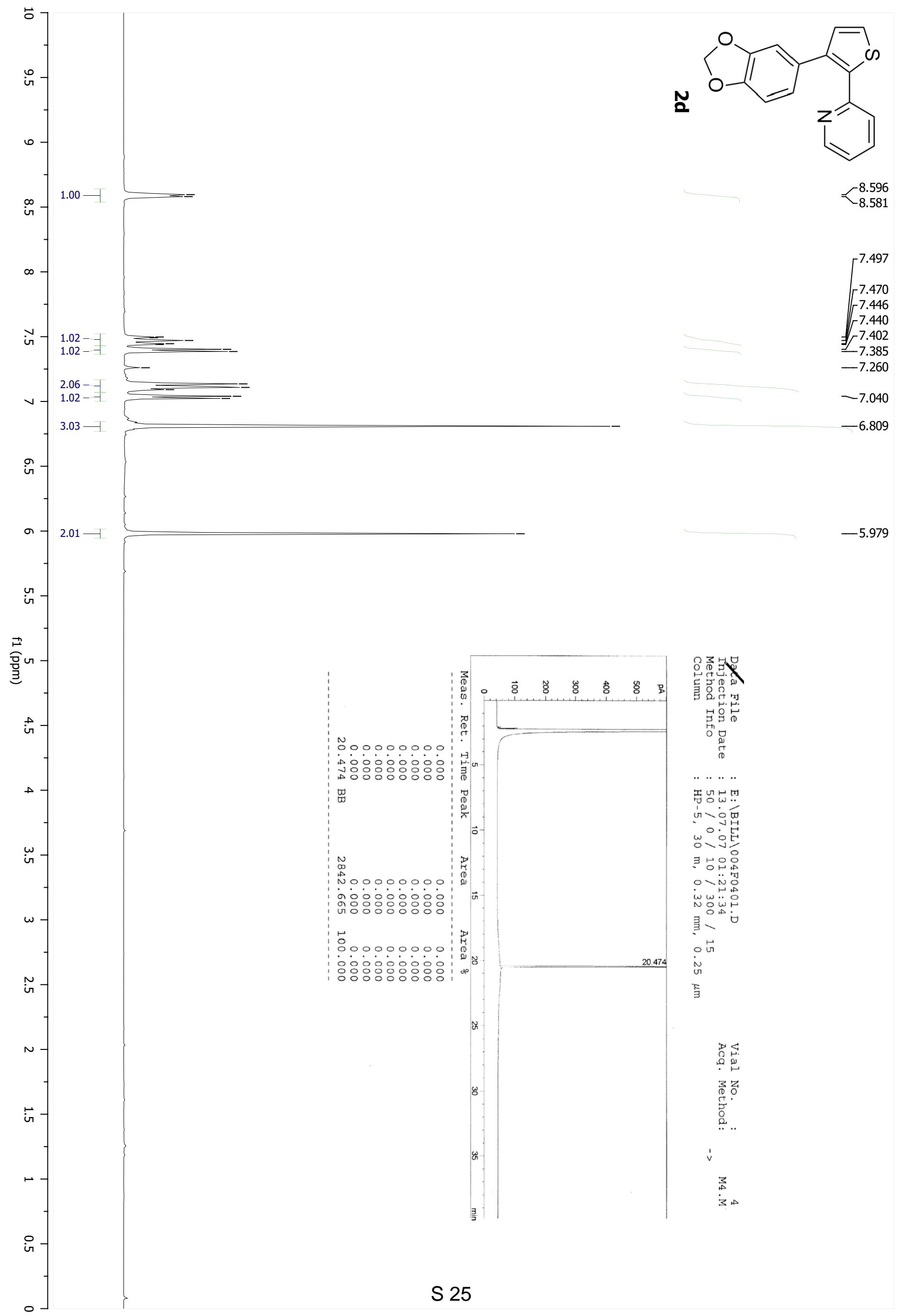




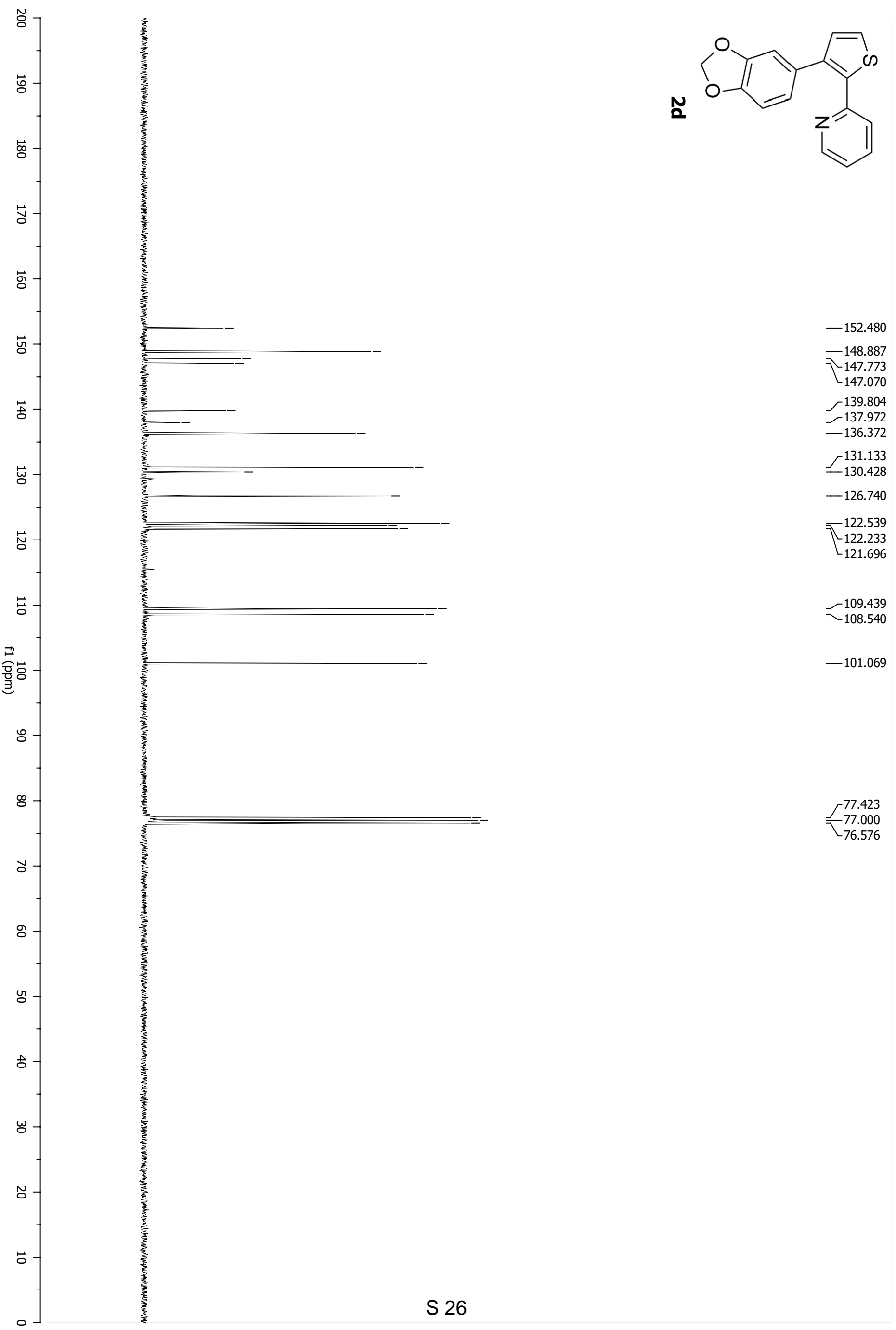




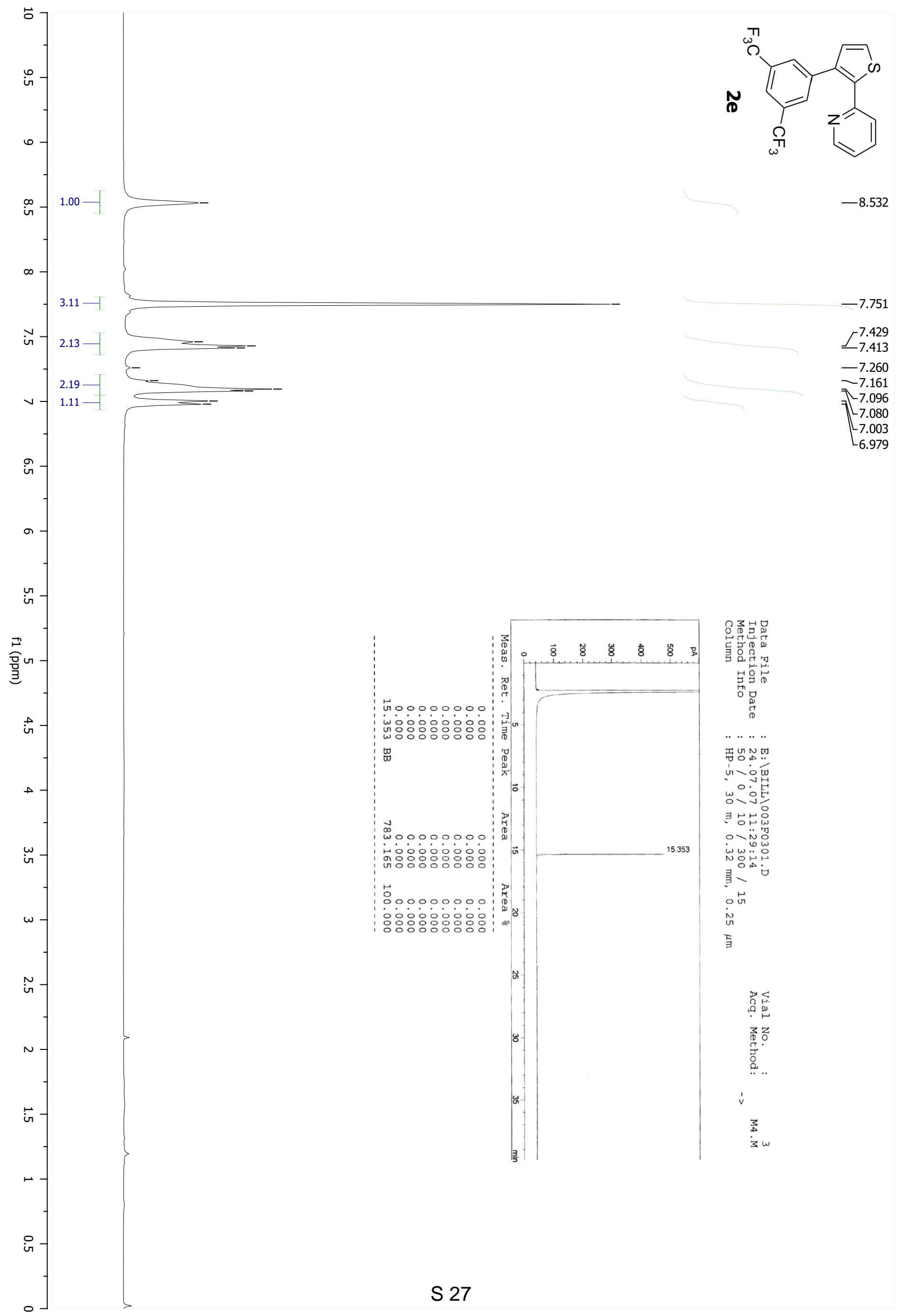




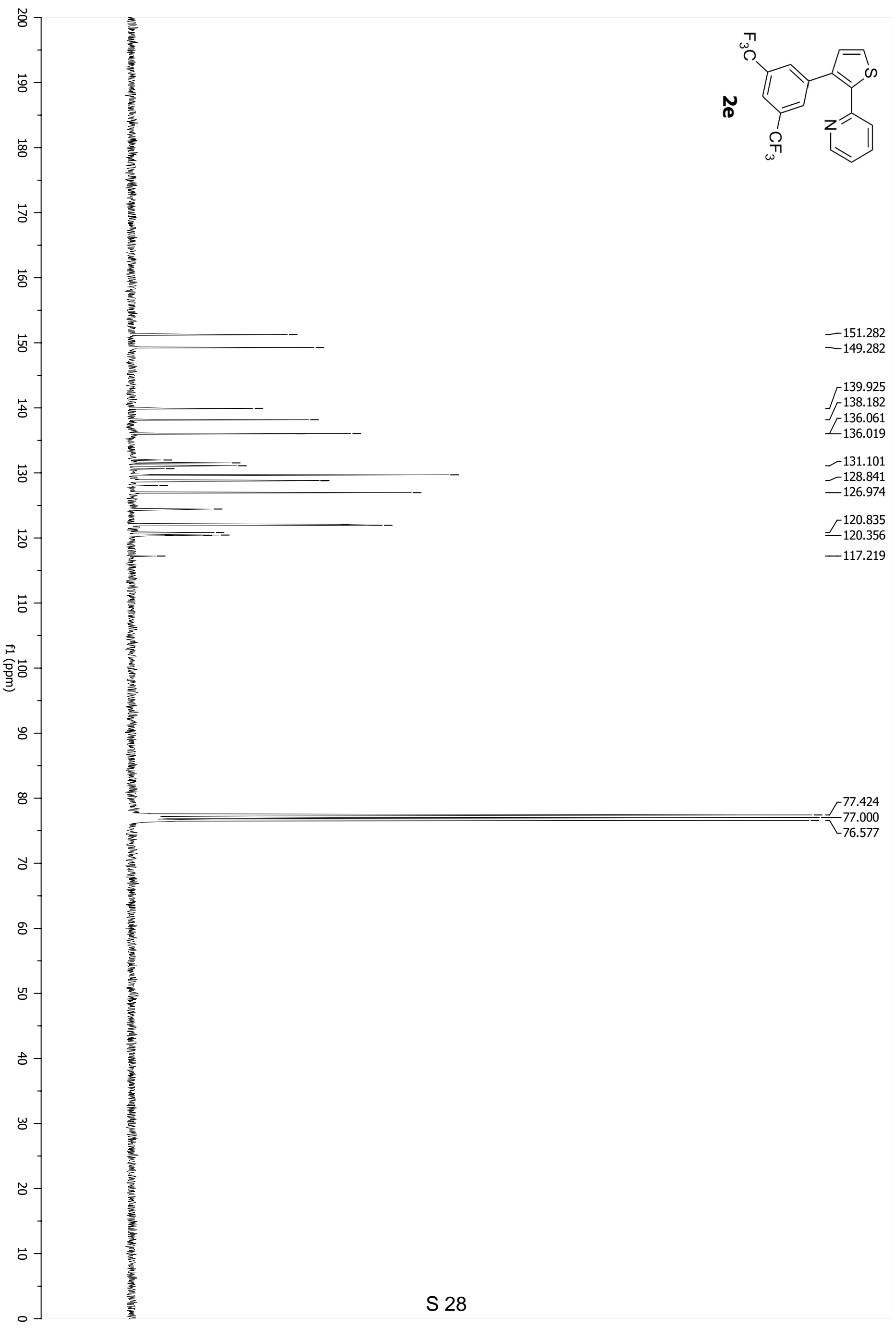




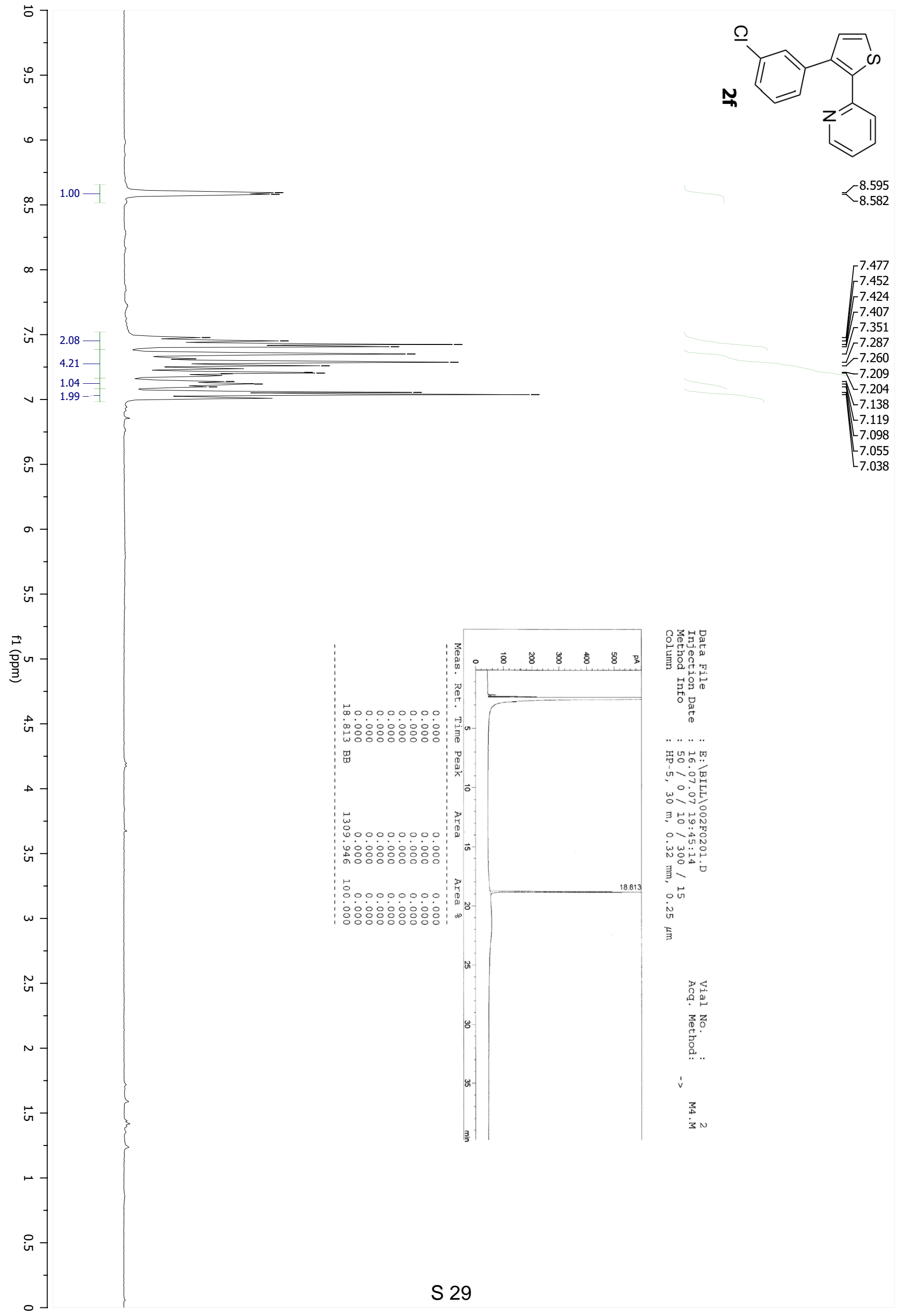




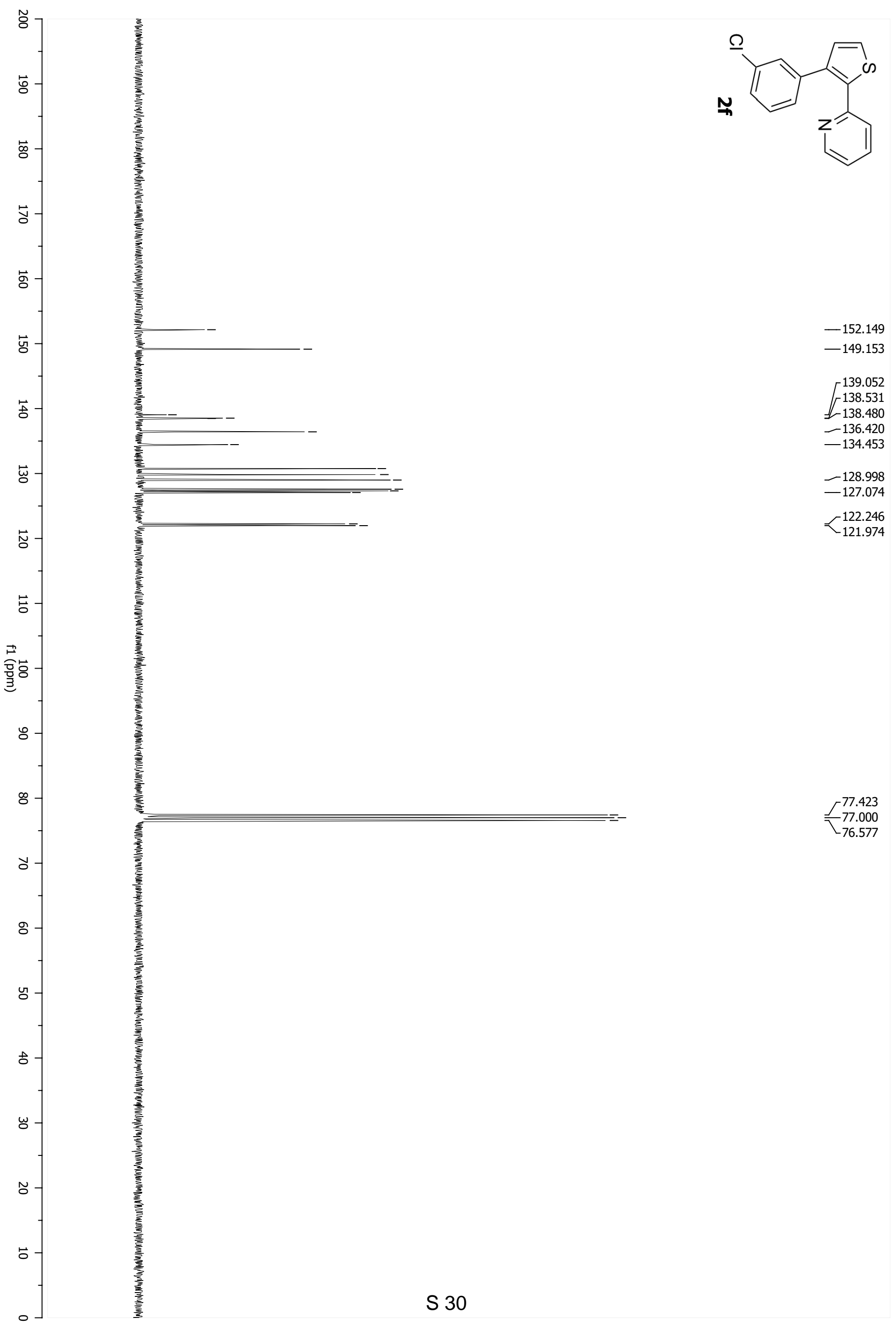




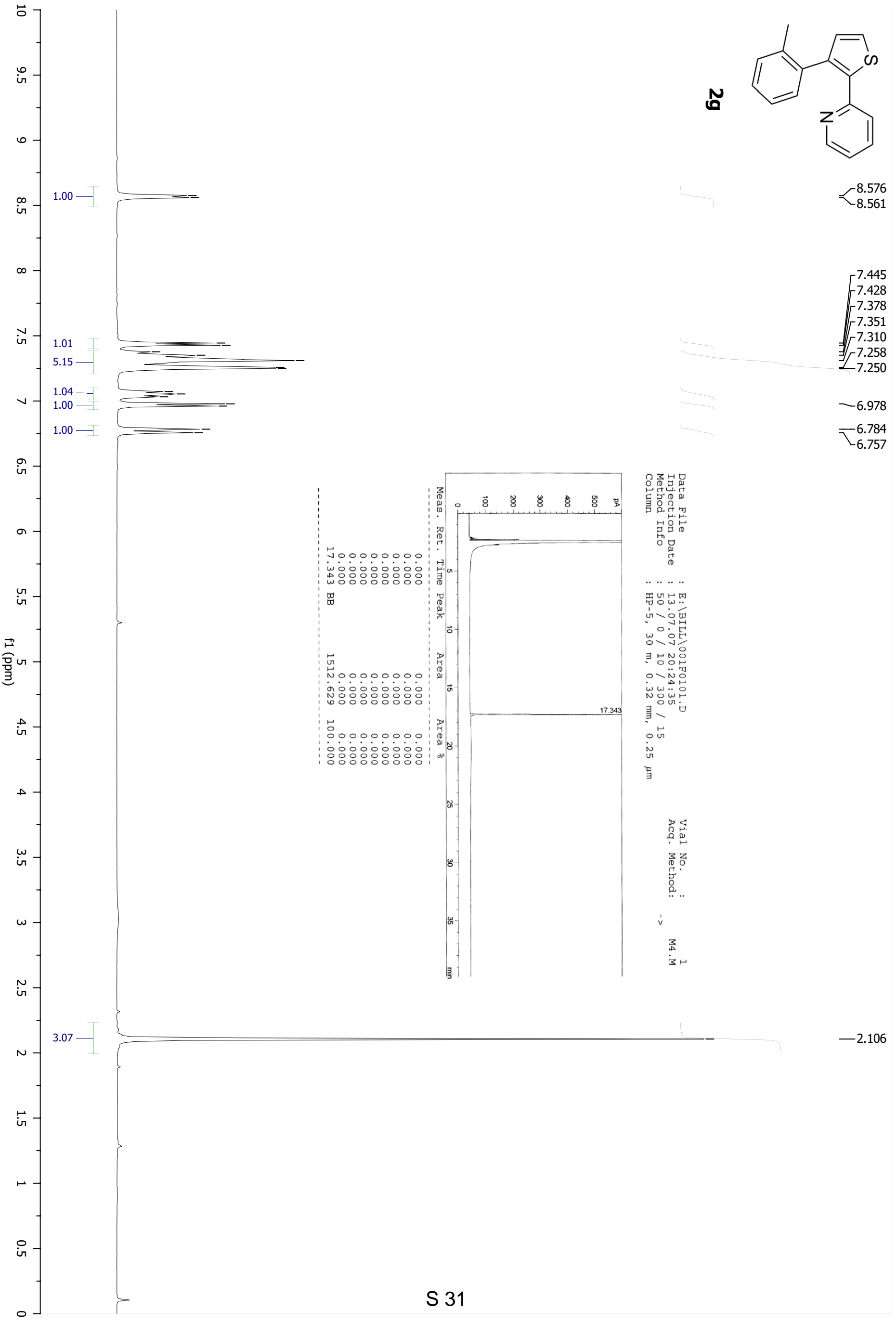




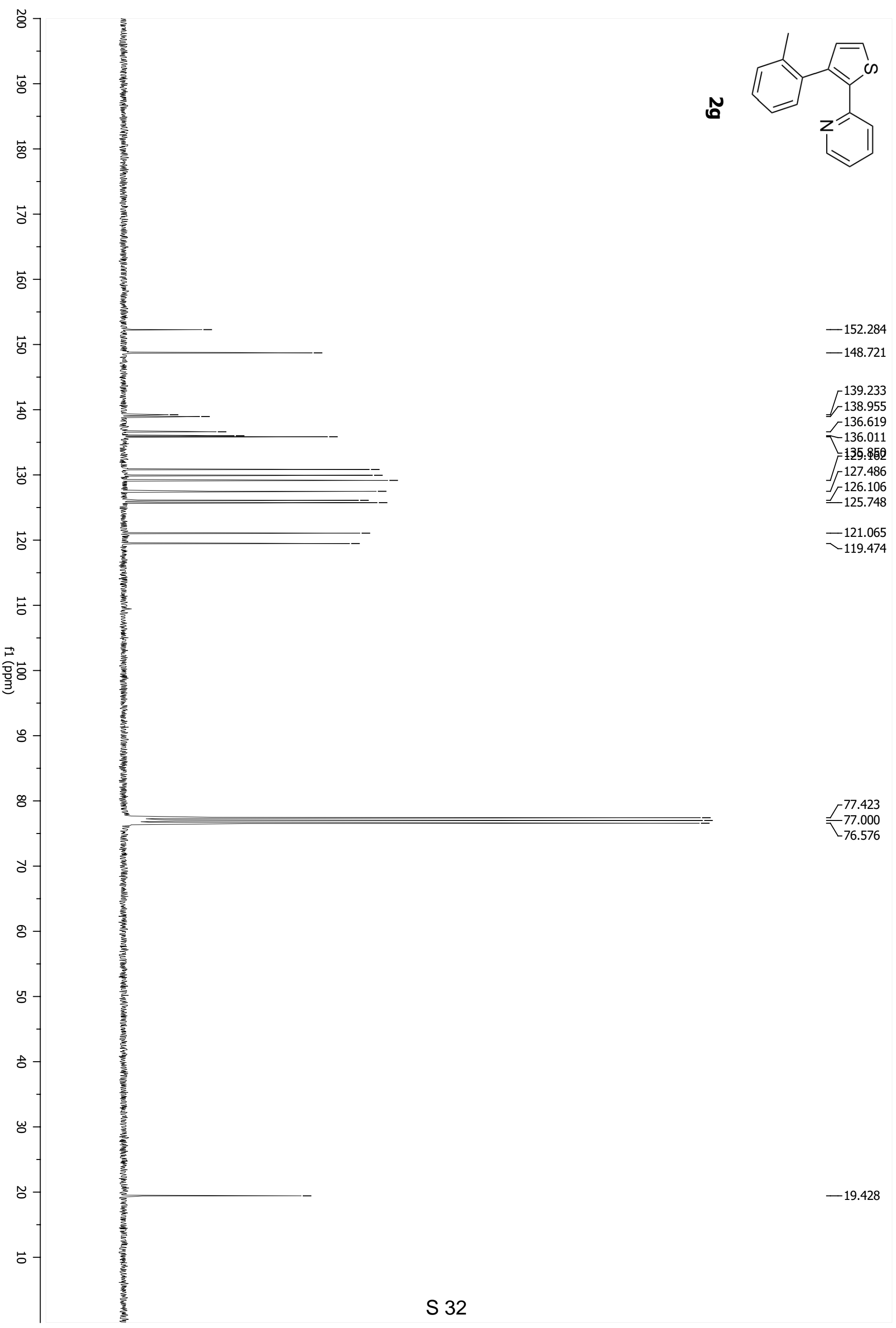




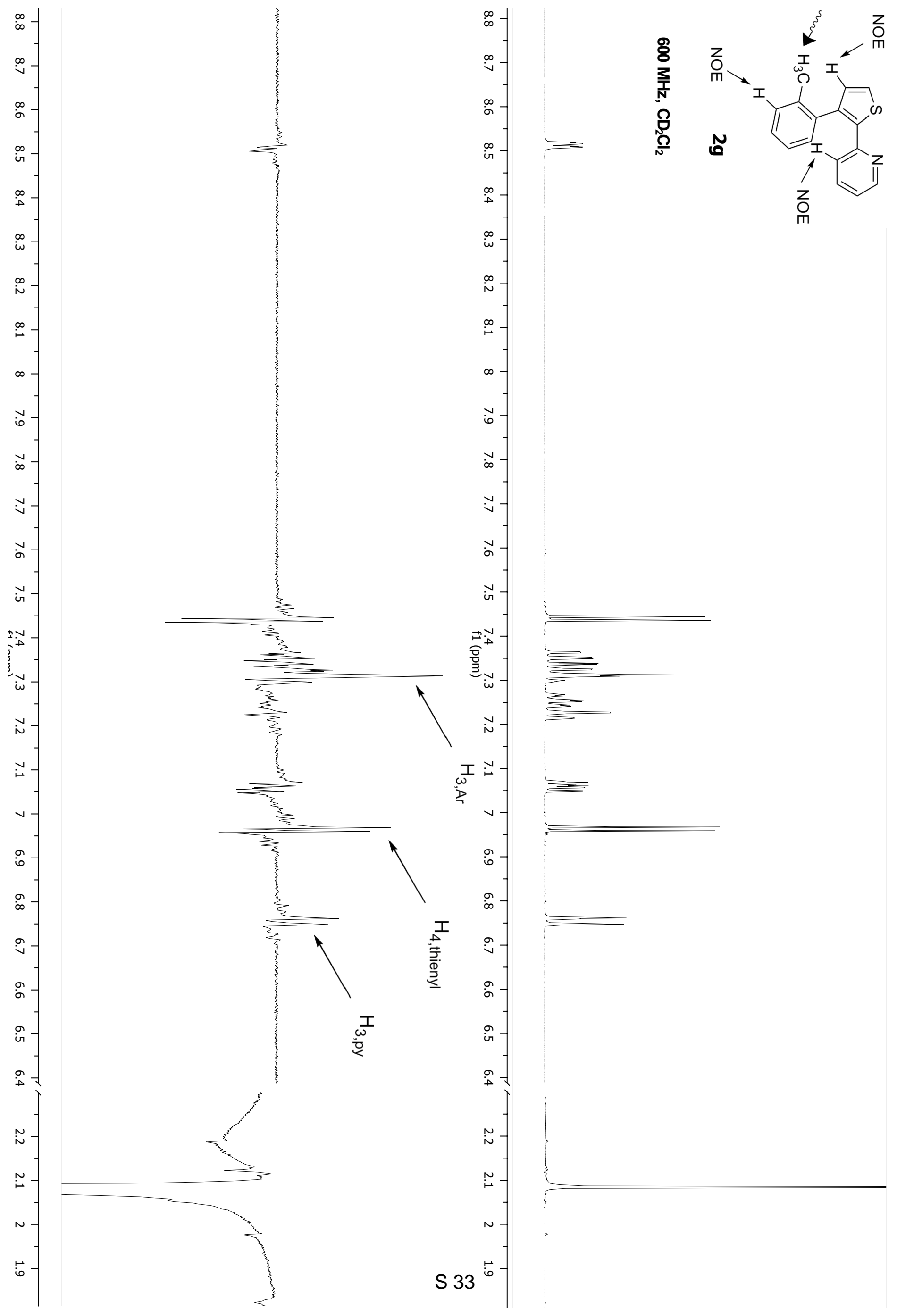




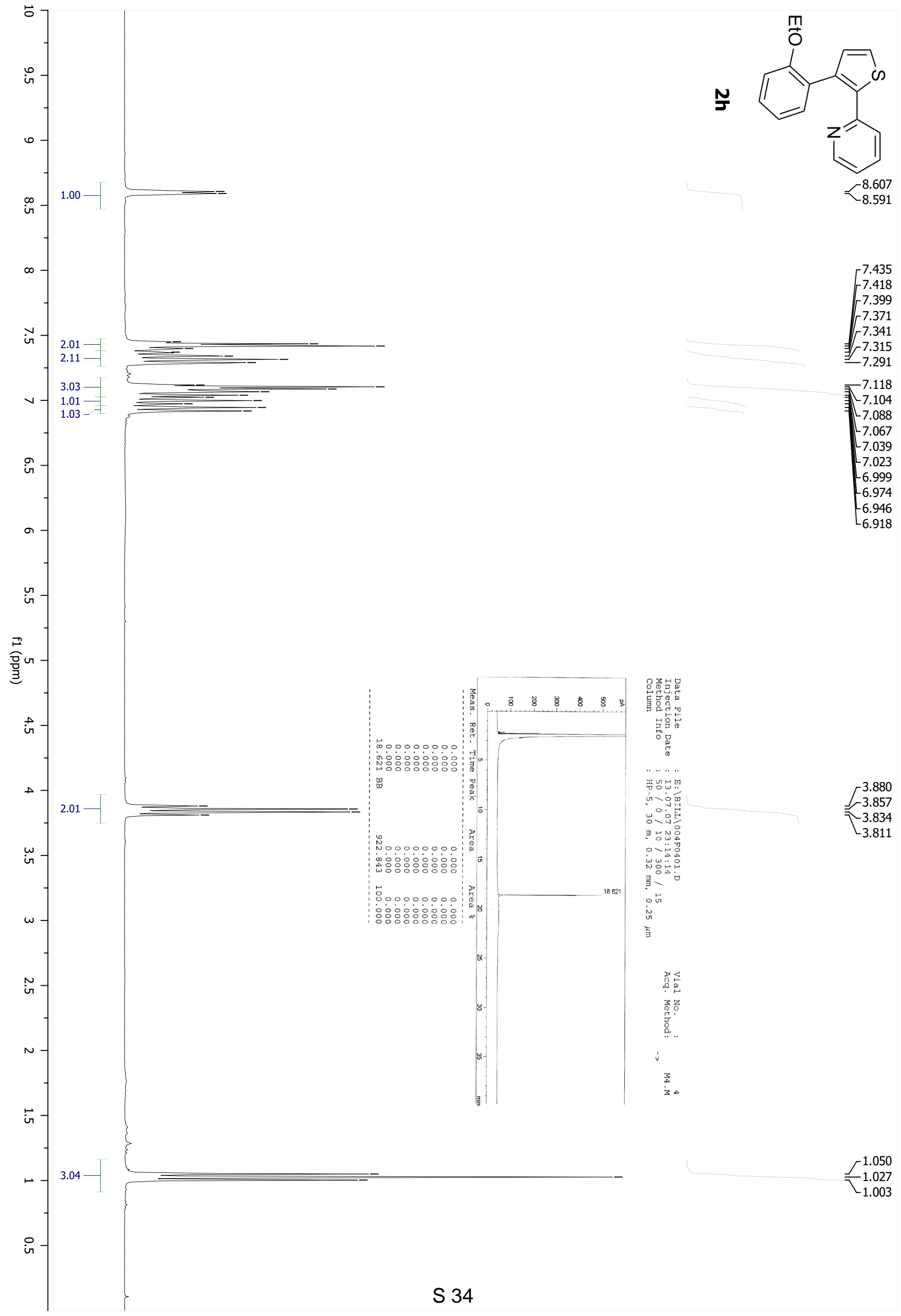




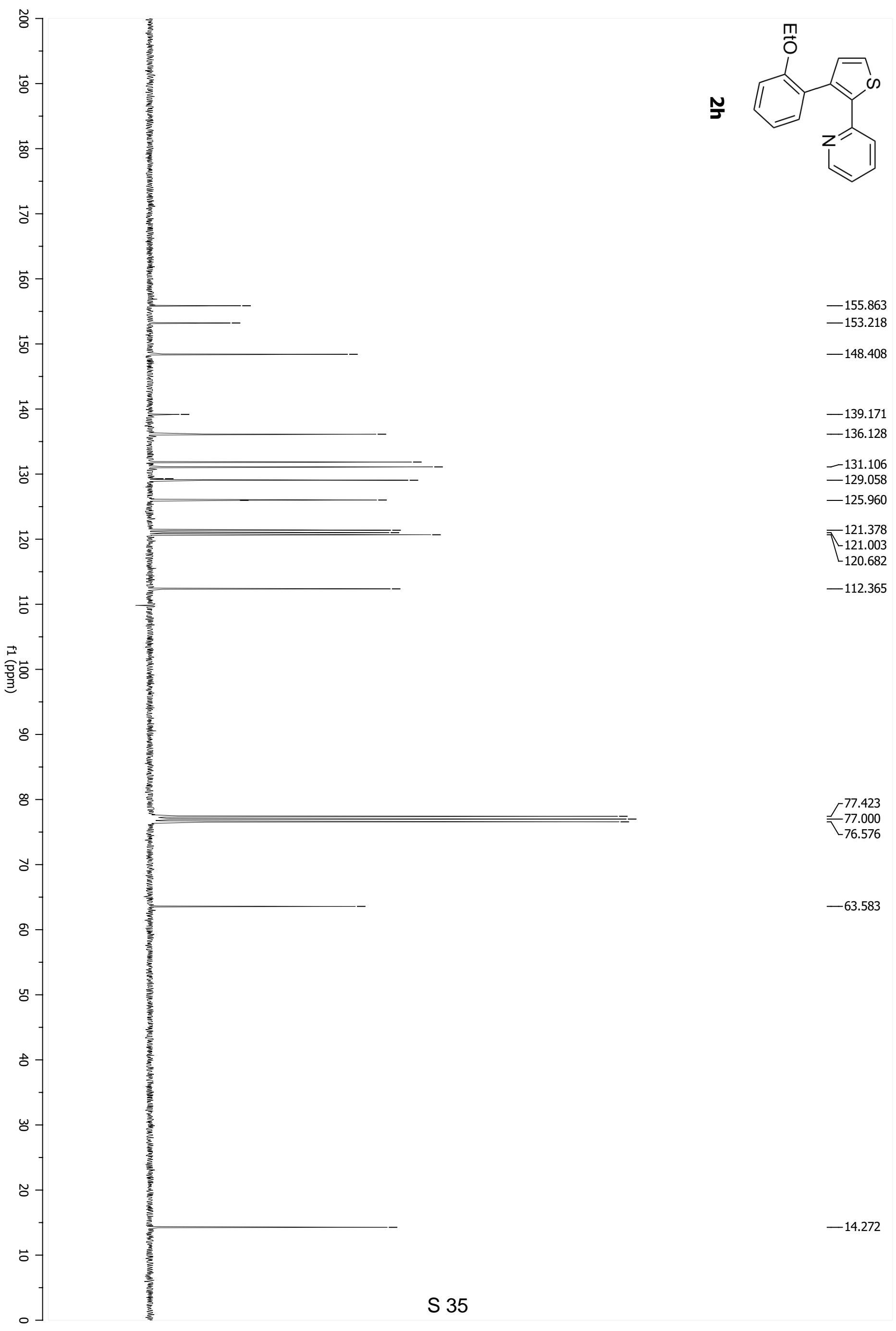




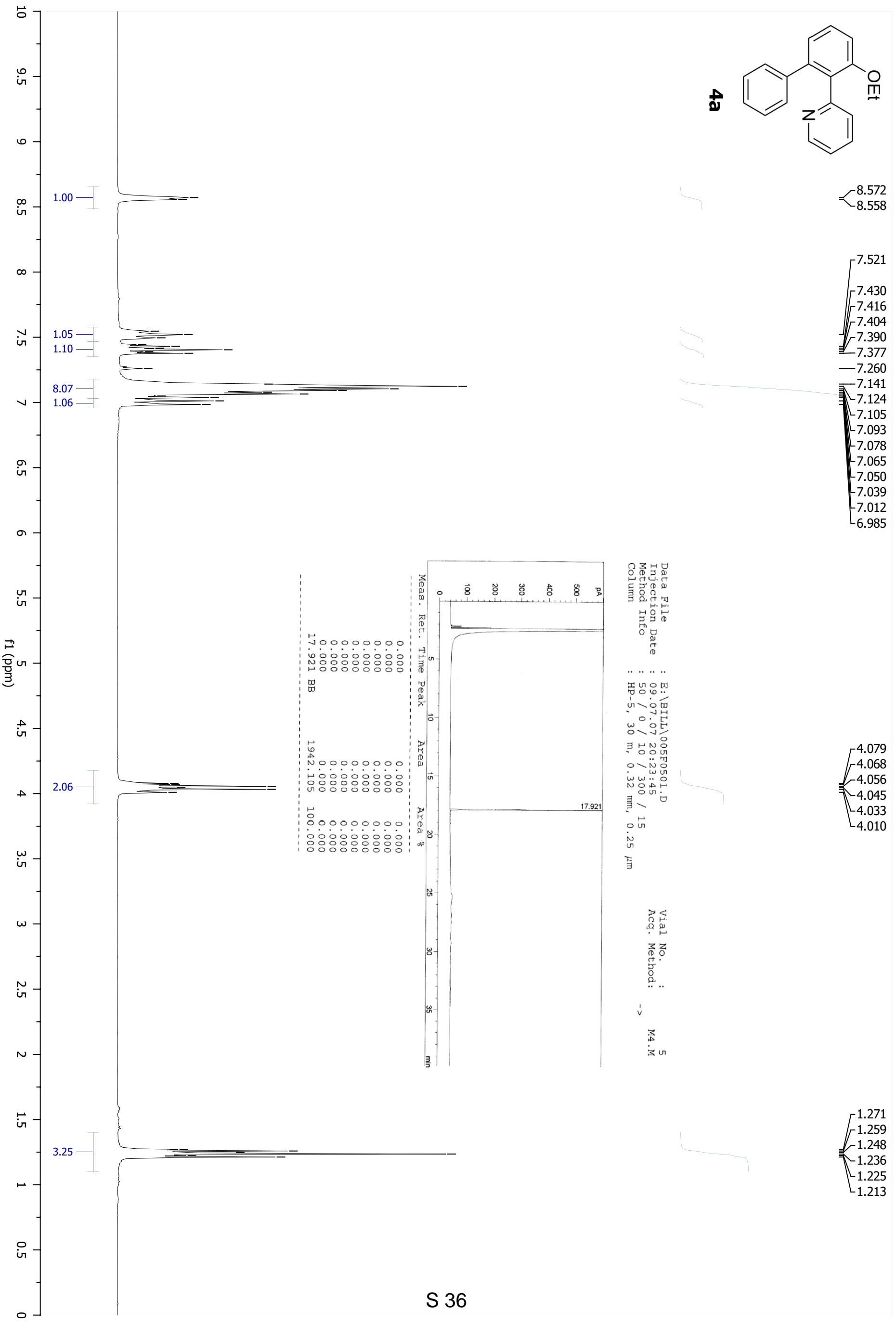




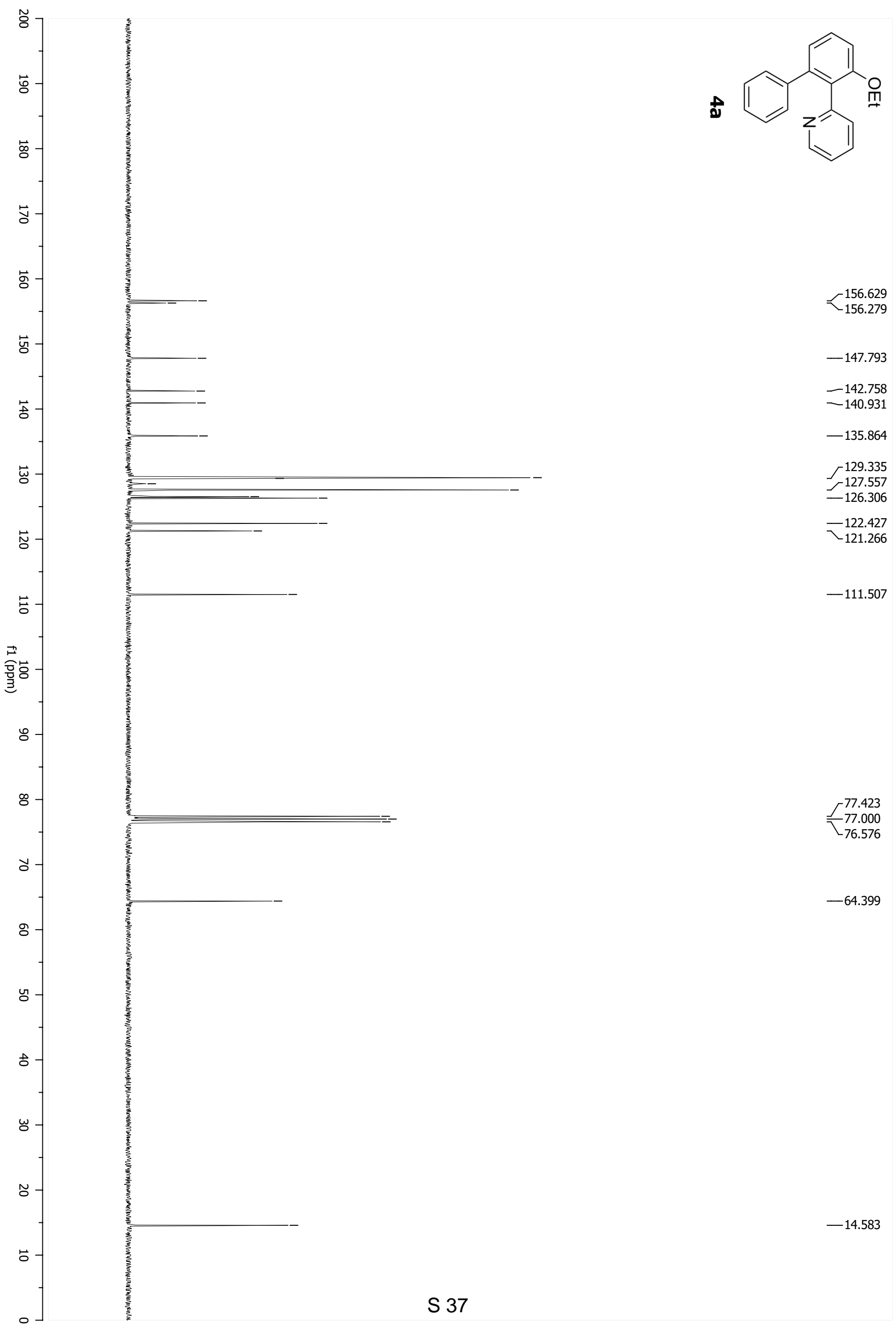




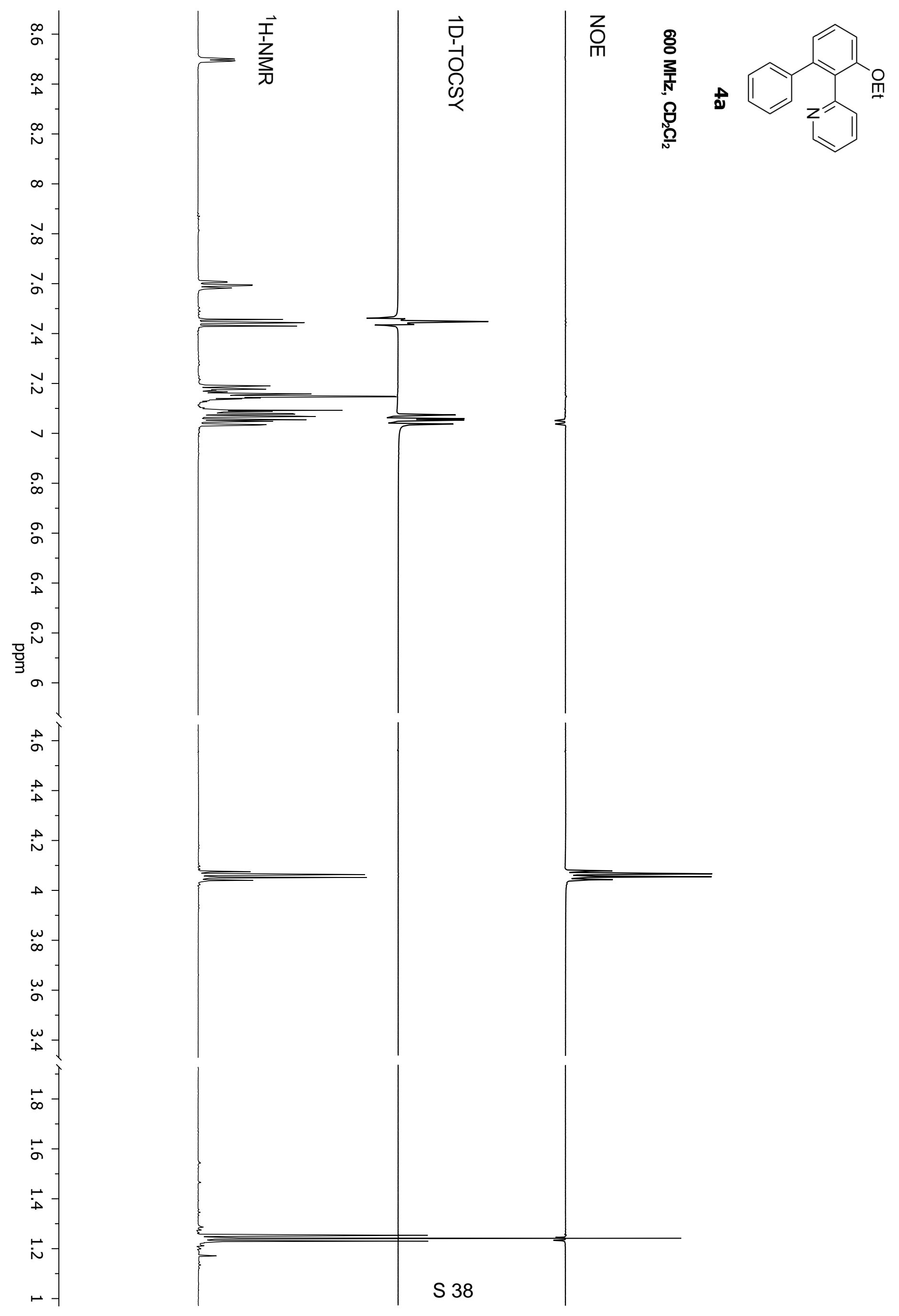




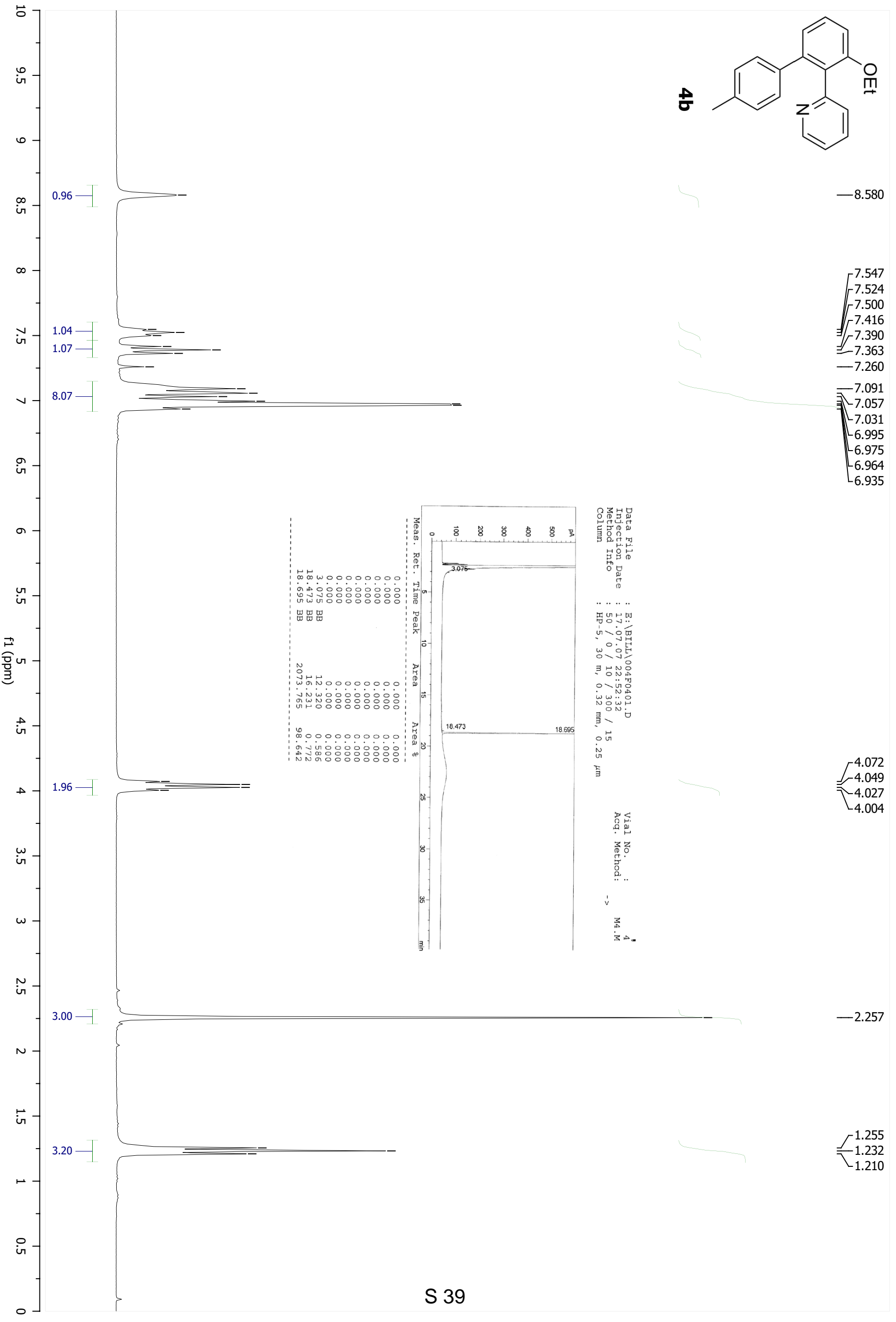




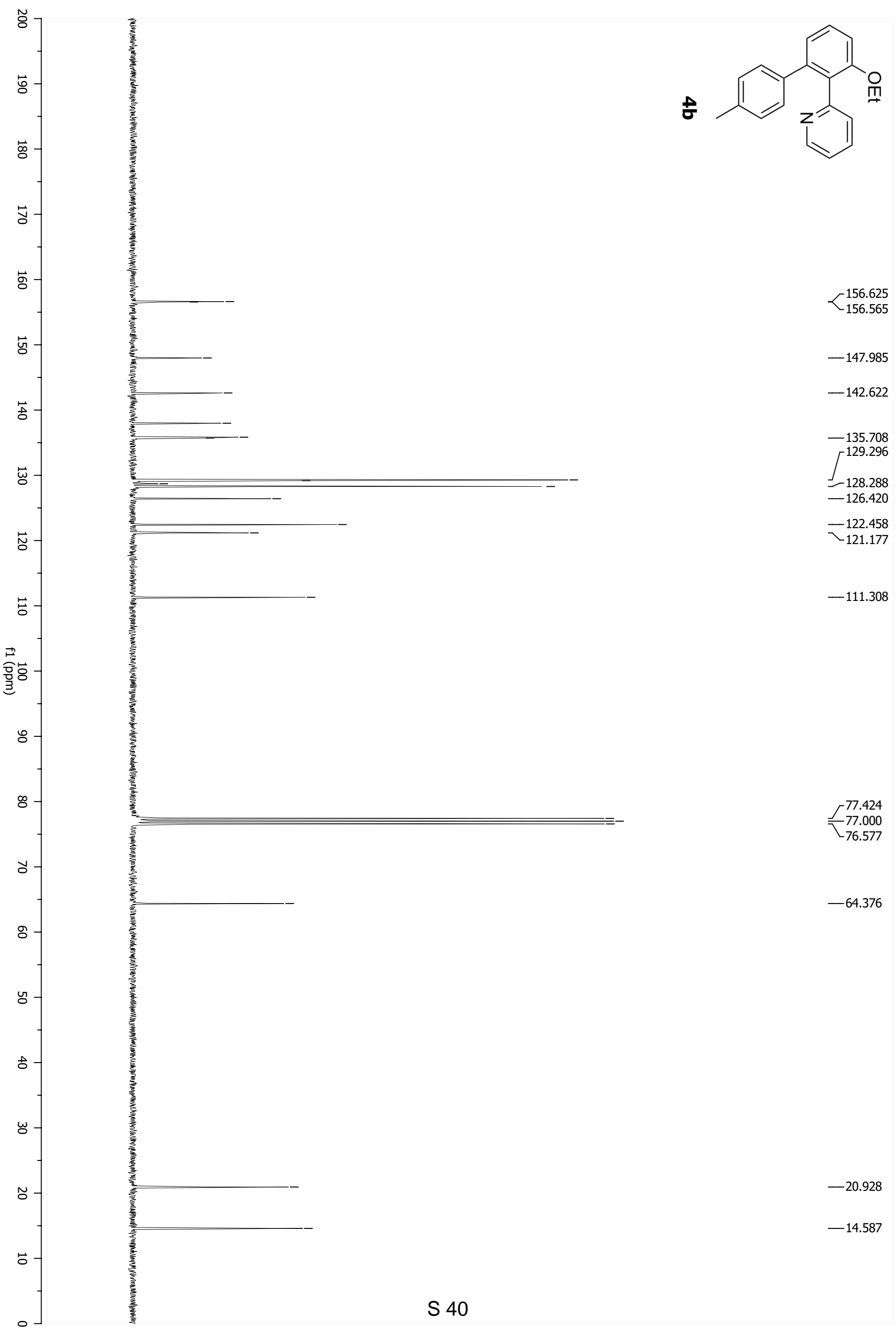




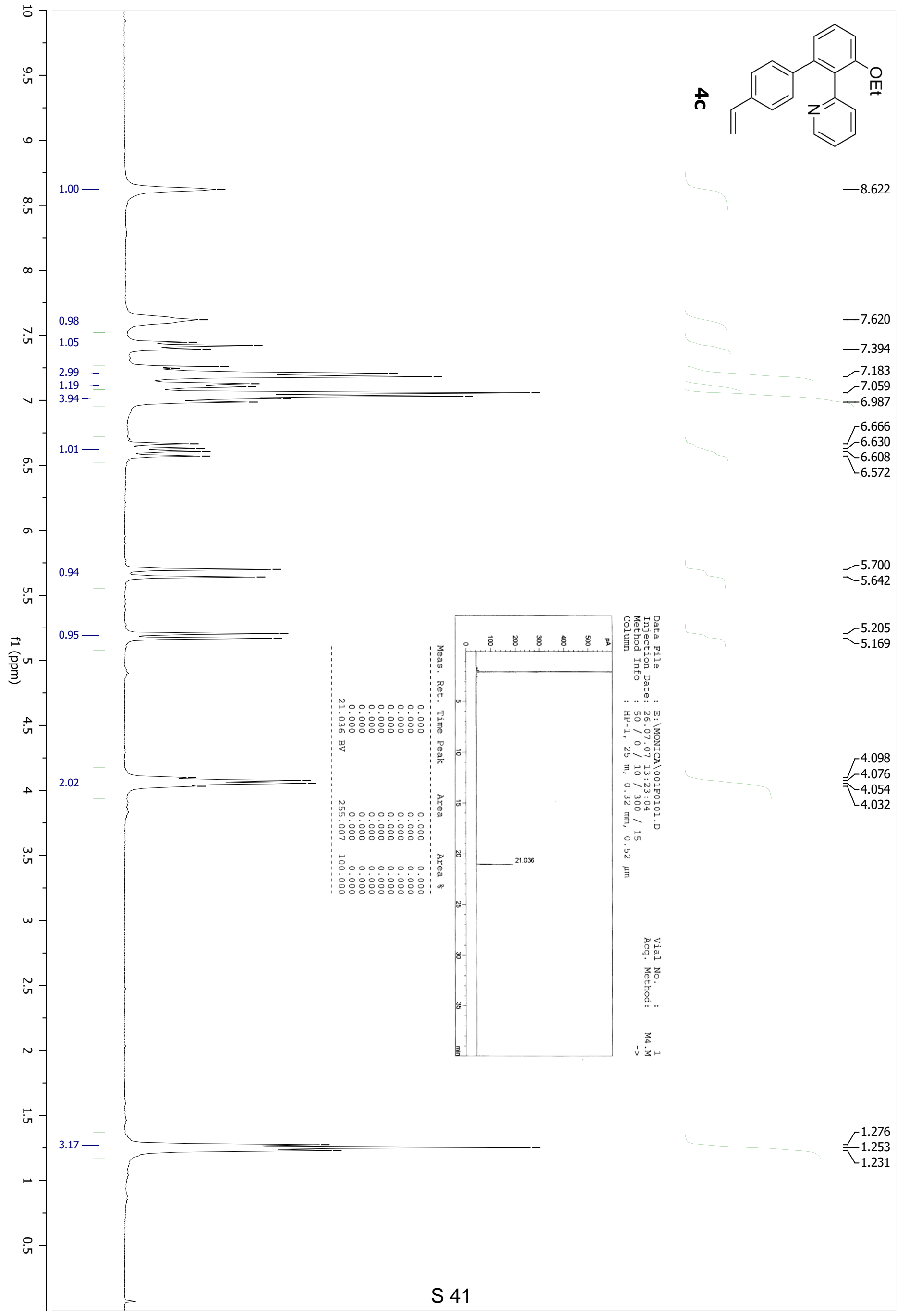




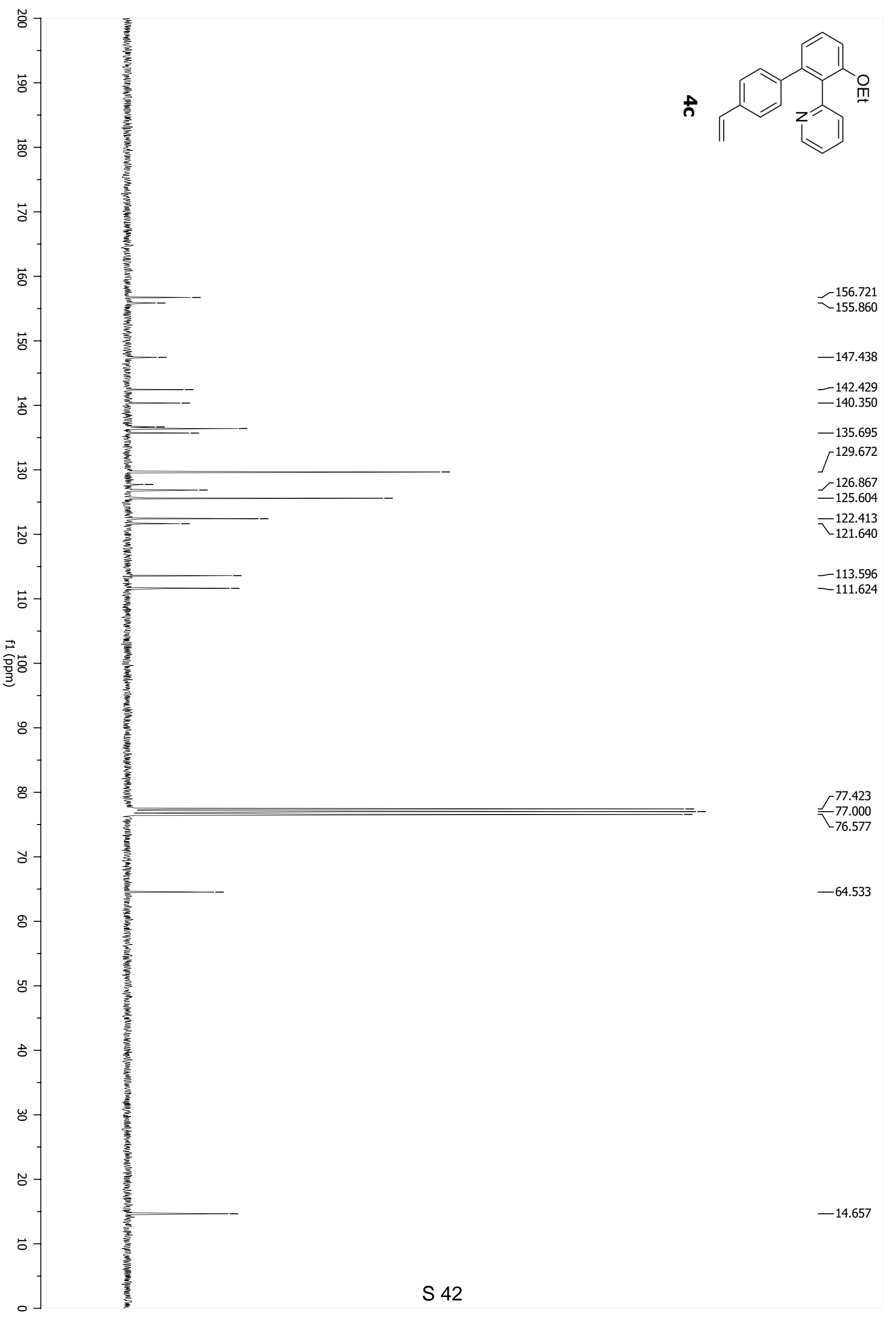




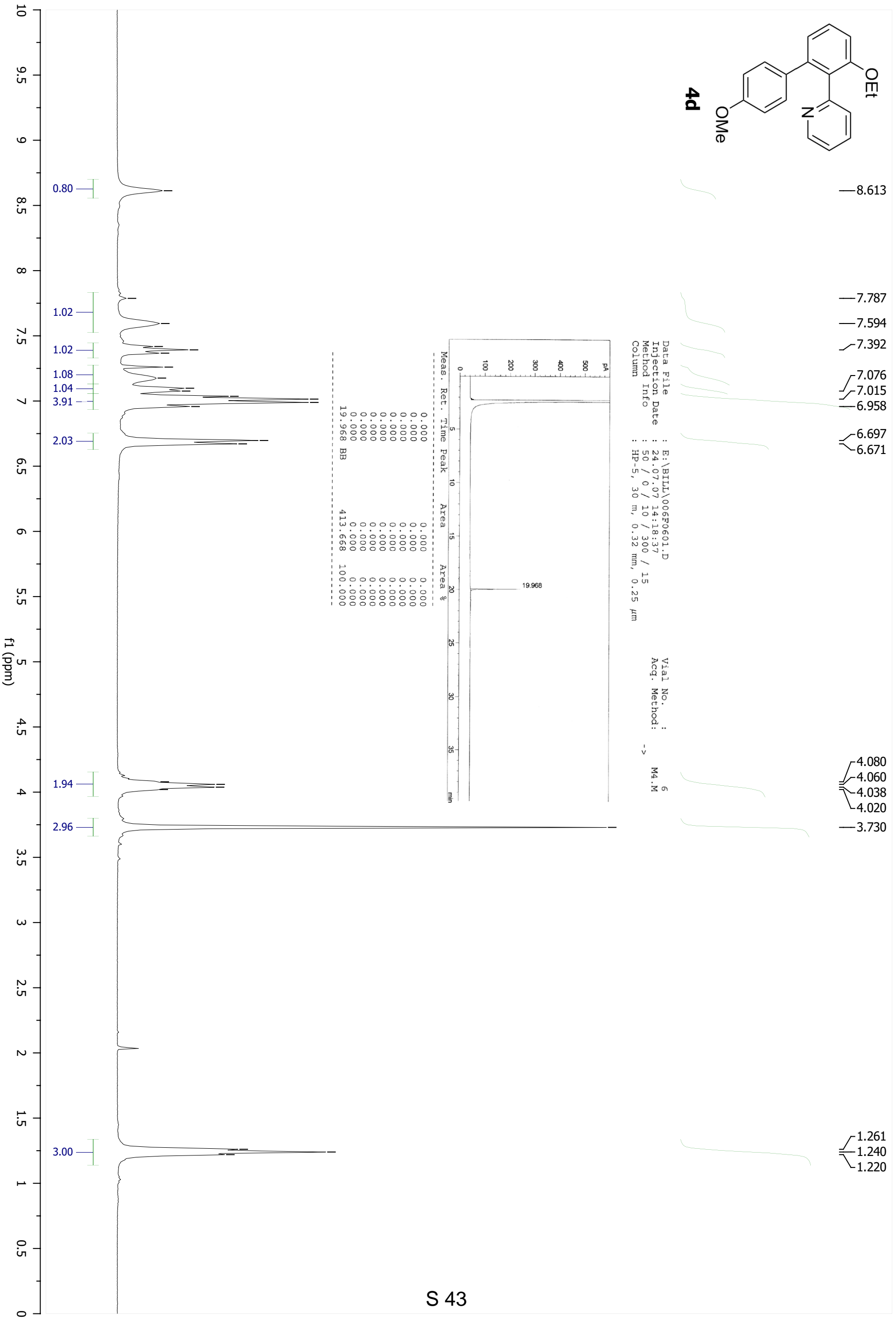




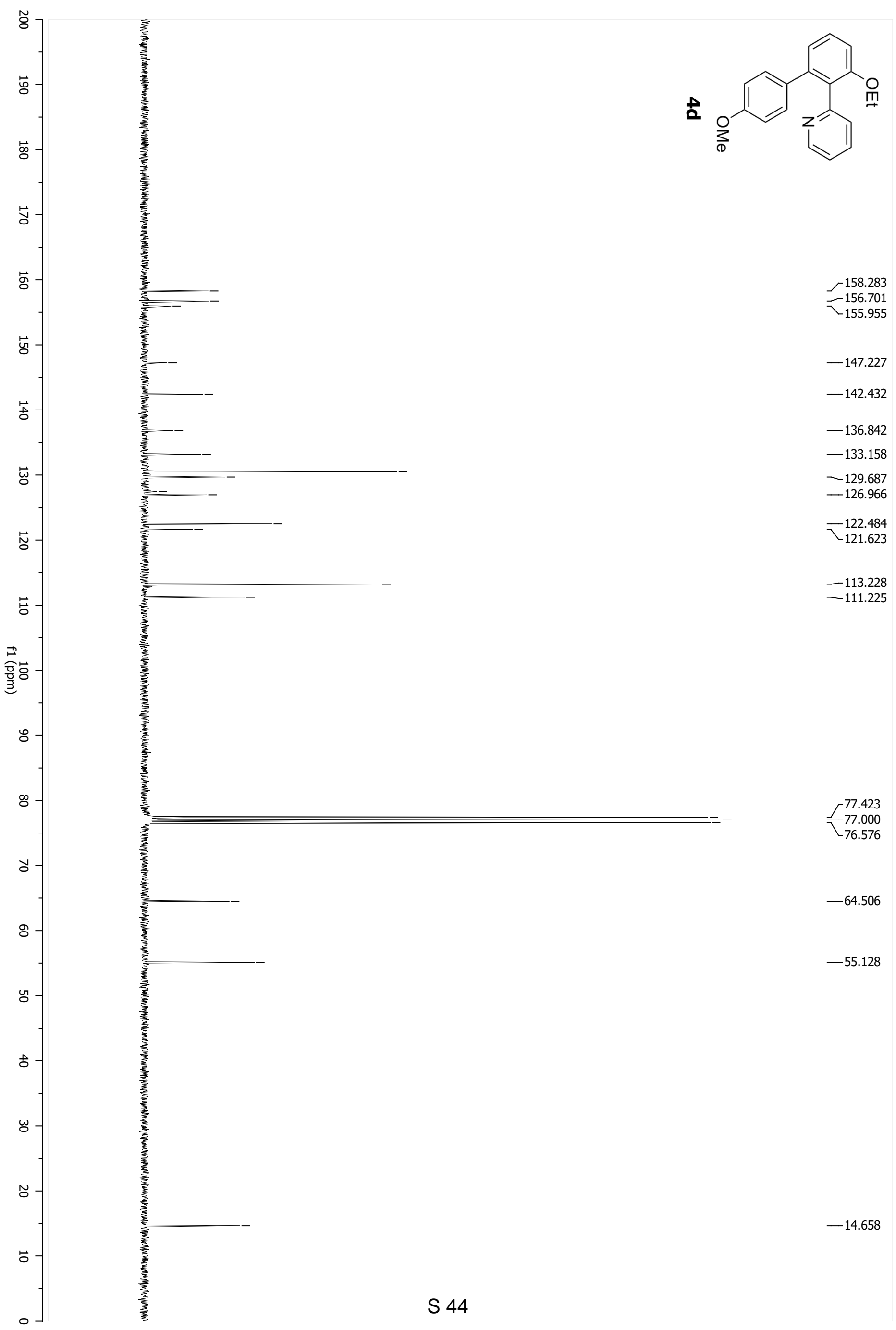




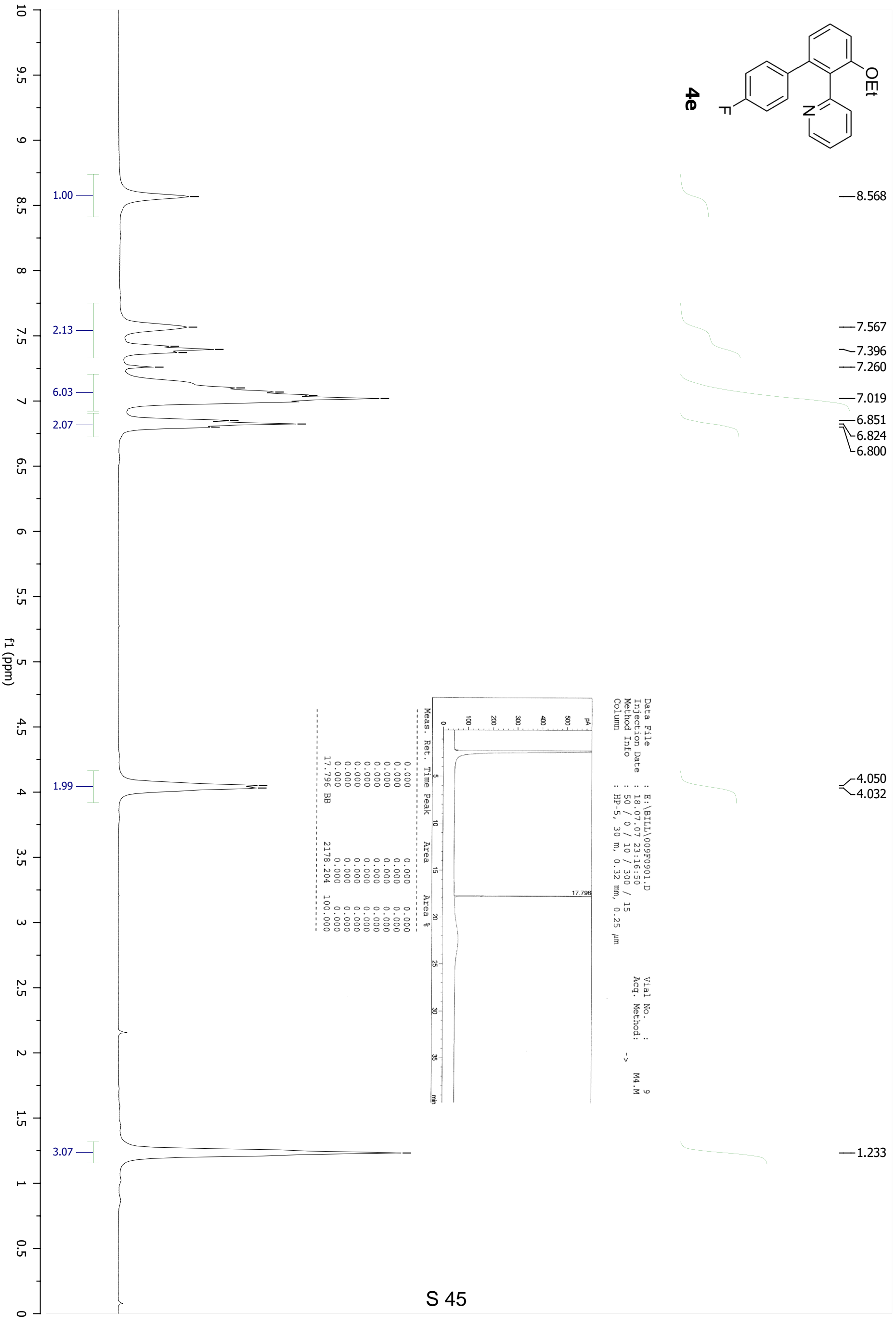




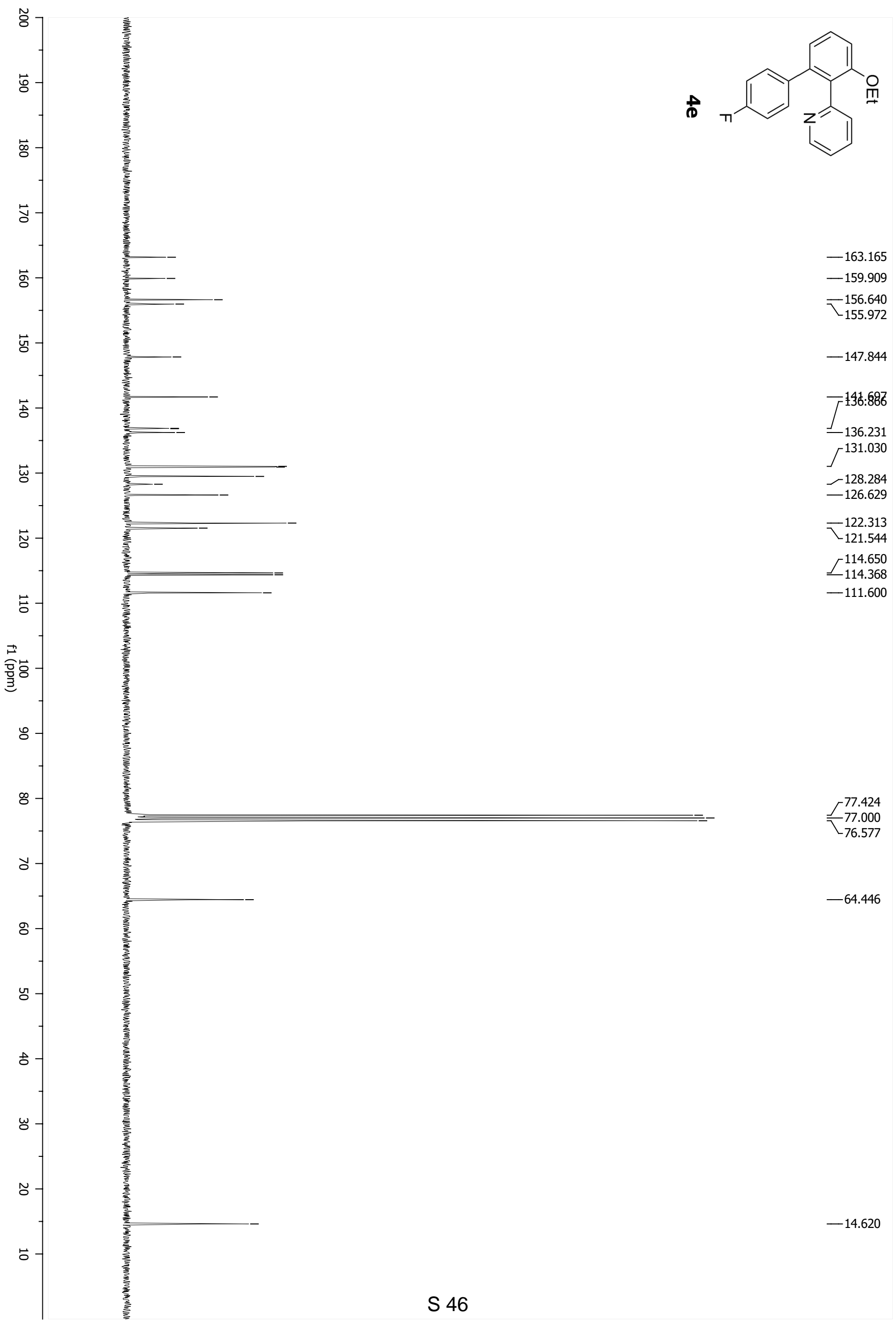




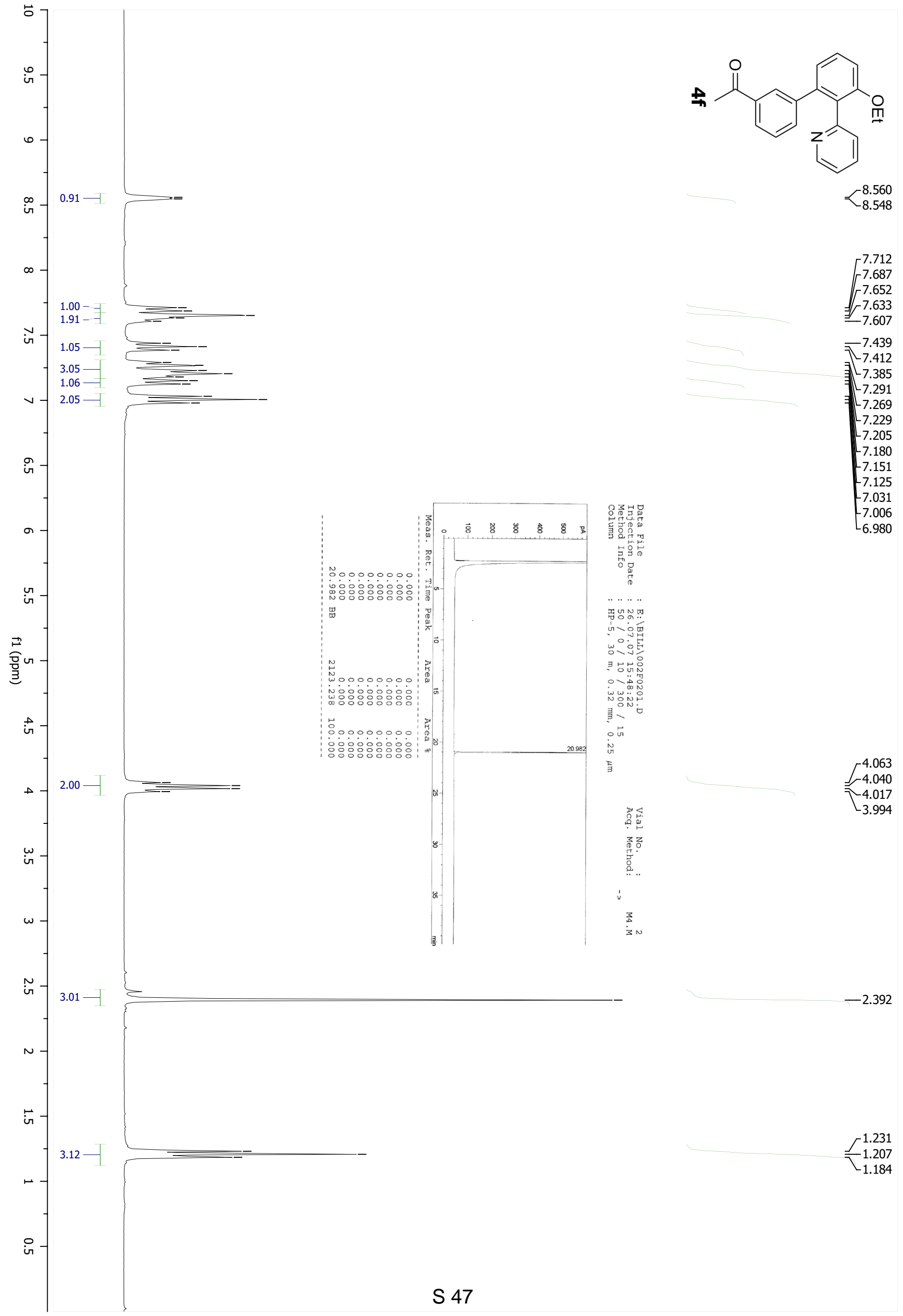




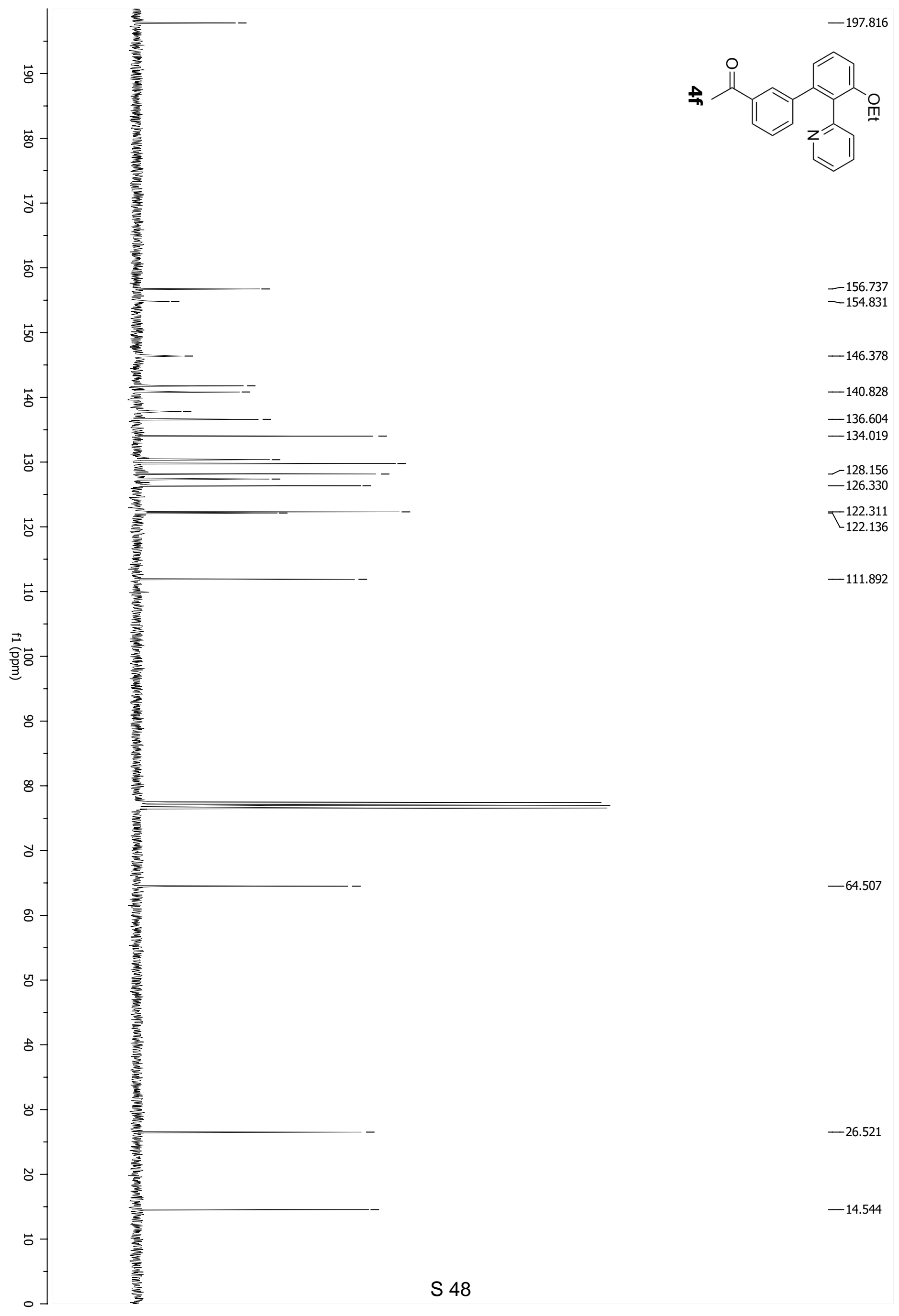




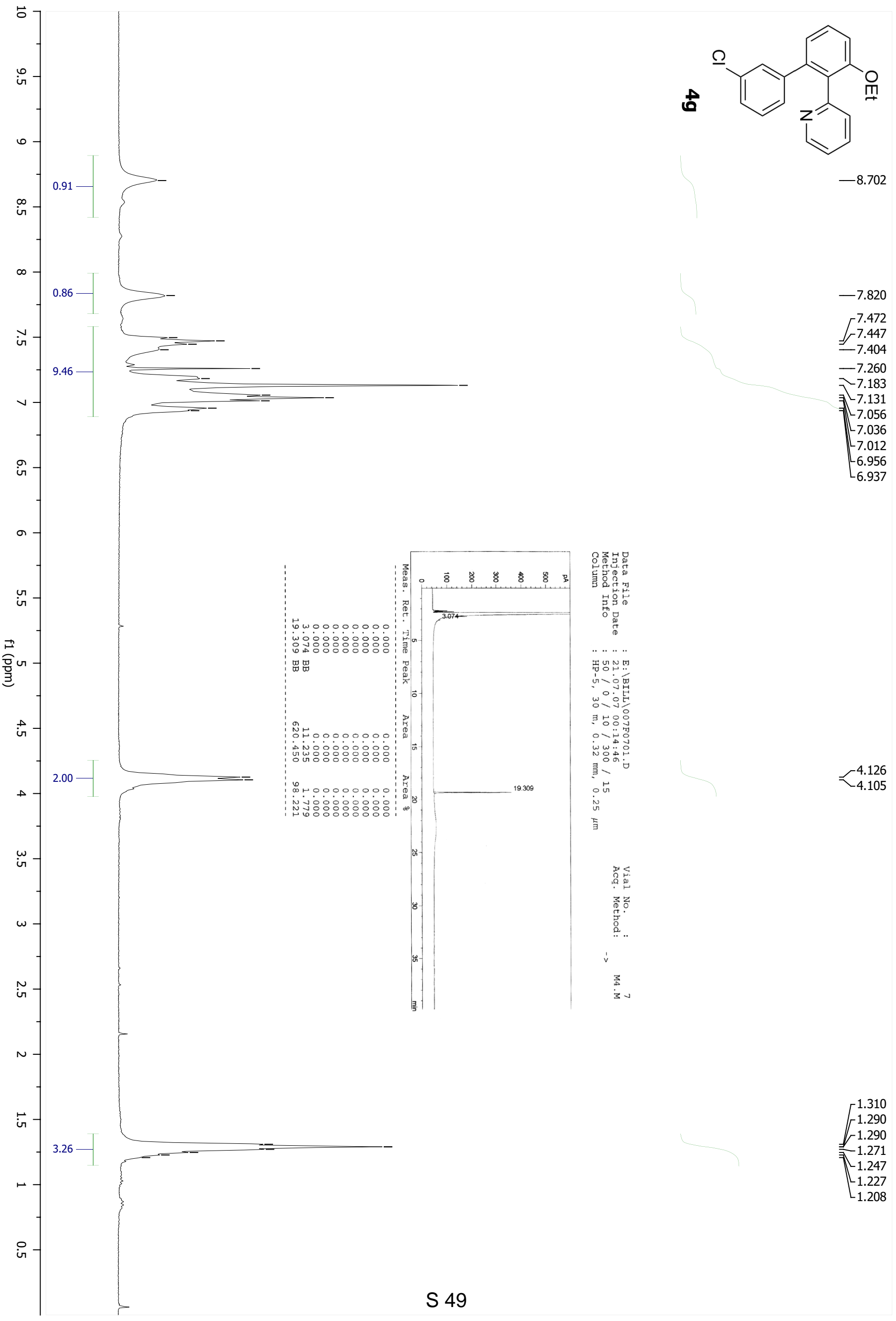




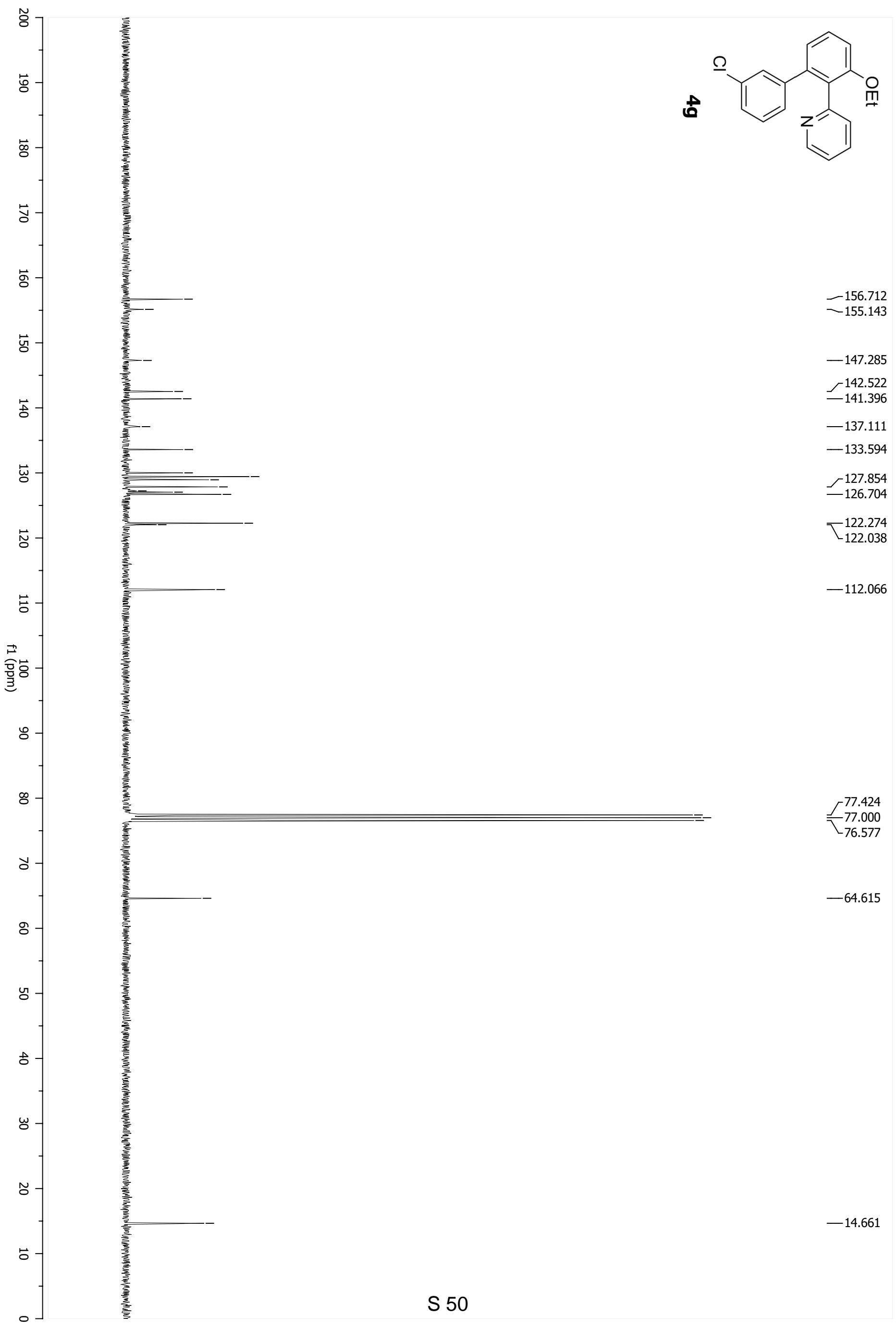




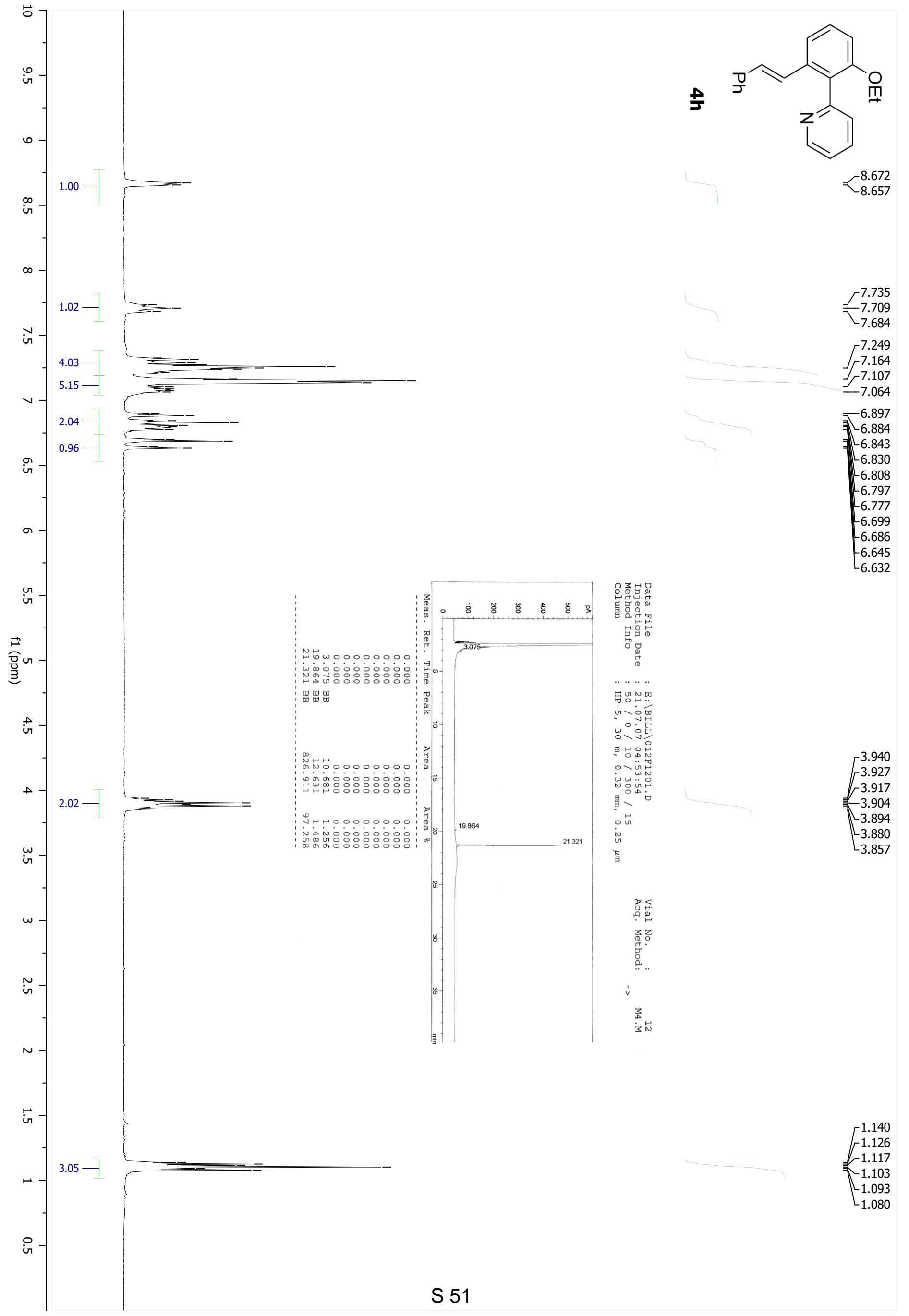




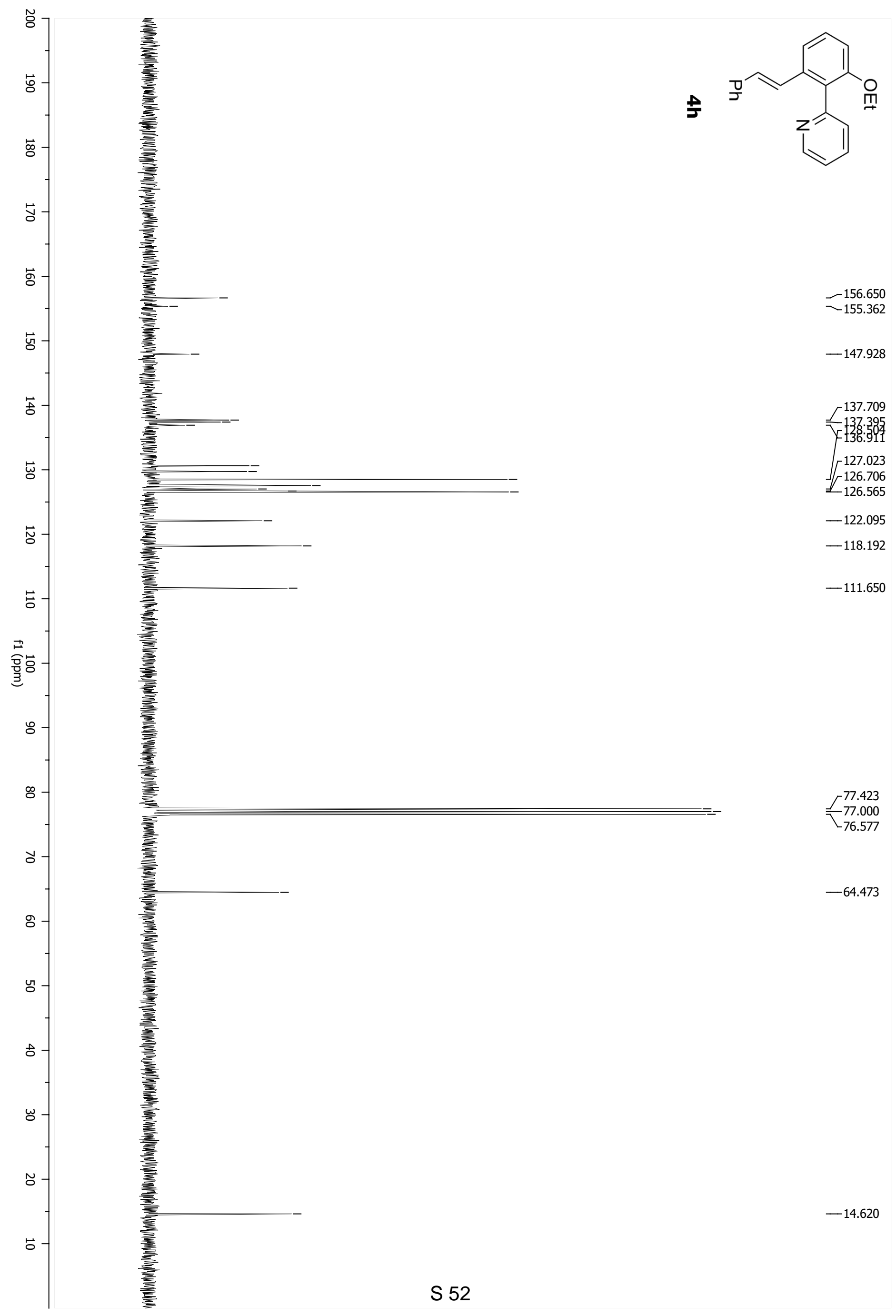




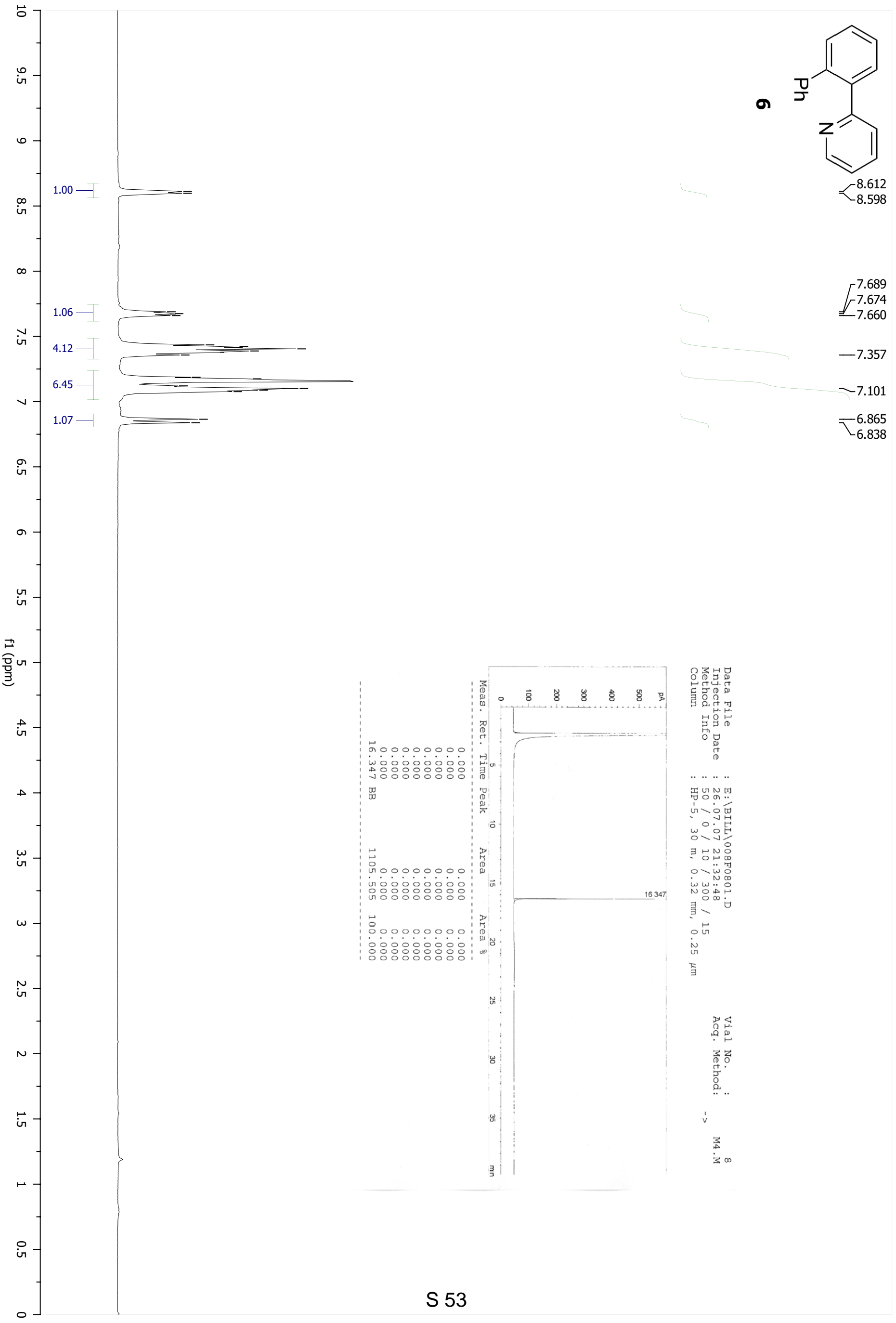




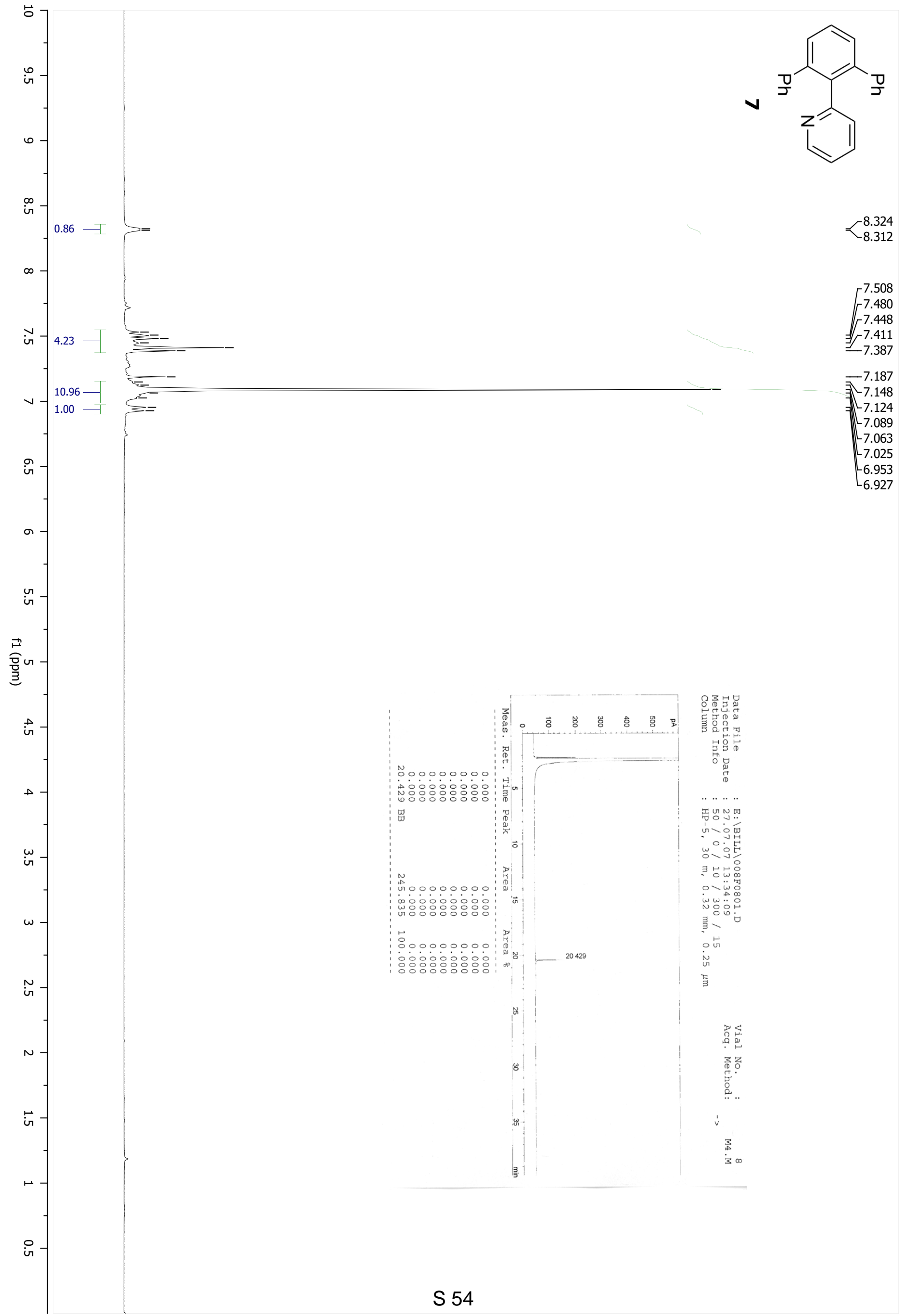




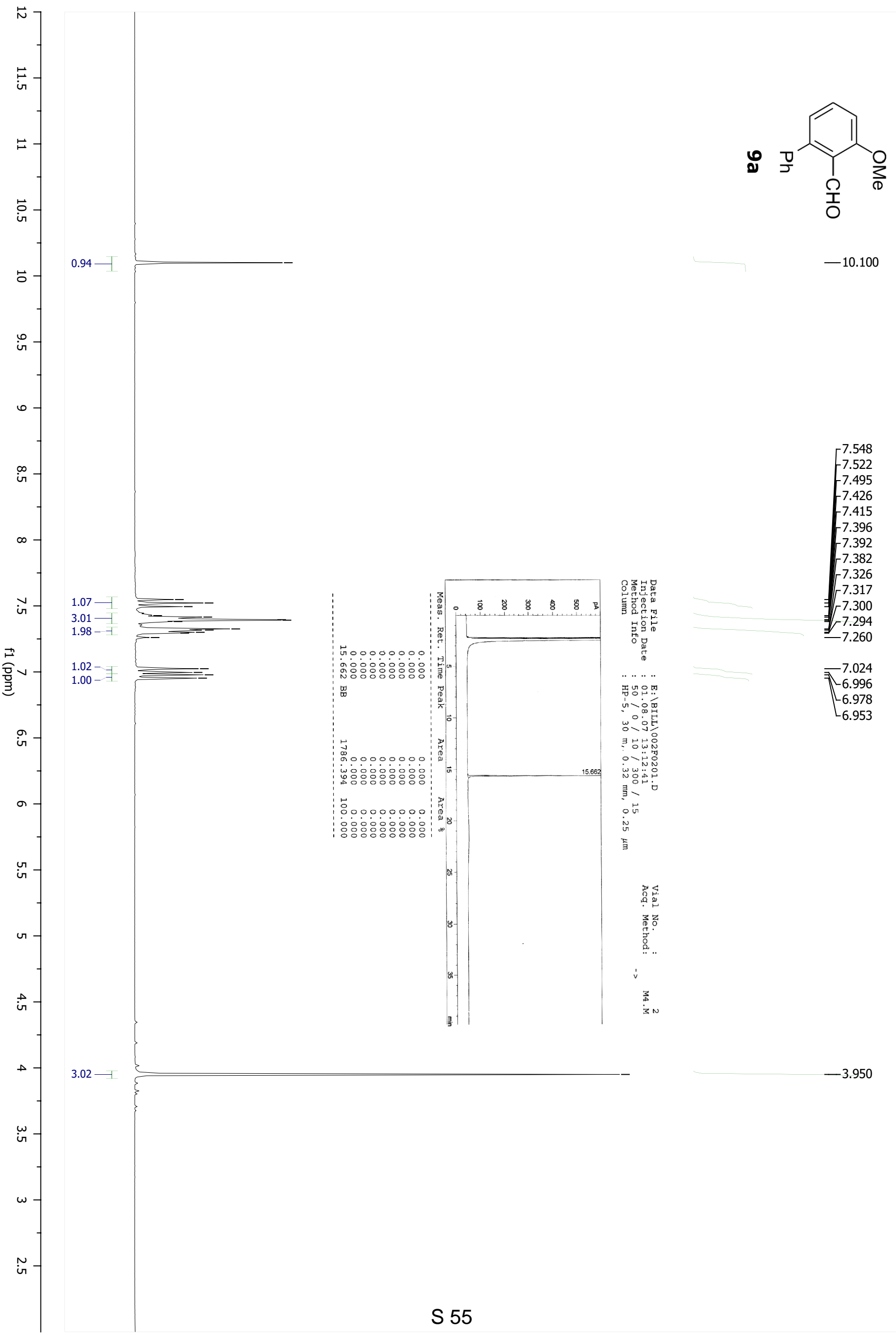




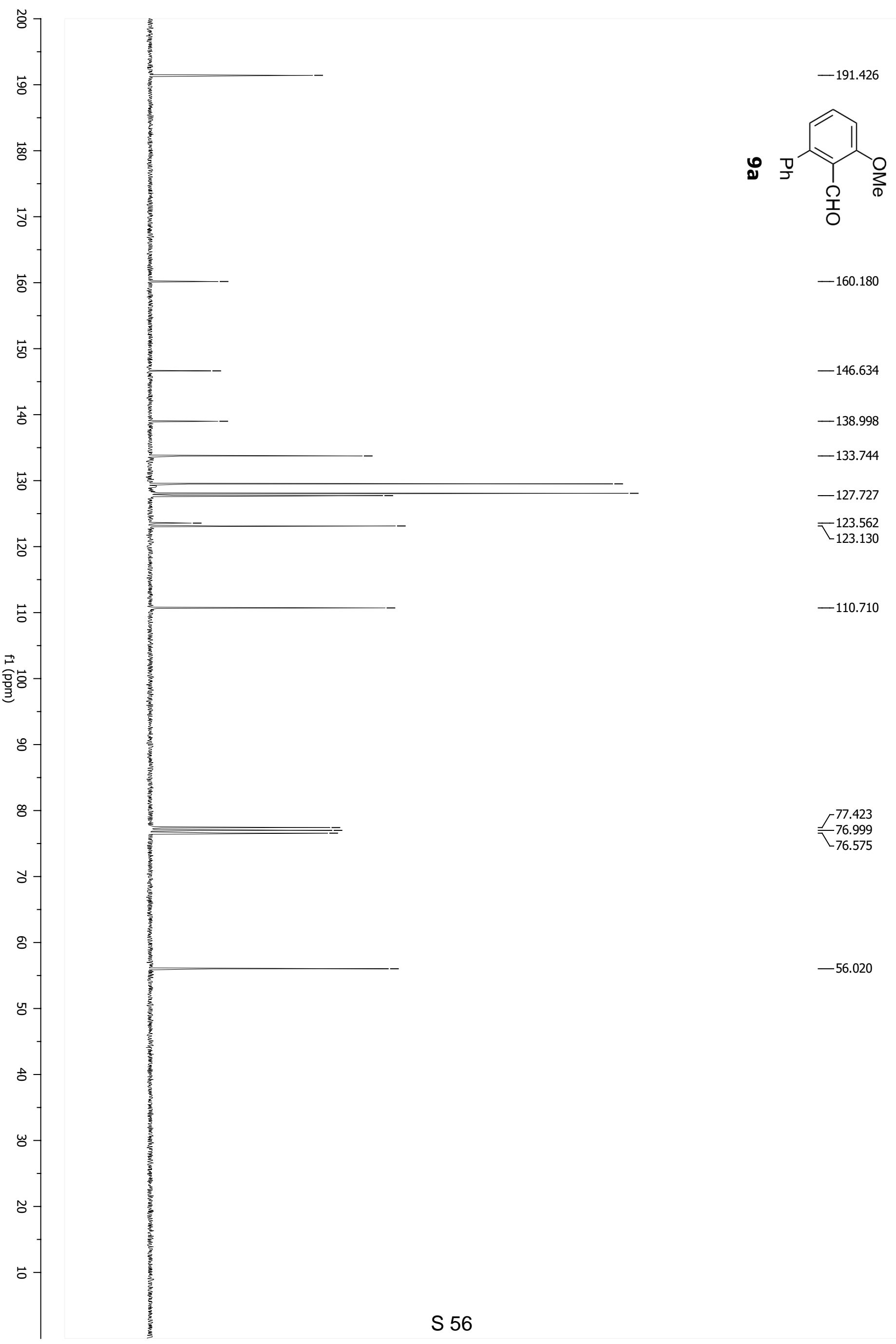




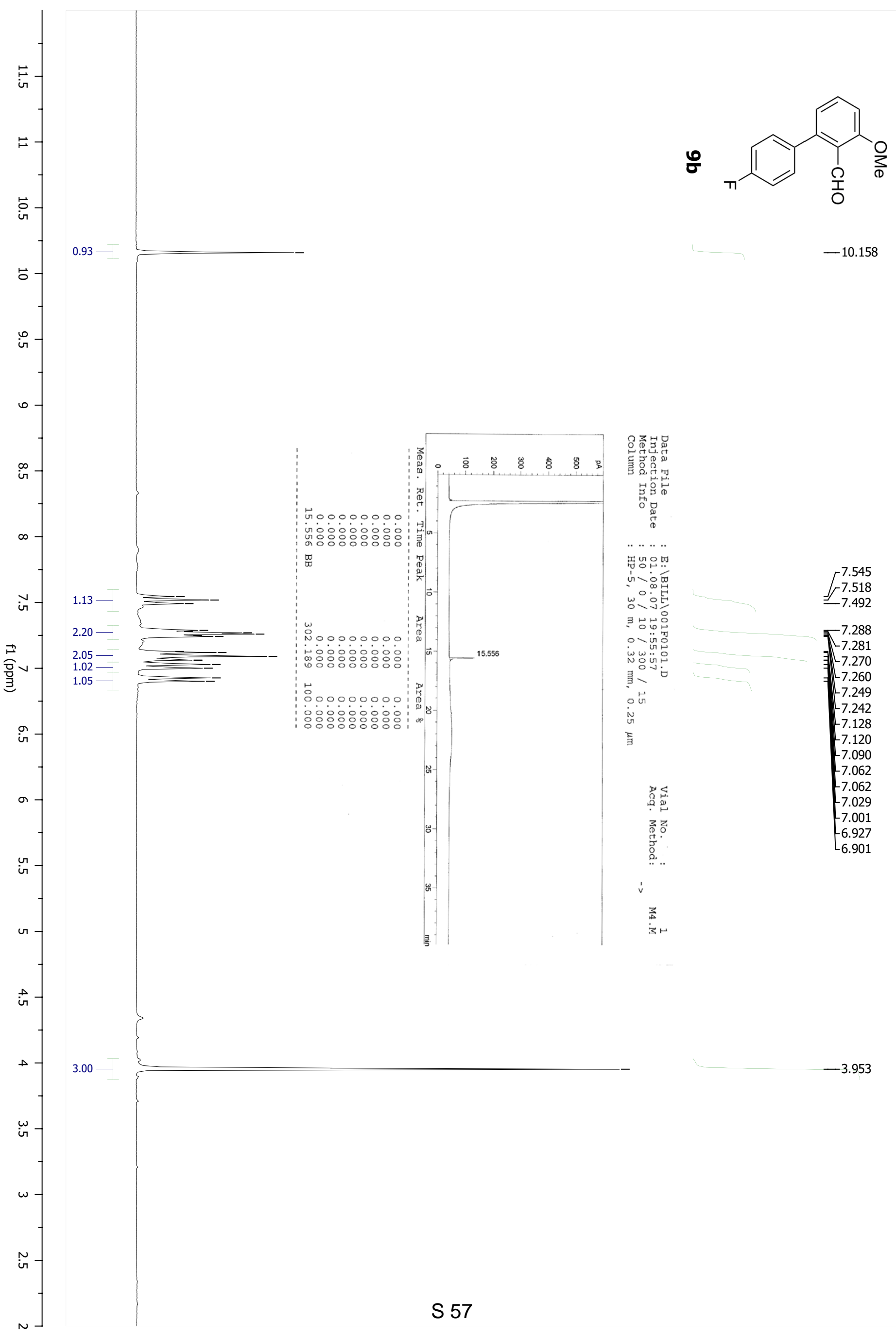




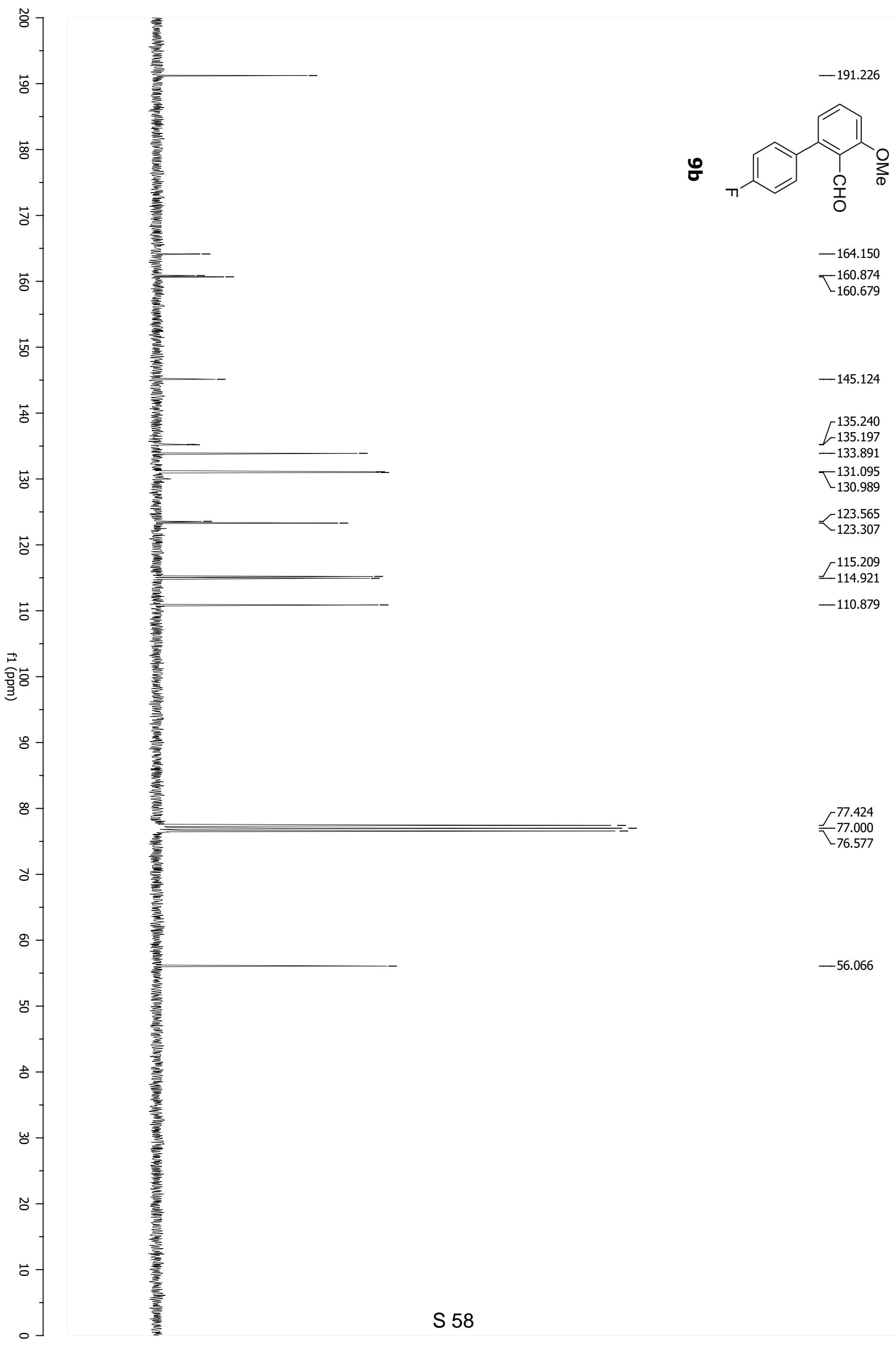

